\title{
Recent Developments in Cell Line Shipping Methods: Pivotal Gaps in Cellular Death Sciences
}

\author{
Shabnam Heydarzadeh ${ }^{1}$, Sima Kheradmand $\mathrm{Kia}^{2}$, Seti Boroomand ${ }^{3}$, and Mehdi Hedayati ${ }^{1}$ \\ ${ }^{1}$ Cellular and molecular endocrine research center \\ ${ }^{2}$ Laboratory for Red Blood Cell Diagnostics, Sanquin, Amsterdam, The Netherlands \\ ${ }^{3}$ Djavad Mowafaghian Centre for Brain Health, Faculty of Medicine, University of British \\ Columbia
}

January 9, 2023

\begin{abstract}
Contrary to remarkable advances within the cell therapy industry, scientists expose dissatisfied challenges associated with the preservation and post-thaw cell death globally. Post cryopreservation apoptosis is normally observed in cultures and scientists are focusing on incorporation of apoptosis inhibitors. Impressive transport of cells without affecting their survival and function is a crucial and pivotal factor in any practical cell-based therapies. Preservation of cells permits the transportation of cells between distances, as well as improvement of safety and quality control testing in clinical and research applications. The prosperity of transportation methods is evaluated through the viability and proliferation percentages of the transported cell. For many decades, the conventional methods of transferring cells globally having adverse effects and speculated to be a challenging and expensive method. The main purpose of some studies is the optimization of cell survival after cryopreservation. In the new generation of cryopreservation science, various experiments wish to discover suitable and alternative methods for cell transportation to ship viable cells at ambient temperature without dry ice or in media filled flasks. In this review we try to represent a summary of the detection of recent studies including dry preservation, hypothermic preservation, agarosegel based method, polymer based cryogel matrix, encapsulation method, fibrin microbeads, osmolyte solution composition, collagen-based scaffold, natural zwitterionic betaine, bio-inspired cryo-ink that have been performed alternative, effective and economic methods for shipping viable cells at ambient temperature.
\end{abstract}

Recent Developments in Cell Line Shipping Methods: Pivotal Gaps in Cellular Death Sciences Shabnam Heydarzadeh ${ }^{1}$, Sima Kheradmand Kia ${ }^{2}$, Seti Boroomand ${ }^{3}$, Mehdi Hedayati ${ }^{4}$ 


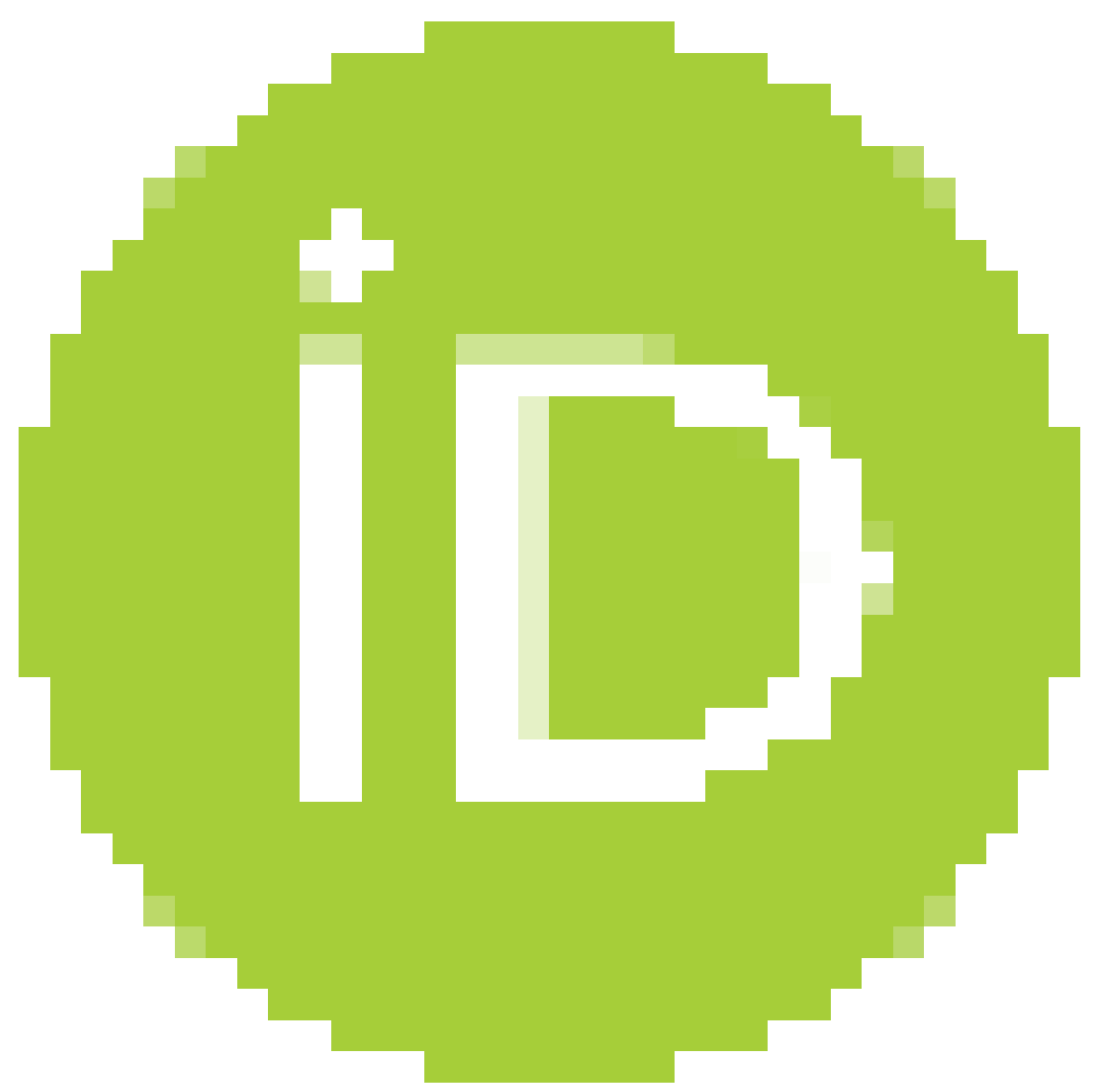

1. Department of Biochemistry, School of Biological Sciences, Falavarjan Branch Islamic Azad University, Isfahan, Iran, sh.heydarzadeh@endocrine.ac.ir,

https://orcid.org/0000-0002-9398-9211 


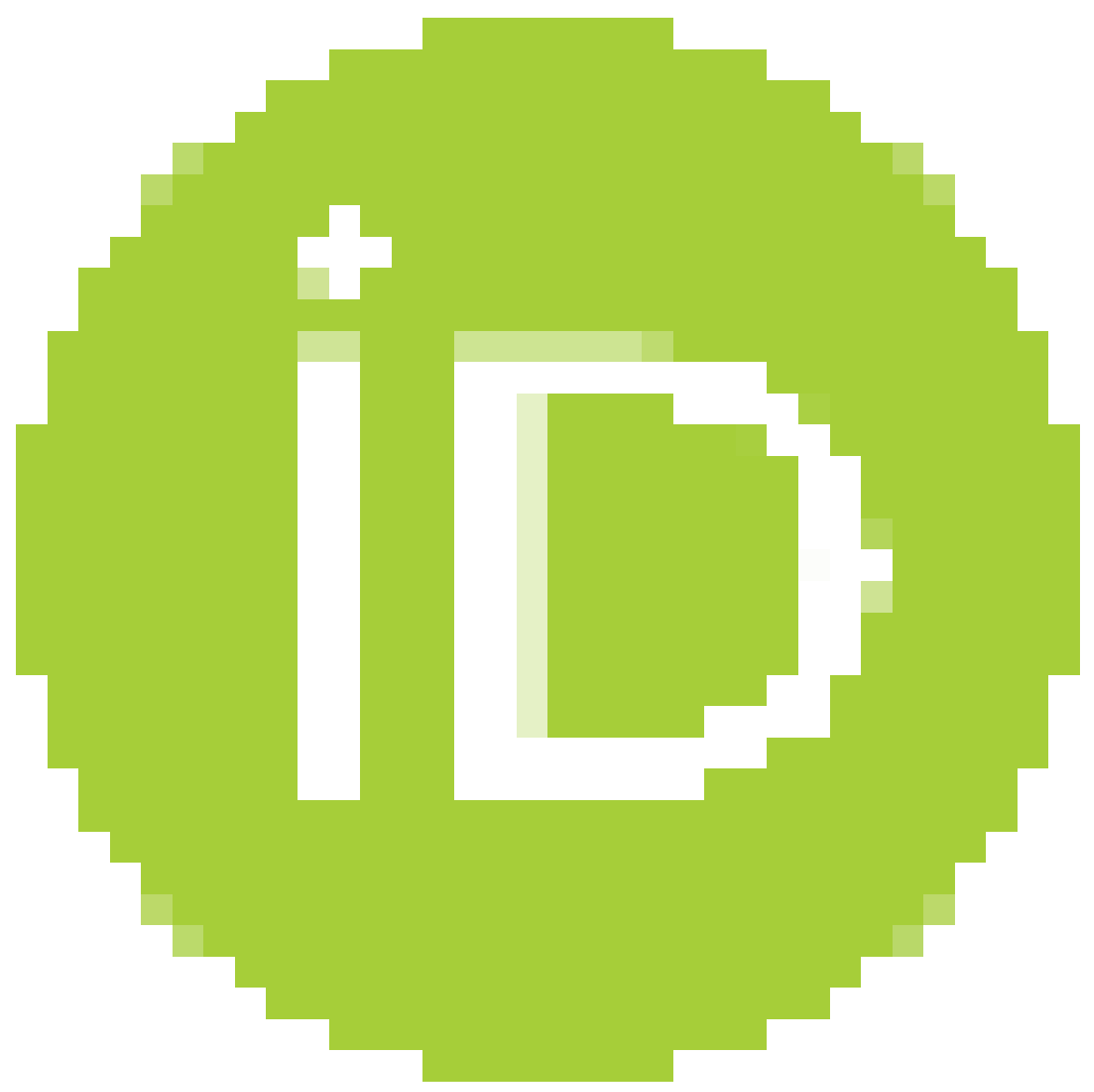

2. Laboratory for Red Blood Cell Diagnostics, Sanquin, Amsterdam, The Netherlands, s.kheradmandkia@sanquin.nl,

https://orcid.org/0000-0002-2693-1853 


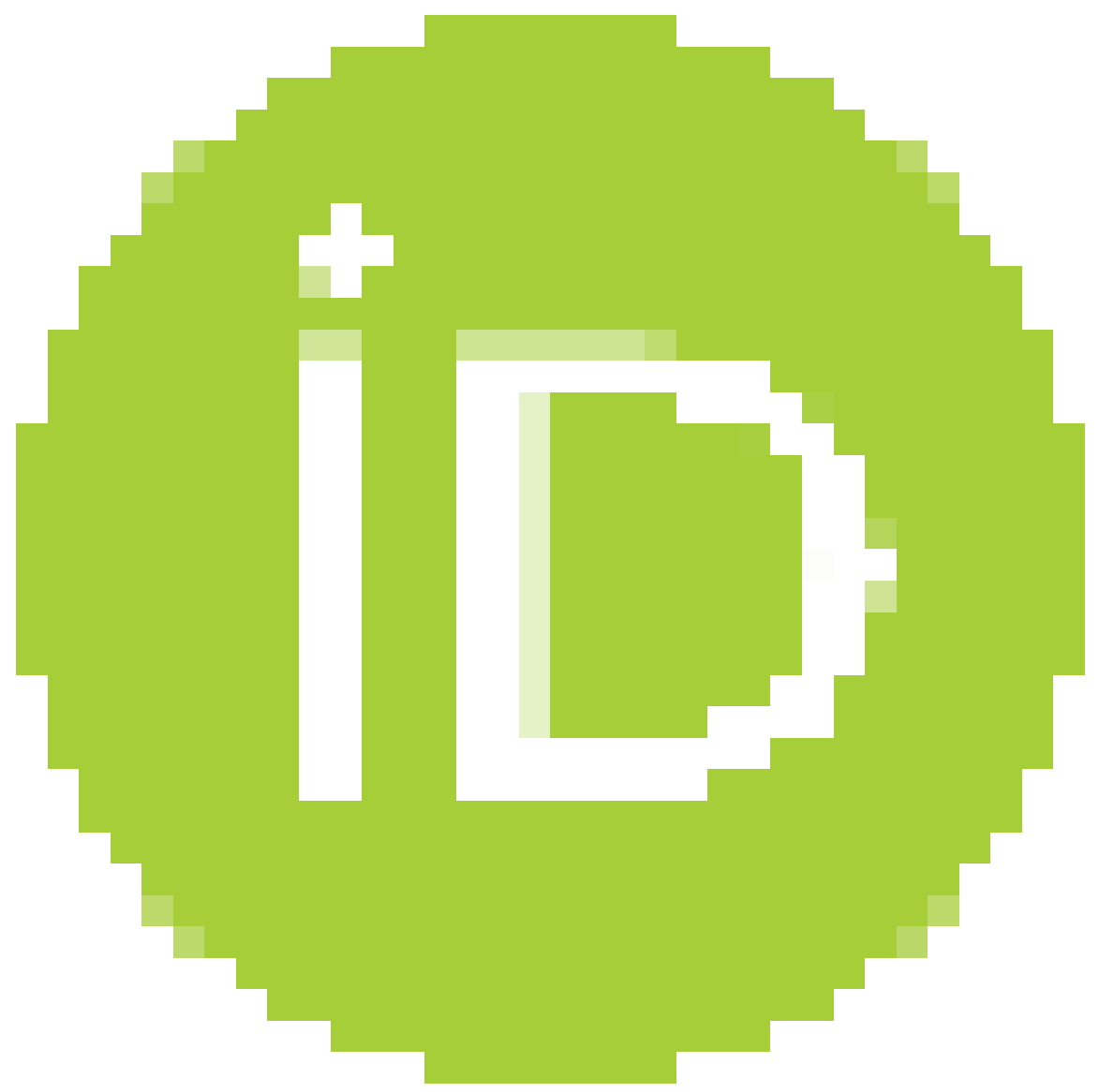

3. Djavad Mowafaghian Centre for Brain Health, Faculty of Medicine, University of British Columbia, Vancouver, British Columbia, Canada, Seti.boroomand@brain.ubc.ca,

https://orcid.org/0000-0002-6319-598X 


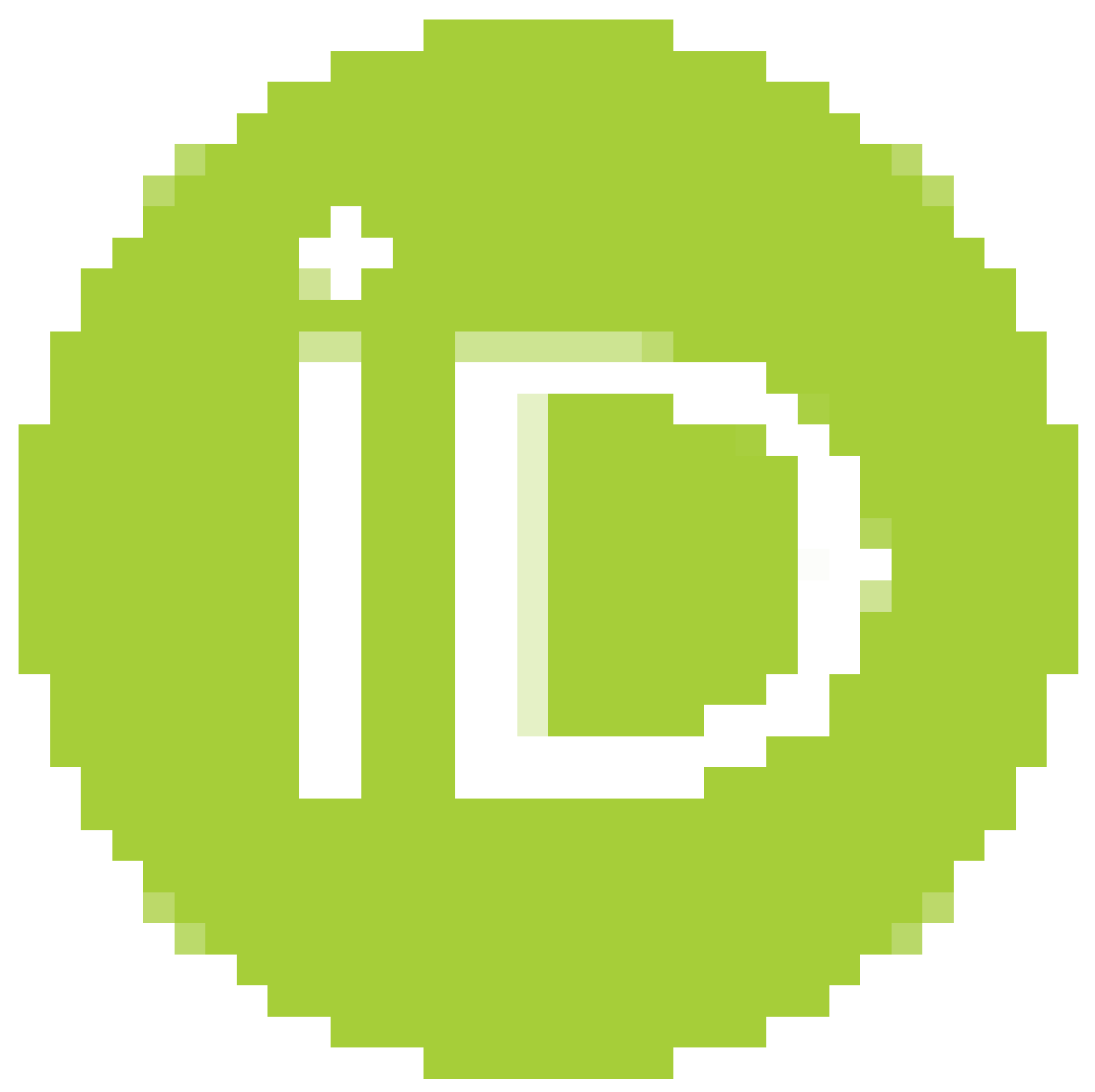

4. Cellular and Molecular Endocrine Research Center, Research Institute for Endocrine Sciences, Shahid Beheshti University of Medical Sciences, Tehran, Iran, hedayati@endocrine.ac.ir,

https://orcid.org/0000-0001-5816-775X

\section{*Corresponding Author:}

Mehdi Hedayati (Prof. in Biochemistry)

Cellular and Molecular Endocrine Research Center,

Research Institute for Endocrine Sciences,

Shahid Beheshti University of Medical Sciences, Tehran, Iran

E-mail: hedayati@endocrine.ac.ir \& hedayati47@gmail.com

\section{Abstract:}

Contrary to remarkable advances within the cell therapy industry, scientists expose dissatisfied challenges associated with the preservation and post-thaw cell death globally. Post cryopreservation apoptosis is normally observed in cultures and scientists are focusing on incorporation of apoptosis inhibitors. Impressive transport of cells without affecting their survival and function is a crucial and pivotal factor in any practical cell-based therapies. Preservation of cells permits the transportation of cells between distances, as well as improvement of safety and quality control testing in clinical and research applications. The prosperity of transportation methods is evaluated through the viability and proliferation percentages of the transported cell. For many decades, the conventional methods of transferring cells globally having adverse effects and speculated to be 
a challenging and expensive method. The main purpose of some studies is the optimization of cell survival after cryopreservation. In the new generation of cryopreservation science, various experiments wish to discover suitable and alternative methods for cell transportation to ship viable cells at ambient temperature without dry ice or in media filled flasks. In this review we try to represent a summary of the detection of recent studies including dry preservation, hypothermic preservation, agarose-gel based method, polymer based cryogel matrix, encapsulation method, fibrin microbeads, osmolyte solution composition, collagen-based scaffold, natural zwitterionic betaine, bio-inspired cryo-ink that have been performed alternative, effective and economic methods for shipping viable cells at ambient temperature.

\section{Keywords:}

Cell shipping, cell transport, cell death, cell culture, preservation, alternative methods, ambient temperature

\section{Running title:}

Cell Line Shipping Methods

\section{Graphical Abstract:}

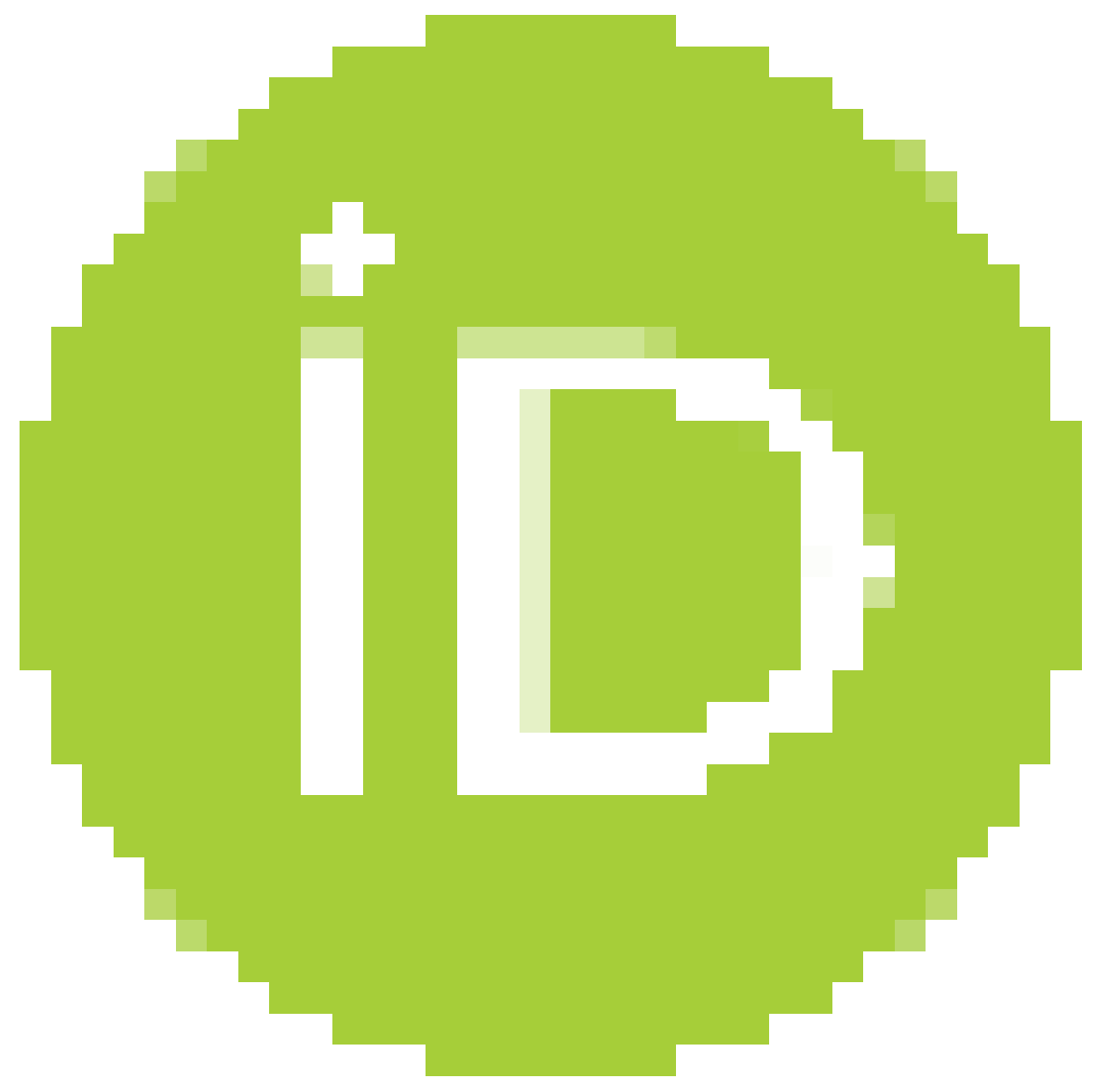

\section{Facts:}

- Shipping of cell lines across international borders is controversial and problematic issue.

- Contrary to remarkable advances within the cell therapy industry, scientists expose dissatisfied challenges associated with the preservation and post-thaw cell death globally.

- Representing alternative, effective and economic methods for shipping viable cells at ambient temperature is a vital requires of cell researches. 


\section{Open questions:}

- Why the development of alternative procedures for the global transfer and delivery of cells is an essential issue?

- Which cell shipping methods can be alternative for conventional methods of transferring cells with low post-thaw cell death and cytotoxicity?

- When can promise to prosperity of cell transportation methods?

- What is the main problems of cell shipping process and how can overcome?

\section{Introduction:}

The influence of cell culture technology on human society is indescribable. One main aspect of cell culture is the transportation and storage of cells in which special conditions should be implemented for the maintenance of cells for this prolonged time interval. Therefore, shipping of cell lines across international borders can be controversial and problematic. Recently, evolutions and improvements in biology science, efficacy of new drugs, manufacture of vaccines and biopharmaceutics and reproductive technology has depended seriously on cell culture technology. The quality of the medium and cell culture possible problems directly influence the research results and support cell survival and proliferation and cellular functions. Cell culture possible problems including cross-contamination, bacteria, fungi and mycoplasma contamination, chemical contamination are the most complex challenges in tissue culture (Cobo et al., 2005; Nema \& Khare, 2012; Yao \& Asayama, 2017). Cell-based therapies involve a time lag between the preparations of tissue regeneration and

implantation. Special conditions such as optimal $\mathrm{CO} 2$ rate and temperature should be implemented for the maintenance of cells for this prolonged time. It is obvious that while cells in suspension are being transferred in ambient conditions they may not survive for long time intervals (Reddig \& Juliano, 2005). Therefore, the development of alternative procedures for the transfer and delivery of cells is important (Ehrenreich \& Ruszczak, 2006; Schoenhard \& Hatzopoulos, 2010). Although these procedures are complicated and expensive and involve special cell culturing conditions, but developing simple methods with minimal manipulation and maximal survival is preferable and more relevant for clinical applications. In spite of the importance of this issue, scarcely has focused on reports on the prevention of cell death in suboptimal conditions when cells are transported to the clinic (Gorodetsky et al., 2011; Suuronen et al., 2008; Theus et al., 2008).

One main aspect of cell culture is the transportation and storage of cells. Cells are often transported from one city to another and also from one country to another. Thus, convenient and low-cost methods are desirable for routine transportation of cells at long distance. There are many existing methods for cell transportation in cell culture based research. The prosperity of these methods is evaluated through the viability and proliferation percentages of the transported cell (Ebertz \& McGann, 2004; Esfandiarei et al., 2007; Kumari \& Kumar, 2017). Impressive transport of cells without affecting their survival and function is a major factor in any practical cell-based therapy. For many decades, the most common method of transferring cells between institutes and companies is shipping cryopreserved cells on dry ice usually treated with anti-freeze solutions; most commonly DMSO because it is less toxic than many alternatives (B. Chen et al., 2013; Hanna \& Hubel, 2009). The cost of process from storage to delivery is high. In addition transit delay is repeatedly observed so by evaporation of the dry-ice, cells bathed in cryoprotectant DMSO, which is cytotoxic at ambient temperatures. Cell culture facilities will most likely continue to use this mode of shipping for the advantage of eliminating large $\mathrm{CO} 2$ emission associated with using dry-ice. So lowcost, simple, inexpensive and non-hazardous methods of transporting cell cultures are desirable for routine transportation of mammalian cells at long distances (Stevens et al., 2007).

The commonly used technique for cell transportation is the shipping of cryopreserved cells on dry ice. Transportation of cells in different countries by this method is limited. According to Federal Express shipping information, more than $50 \%$ of countries around the world forbid delivery with dry ice because of hazardous nature and for its possible cause of explosion and suffocation. Liquid nitrogen vapor shippers are usually designed for the safe transportation of valuable cells and biological agents because it is cost effective (Mantel et al., 1979; Junjian Wang et al., 2015). Cryopreservation is based on slowing the vital cellular functions. Formation of intra- and extracellular ice crystals can cause destruction of cell membranes and cell organelles 
which leads to cell damage (D. Gao \& Critser, 2000; P Mazur, 2004). On the other hand, cell dehydration due to osmotic results leads to denaturation of proteins and complete destruction of the cell (Grein et al., 2010; Karlsson \& Toner, 1996). For these reasons, many researchers have focused on the optimization of cell survival after cryopreservation by investigating the influence of different cooling and thawing rates for certain cell types (Armitage, 1987; Meryman, 2007). Besides the optimization of cooling and thawing rates, studies focus on the investigation of cryoprotectants. The main properties of these chemicals for protection of cells from cellular damage during cryopreservation are the stabilization of the cell membrane (Rubinstein et al., 1995; Rudolph \& Crowe, 1985) and/or the decreasing of osmotic effects (Baust et al., 2003).

Some investigators simply send cell culture flasks filled with medium, but actually agitation can cause cell monolayers to detach. Liquids pose a spill hazard, and are forbidden in the mail and air-travel (Stefansson et al., 2017; Wheatley \& Wheatley, 2019). This also has limited shipping time problem that cells in media have limited viability with flask leakage. It is just suitable for transporting cells across a short distance and over a very short period of time (usually up to 24 hours). Several types of cells do not survive and grow well through transportation in cell culture flasks due to rapid exhaustion of oxygen and $\mathrm{pH}$ change (Junjian Wang et al., 2015). However, using liquid nitrogen for the transfer of stem cells requires a short delivery time window, is technically challenging and financially expensive (B. Chen et al., 2013). Shipping cells in liquid nitrogen is hazardous and can be prohibitively expensive. The more economical and less dangerous alternative is to ship frozen cells using dry ice (Stefansson et al., 2017). Recently, several studies intended to find suitable materials and methods for transporting cells without dry ice (Junjian Wang et al., 2015). Novel alternative procedures for transportation of viable cells at ambient temperature without dry ice or in media filled flasks have been investigated in recent experiments.

From a development perspective, a truly "ready to go" cells system for high throughput application is now possible. By these procedures scientists will have functional cells already plate to perform assays. Ambient temperature shipping is a modern method to transfer cells. Instead of sending cells in dried ice using a Styrofoam box, cells can be shipped in a standard box at room temperature. Another key aspect is the environmental and regulation aspect that must be taken into consideration. Styrofoam is non-ecofriendly and shipping in dry ice is disallowed in many locations (Stefansson et al., 2017). At atmospheric pressure, liquid nitrogen boils at -196@C, while sublimation of dry ice occurs at -78.5@C. Although cells can be kept at -78.5@C for a period, but decreasing of viability, loss of dry ice and thawing of the sample in transit will kill the cells because of DMSO toxicity (Vigneault et al., 2009). The ability of preserving cells is serious for their application in clinical and research studies. With the various diversity of cells that require to be preserved, attentive optimization of preservation protocols seems tedious and shows significant restriction (B. Chen et al., 2013; Heo et al., 2015; A. P. Li, 2007; Linnebacher et al., 2010). Preservation of cells allows the transportation between sites, completion of safety and quality control examinations. For the reason of great diversity of cells which need to be preserved, accurate optimization of preservation protocols seems tiresome and indicates remarkable limitations. In this review, we will focus on summarizing the findings of recent preservation studies that have been performed.

\section{Search strategy:}

A systematic literature search was performed using PubMed, Web of Science, Google Scholar, Scopus, and ScienceDirect as an international database from 1978 up to 2020 with English language restriction. The search key words were: Cell shipping, cell transport, cell culture, preservation. All English language and free full text paper selected.

\section{Cryopreservation methods:}

Under in vitro condition one of the vital environmental factors for cell survival is temperature. A standard condition for mammalian cells is to culture usually in the incubator which in CO2 levels are set to 5\% and temperature to 37@C unless specific research purpose is required (Brown, 1990). According to previous studies low temperature can decrease cell growth rate (Azambuja et al., 1998; Kaushik \& Kaur, 2005; Vergara et al., 2014); whereas mild heat stress enhances cell proliferation rate and accelerates development 
(Choudhery et al., 2015; Tounkara et al., 2012). Increasingly, long-term treatment at sub-zero temperature can intensely influenced the viability rate of mammalian cells (Jin et al., 2008; Moussa et al., 2008; Juan Wang et al., 2017). Utilization of cryogenic temperatures are the most known methods for the long-term preservation of live cells. Attentive optimization of these protocols is most often inescapable for achieving high yield and survival of specific cells (Heo et al., 2015). Cryopreservation is classified into slow freezing and vitrification methods (Table 1). The main dissimilarity between these two methods are the concentrations of cryoprotective agents (CPAs) and the cooling rates used (D. Gao \& Critser, 2000; Jang et al., 2017).

A common method for cell shipping is that cryopreserved cells are transported with dry ice with a foam container; which have little influence on cell features and maintains a high rate of cell viability (Prent $\varnothing$, 1997). However, cell shipment with dry ice is costly and forbidden by the aviation departments of many countries (Junjian Wang et al., 2015). Cryopreservation is a procedure that preserves biological samples by cooling them in very low temperatures. Cellular events such as ice crystal formation, osmotic shock and membrane damage are the main reasons that cells cannot be stored with ordinary freezing methods for a long time and it will lead to apoptosis. In recent years, the prosperous cryopreservation of cells and tissues has been gently increasing with the extension of cryoprotective agents and temperature control instruments. This review, outlines and clarifies cryopreservation processes, including slow freezing and vitrification (Jang et al., 2017).

According to the cell types, there is substantial variety in cryosurvival response through the freezing and thawing cycle. The important stages in cryopreservation are: the mixture of cryoprotectant with cells or tissues, cooling and storage, warming, removal of CPAs after thawing. The proper utilization of CPAs is therefore influential to improve the viability of the sample to be cryopreserved (H.-H. Gao et al., 2016). Cellular mechanical injury in fast freezing is associated with intracellular ice formation in water to ice phase transition. Whereas slow freezing causes osmotic rupture due to exposure to extra- or intracellular high concentrated solutions and intracellular ice formation. Freezing is lethal for most living cells however cryopreservation retains biological samples at cryogenic temperature to preserve the fine structure of cells. The freezing manner of the cells can be different in the presence of a cryoprotective agent, which influences the rates of water transport, nucleation, and ice crystal growth (Peter Mazur, 1970; Sambu, 2015).

\section{Slow freezing}

In slow cooling conditions, cells could efflux intracellular water quickly enough to stop intracellular ice formation (Schoenhard \& Hatzopoulos, 2010). Optimal cooling rates are related to cell types because there are some differences in the capacity of different cells to move water across the plasma membrane. Slow freezing can lessen cell injury and maintain the permeability of the cell membrane through substitution of the cytoplasm water with CPAs (Mandawala et al., 2016; Yong et al., 2015). Freezing for long-term storage has proven to be one of the most effective methods of maintaining a stable supply of various cell types. However, cells may be damaged by environmental changes during the freezing process (Miyamoto et al., 2018). There are various factors that influence the function of cells cultured after cryopreservation and thawing, including the cryopreservation solution (Miyamoto et al., 2006; Miyamoto, Noguchi, et al., 2012; Miyamoto, Oishi, et al., 2012), biomaterials (Miyamoto et al., 2009), freezing methods (Fujioka et al., 2004), and freezing and preservation temperatures (Reubinoff et al., 2001). Among cryopreservation solutions, cryoprotective agents such as glycerol, ethylene glycol, and dimethyl sulfoxide (DMSO) are the most effective due to their high rate of penetration into cells. In addition, it has been reported that starch and oligosaccharides like trehalose and maltose are effective in suppressing damage to cells (Y. Chen et al., 1993; Crowe \& Crowe, 2000; Miyamoto et al., 2018).

Cryopreservation with controlled slow cooling rate was first exposed to discussion by Polge, Smith and Parkes in 1949 using the protective properties of glycerol (GLY) (Karlsson \& Toner, 1996). Dimethyl sulfoxide (DMSO) was known as a cryoprotectant in 1960 by Lovelock and Bishop which was considered to have more widespread applicability than glycerol in tissue culture (Fahy et al., 2004). The development of a cryopreservation protocol for a given cell type requires specification of: pre-freeze processing; introduction of a cryopreservation solution; freezing protocol; storage conditions; thawing conditions and post thaw assessment 
(Hanna \& Hubel, 2009). During cryopreservation, cells are cooled slowly in the presence of a cryoprotectant to reduce the formation of ice crystals. Because different cell types have unique biophysical and biological characteristics (Stacey \& Masters, 2008). During cryopreservation, slower than optimal freezing rates can cause cell damage by the formation of intracellular ice and have deleterious effects on cell viability, while concentrations of cryoprotectant that are too high could be toxic to the cells. To prevent this, an ideal cooling rate and cryoprotective agent (CPA) should be chosen (Heo et al., 2015).

CPAs are classified into intracellular and extracellular agents. The intracellular one penetrates inside the cells and prohibits ice crystal buildup and membrane rupture. The extracellular compounds do not penetrate the cell membrane and decrease the hyperosmotic effect through the freezing duration. Among CPAs, DMSO, propylene glycol (PG), ethylene glycol (EG) and glycerol (GLY) are intracellular compounds. Extracellular CPAs are known as trehalose, sucrose, dextrose, and polyvinylpyrrolidone. DMSO is the frequently utilized CPA. It provides a high rate of cell survival during the freezing time but causes chemical cytotoxicity at room temperature which can lead to cell injury osmotically. This cytotoxicity obligates the researchers to perform the experiment quickly. Moreover, different experimenters need diverse amounts of time to thaw their cells completely and to wash them free of CPA. Consequently, less cytotoxic CPAs are required for the standardization of this course of actions (Janz et al., 2012; Syme et al., 2004; von Bomhard et al., 2016). An alternative method extensively used by local companies or laboratories is directly to ship the cultured cells in the flask fully filled with cell culture medium; but the disutility of this method is to not be appropriate for long-distance shipment (Juan Wang et al., 2017).

\section{Vitrification}

It is now believed that with current methods of cryopreservation, unrestricted ice formation in cells and tissues at subzero temperatures is the most vital factor that is seriously important in survival procedures during freezing and thawing. Recently, this important problem has been efficaciously circumvented in some tissues by using ice-free cryopreservation techniques based on vitrification which is a fast freezing method that has become increasingly popular (Taylor et al., 2019). This hopeful and simple process keeps away cells from injuries caused by their dehydration during slow freezing. To prevent intracellular ice buildup, a higher concentration of CPA is necessary, but its cytotoxic effect can be reduced by immediate freezing in liquid nitrogen (LN2). The study of evaluation of comparison between vitrification and controlled slow freezing methods under standardized conditions has been performed recently (Table 1.). According to scientific reports, the cell toxicity depends on the chemical properties of CPAs and cell types. The investigation around the effect of various CPAs and their combinations on cell types cannot be predicted well, and hence it dependes on more experimental approaches (Fan et al., 2009).

Vitrification known as ice-free cryopreservation is an alternative preservation for slow-freezing technique which uses very high concentration of cryoprotectant cocktails to prevent ice formation by directly transforming from the aqueous phase to a glass state after direct exposure to liquid nitrogen (Fuller et al., 2016). Cryoprotectant concentrations as high as 4-8 $\mathrm{M}$ are typically required. Because such concentrations are very toxic for most cell types, individual protocols are optimized for each cell type depending on its sensitivity to the cryoprotectant (Heo et al., 2015). Vitrification significantly relies on three main factors including: viscosity of the sample; cooling and warming rates; and sample volume. A fine harmony must be maintained among all the related factors to certify a successful vitrification. There are two methods of vitrification that are classified into individual two methods: equilibrium and none quilibrium. Equilibrium vitrification needs formulation of multimolar CPA mixtures and their injection into the cell suspensions. Nonequilibrium vitrification is divided into carrier-based systems and carrier-free systems. A major advantage of vitrification is the low risk of freeze injury, therefore warrant a sufficiently high cell survival rate. It has reported that the probability of contamination with pathogenic agents is high so the technique requires good manipulation proficiency (Yavin \& Arav, 2007).

Alternative procedures for vitrification protocol optimization have centralized on enhancing the cooling rate, which could permit lower concentrations of cryoprotectant for glassy-state achievement. Technologies to allow faster cooling rates have been described in which cells are placed inside thin open straws, cryotops, 
electron microscopy grids, or cryoloops. More recently, scientists validated the use of quartz micro-capillaries for vitrification, which enabled ultrahigh cooling rates and required significantly lower concentrations of cryoprotectants than standard vitrification protocols ( 2.0 M) (Criado et al., 2011; He et al., 2008; Kuwayama et al., 2005; H.-J. Lee et al., 2009; Vajta et al., 1998). To dominate the restriction of current cryopreservation and vitrification protocols, a vitrification protocol has developed that can be easily standardized and performed without changes for a wide variety of cell types. To improve previous quartz capillary technique, fused silica microcapillaries have been employed, which are industrially manufactured to have well controlled dimensions and physical properties. Compared with most current cell vitrification technologies, this method contains fused silica microcapillaries performed like a low-thermal-mass, high-heat conductivity cell container to raise the cooling rate by an order of magnitude. The vitrification process is simple and can be done in less than 15 minutes, significantly faster than conventional vitrification techniques (Heo et al., 2015).

Vitrification is a high warming and cooling rate method. Cryopreservation strategies focus on the use of cryoprotectants (CPAs) as supplements prior to cooling and the rates of cooling/ warming of the temperature of the sample. Vitrification can eliminate intracellular ice crystals, but high concentrations of CPAs are required to achieve the vitreous state (Vajta \& Nagy, 2006; Zhou et al., 2010). CPAs function in a dual capacity by decreasing freezing temperature and increasing viscosity so that instead of crystallizing the syrupy solution becomes an amorphous ice; it 'vitrifes'. Intracellular ice crystals which organize among cooling and warming duration are the main reason for cell damage (K.-H. Lee et al., 2013). During cooling time, microscopic ice crystals appeared that may cause recrystallization in the warming process by transfer of water molecules from smaller ice crystals to larger ones, which can reach sizes that seem to be lethal for the surrounding cells. According to Hopkins et al. differences in warming rates are correlated directly with prior cooling rate because of the accumulation of tiny ice fragments in vitrified cells (Hopkins et al., 2012). A faster cooling rate is essential for a corresponding warming rate to prevent recrystallization. Coupled with moderate cooling rates, high warming rates of $117,500 \mathrm{C} / \mathrm{min}$ can protect cells from damage due to recrystallization (Peter Mazur \& Seki, 2011).

It is evident that vitrification has more benefits to offer as a cryopreservation procedure than do slow cooling methods. First, vitrification or ultra-rapid techniques prevent cryoinjuries to viable cells from ice crystal formation during the cooling process. This is due to the use of highly concentrated CPAs and very high cooling and warming rates. Second, the exposure time of the vitrification process to room temperature is shorter than for traditional freezing methods. Living cells and tissues should not remain long in such nonphysiological conditions before their storage at low temperatures. Third, vitrification is cheaper than stepwise methods. Fourth, vitrification is not requiring sophisticated machinery, and it is easy for technicians to learn vitrification protocols through a short practical training course. More over the survival rates of vitrified embryos are often reported higher than those of frozen embryos (Campos-Chillon et al., 2006; Do et al., 2014).

Although vitrification has several advantages over other cryopreservation procedures it nevertheless indicates drawbacks and disadvantages. While vitrification minimizes the time exposure of ova and embryos at room temperature (Campos-Chillon et al., 2006), this technique may lead to the contamination of embryos during the vitrifying process as a result of direct exposure to liquid nitrogen (Vajta et al., 1998). Environmental and water-borne bacteria and fungi have been identified in liquid nitrogen. It is clear that vitrification is often an open system; therefore, there is a high level of microbial contagion when vitrified samples are banked in liquid nitrogen (Morris, 2005). Hence, it is necessary to measure the contamination risks in cell and tissue banking. However, it seems that the contamination risks for cryopreserved samples are forsaken (Do et al., 2014). Vitrification methods of cryopreservation may bring new chances in research protocols. It is yet an experimental procedure. There are two major concerns about vitrification toxicity of high concentration of cryoprotectants used and microbial contamination of liquid nitrogen. The problem is to discover a protocol to successfully vitrify cells (Barun, 2015). Modifications to the vitrification process have focused on the type and concentrations of CPAs and on cooling and freezing rates which improves the vitrification process. Table 2 summarizes the advantages and disadvantages of slow freezing and vitrification techniques (Sharma, 2005). 


\section{Limitations of cryopreservation}

The metabolism of cells almost is lessen at low temperatures such as -196 @C which has inescapable side effects, such as genetic drift due to biological variations in lipids and proteins that could lead to impairmentation in cellular function and structure. If there were no restriction to the amount of utilized CPA, cells would be preserved excellently (Jang et al., 2017). In regular and standard settings, however, CPAs themselves can be deleterious for cells, particularly when used in high concentrations. For example, DMSO may alter chromosome stability which can led to a risk of tumor formation. Apart from endogenous changes in cells, the possible infection or contamination with cells should be prohibited (Jenkins et al., 2012; Yong et al., 2017). Although cryo-preservation is still undoubtedly the best present method for long-term preservation of stem cells, its disadvantages have driven research into alternatives. Cryo-preservation has long been associated with cell membrane damage and apoptosis (Trusal et al., 1984; Yong et al., 2017). Furthermore, cryo-preservation is labor intensive and operator dependent and typically uses equipment that is not suitable for cell therapy such as liquid nitrogen, which is not GMP compliant (B. Chen et al., 2013).

\section{Cell death induced by cryopreservation}

Accumulating evidence indicate that apoptotic cell death and necrosis are initiated by a variety of microenvironmental perturbations including metabolic uncoupling/coupling, ionic imbalances, energy deprivation, caspase activation, membrane phase transitions and alterations, free radical production, cellular acidosis, cytoskeleton disassembly, protein denaturation, water solidification, cell volume excursions, hyperosmolarity, and protein denaturation (Galluzzi et al., 2018; Messmer et al., 2019; Nazio et al., 2019). It has been reported fallowing cryopreservation, post-thaw apoptosis in a wide variety of cell types is a significant drawback faced by cryobiologists. Cryopreservation-Induced, Delayed-Onset Cell Death (CIDOCD) is the timing of cell death following cryopreservation which has been targeted with scientists to improve cryopreservation. It is the prime goal of researchers to generate cryopreservation solutions inhibiting signaling molecules associated with apoptosis and necrosis down the cell death cascades (Bissoyi et al., 2014). Cryoinjury-induced cell death is initiated by the intrinsic or extrinsic apoptotic pathways. According to recent studies activation of caspases 3, 8, and 9 are happened after thawing process. Various caspase inhibitors provide protection against apoptosis (Chatterjee \& Gagnon, 2001; Fowke et al., 2000). Although further study is required to provide a detailed role of caspases in the cryopreservation-induced apoptosis pathways because some studies reported caspase- 8 and caspase- 9 activity inhibition without enhancing post-thaw cell viability (Heng et al., 2007; Xu et al., 2010).

Under stressful conditions like cryopreservation, cellular oxidative stress is occurred. various reactive oxygen species (ROS) activates the multiprotein complex, including Apaf-1, cytochrome c, and caspase 9, and thus eventually leads to apoptosis.50 activation and accumulation of p53 in the cytosol through the intrinsic pathway can induces the expression of prooxidant genes at high concentrations of ROS caused by the procedure of freezing in cryopreservation (Barbaric et al., 2011). Therefore by suppressing ROS and using strong antioxidants can achieve cell recovery after cryopreservation. Also it has been reported that in the presence of the p53 inhibitor, there is a reduction in caspase 9, but not in caspase 8 activity (Melino, 2011; Sablina et al., 2005). The p38 MAPK is activated during freezing-thawing and cryopreservation processes which can induces apoptosis. P38 MAPK inhibitors can reduce apoptosis and thus led to improvement in viability of cryopreserved cells (Omori et al., 2007). During cryopreservation, alteration of actin cytoskeleton may occur and induce cell apoptosis (Bishop \& Hall, 2000). Rho-associated kinase I (ROCK-I) protein which is implicated in the regulation of apoptosis can triggers a signaling pathway that leads to actin-myosin coupling to the plasma membrane. A potent ROCK inhibitor may blocks apoptosis and improves survival of many cryopreserved cells. Figure 1. shows the mediators of cell death during cryopreservation and the effective apoptosis inhibitors (Bissoyi et al., 2014; Heng et al., 2006).

\section{Alternatives to Dimethylsulfoxide}

The standard and commonly used cryoprotective agent is dimethyl sulfoxide (DMSO), which is the cause of cell toxicity and represents some side effects. DMSO has also been recognized to show toxic effects, reduce 
the expression of key factors related to stemness, and induce epigenetic changes. Hence, there is an elevated request from health care authorities to discover non-toxic cryopreservation alternatives of DMSO (Svalgaard et al., 2018). The functions of dimethyl sulfoxide $\left(\mathrm{Me}_{2} \mathrm{SO}\right)$ as a supplement in the cryopreservation medium are to protect the cells from excessive dehydration during the freezing process and to inhibit intracellular ice formation. Due to the fact that $\mathrm{Me}_{2} \mathrm{SO}$ is toxic and associated with clinically significant side-effects as well as the fact that serum could possibly transfers pathogens, both are unsuitable for the storage of hMSC for clinical applications. Moreover, $\mathrm{Me}_{2} \mathrm{SO}$ can lead to uncontrolled differentiation of stem cells. Thus $\mathrm{Me}_{2} \mathrm{SO}$ must either be replaced for cryopreservation or it has to be removed prior to transplantation, for instance by diafiltration. Despite the possibility of removal, it is more beneficial to replace $\mathrm{Me}_{2} \mathrm{SO}$ by non-toxic agents. Non-toxic alternatives to $\mathrm{Me}_{2} \mathrm{SO}$ include, for example, glycerol or compatible solutes such as Proline and ectoin (Hegner et al., 2005; Ji et al., 2004; Woods et al., 2007).

The glycerol protection of cells from osmotic damage is represented by reducing the intracellular water loss and indeed reducing intra and extracellular ice formation (Morris et al., 2006). For cryopreservation of blood cells and adult stem cells, glycerol has already been used successfully in concentrations between 5\% and $20 \%$ (v/v) (De Vries et al., 2004; Scott et al., 2005). Ectoin was detected first in extreme halophilic bacteria (Ectothiorhodospira halocholris ) to protect these bacteria against high salt concentrations and high temperatures in their environment. Today, ectoin is also used for the stabilization of proteins and cells during the freezing process in concentrations between $0.1 \%$ and $1 \%(\mathrm{w} / \mathrm{v})$. This effect is based on the stabilization of the hydrate envelope of enzymes and the regulation of the osmotic balance in cells. Proline is a nonessential amino acid and a special cryoprotectant, because it has low- and macromolecular behavior. Proline can diffuse into the cells and forms hydrogen bonds typical for low-molecular cryoprotection. Moreover, depending on the concentration, proline forms aggregates which act like macromolecular cryoprotectants (Grein et al., 2010; Troitzsch et al., 2008). Proline can preserve enzyme activity and membrane stability after freeze-thaw cycles (Rudolph \& Crowe, 1985) and increases the post-thaw vitality of spermatozoa in concentrations between $5 \mathrm{mM}$ and $20 \mathrm{mM}$ (Y. Li et al., 2003). In a study which ectoin was the cryoprotectant, the results indicated high post-thaw cell survival of up to $72 \%$ whereas after cryopreservation with glycerol and proline, the hMSC cells were completely dead or had only poor cell survival. Indeed, following the cryopreservation with proline, the altered morphology of cells was seen. These results show that glycerol and proline are not appropriate for cryopreservation of hMSC. In contrast, ectoin has the qualification for being the alternative of Dimethylsulfoxide in a serum-free cryomedium (Grein et al., 2010). Pentaisomaltose as an extracellular cryoprotectant is agreed for clinical utilization. It is less likely than DMSO to interact with the intracellular molecules. Cryopreservation in DMSO or pentaisomaltose have been compared in cell survival in vitro, also in long-term cryopreservation in the in vivo models (Svalgaard et al., 2018).

\section{Alternatives to Cryopreservation:}

\section{Dry preservation}

One of the alternatives for cryopreservation is elusion of low temperature storage and transport by desiccating cells which could be kept at room temperature. If possible this would be economically and practically good alternatives for long-term storage of cells (Coopman, 2013). Freeze drying (lyophilisation) is useful for preservation of bacterial cells and fungi. Briefly, the process comprises three stages: freezing in order to partly crystallise the solvent so that it can be separated from the solutes, sublimation of the resultant ice (primary drying phase) and finally, a secondary drying phase where the majority of the remaining moisture is removed by desorption (Sundaramurthi \& Suryanarayanan, 2012; Wolkers et al., 2002). Just as cryoprotectants are used in cryopreservation, cells can be pre-treated and indeed lyophilised in the presence of lyoprotectants, to minimise cell damage during the process. One compound that has been particularly researched is trehalose, a hydrophilic disaccharide which is known to accumulate in desiccation tolerant organisms (Crowe et al., 2005).

According to Watanabe K. et al. studies; Pv11 was the first dry-preservable animal cell line which showed improved survival and achieved proliferation after dry storage. The method is mentioned in figure.2 including preincubation, desiccation and rehydration processes. As a result, they managed to obtain successful 
drying and storage of Pv11 cells at room temperature by preincubation in trehalose solution for $48 \mathrm{~h}$ before dehydration to achieve successful dry preservation of Pv11 cells. They extended this dry storage up to 251 days and the cells still kept nearly $7 \%$ viability (Watanabe et al., 2016).

\section{Hypothermic Preservation}

Hypothermic preservation is pausing of cells at hypothermic temperatures $\left(1^{\circ} \mathrm{C}-35^{\circ} \mathrm{C}\right)$ (J. Yang et al., 2017). It is another potential alternative to cryopreservation for mammalian cells where cells are stored at low but not freezing temperatures, effectively pausing the cells by slowing down metabolism, but without the dangers of ice formation. At mild hypothermia $\left(25-35^{\circ} \mathrm{C}\right)$ cells proliferation is still seen and recombinant protein expression may be improved by culturing cells at reduced temperatures (Miyamoto et al., 2009). More severe hypothermia and refrigerated storage $\left(0-10^{\circ} \mathrm{C}\right)$ is needed to arrest growth and pause the cells but this would nonetheless be preferable to storing cells in liquid nitrogen and the use of dry shippers. This would be a short term preservation method and indeed, it is already used in the pharmaceutical industry as it can uncouple cell culture from cell-based assays. It allows researchers to store cells for several days if a piece of equipment is double-booked or breaks down, rather than having to dispose of plates of cells, saving time and money (Coopman, 2013; Wise et al., 2009).

\section{Agarose-Gel Based Method}

In the state of cell transportation, agarose was extensively utilized in viral plaque assays at concentration of $0.8 \%$. Rigidity might be a main factor for supporting adherent cells and prevention of cells. For this reason the agarose-medium gel should have enough rigidity. One of the important conditions for the utilization of agarose-medium gel method is the temperature adjustment. It should be between 1 to $37^{\circ} \mathrm{C}$ since greater than $37^{\circ} \mathrm{C}$ or lower than $0{ }^{\circ} \mathrm{C}$ can be damageable for cells. On the other hand agarose concentration should be adjusted between 0.8 to $1.2 \%$. Because the agarose layer at concentration less than $0.8 \%$ is not hard enough and at the concentration above $1.2 \%$, it becomes dry and easy to crack. The higher concentrations of agarose (higher than 1.2\%) might be the reason for low cell viability. Thus, agarose concentration at $1 \%$ is an optimal condition for this cell transportation method because it has the highest cell recovery viability and is firm enough to form an appropriate layer of gel to support the cell during the transportation.

According to Yang L. and et al study on the agarose gel based method, the numbers of cell colonies were detected for testing the viability and function of cells after transportation. Cell morphology which were being kept in medium containing agarose gel at room temperature were similar to those in regular cell culture condition. The results of colony formation detection indicated that the morphology and size of cell colonies for three cell lines were not changed after recovering from the transportation by this method (L. Yang et al., 2009).

Wheatley S. P. et al. represented a gel based method which provided transit of up to 2 weeks without compromising cell recovery. Its application will help scientists and permit them to send cultured cells across the world without requiring dry-ice and at ambient temperatures. In this research privileged recovery is observed when cells are transported at $5 \times 10^{6}$ cells $/ \mathrm{ml}$ at the temperature of $4-27^{\circ} \mathrm{C}$ for 1 week. They counseled to ship cells in small aliquots of Transporter $(100 \mu \mathrm{l})$ in microfuge tubes and diffuse the gel pellet prior seeding (Wheatley \& Wheatley, 2019).

\subsubsection{HemSol gel}

From a development viewpoint, a truly "ready to go" cell system, shipping of both suspension cell and adherent cell cultures at ambient temperature is now possible. Cells can be prepared in a multi-well plated, packaged with HemSol gel and sent to end users. According to Stefansson S. and et al studies, HemSol gel can protect platelet and red blood cells function in cold storage up to 6 days. They also analyzed live cells' function during transportation with HemSol gel method. End users will receive functional cells and already plate to perform assays. Thus, HemSol gel is a noteworthy alternative to standard dry ice shipment and could develop the utilization of cells in pharmaceutical and biotechnology studies. The cells which shipped in HemSol gel represented more than $95 \%$ viability and restored biological functions in 2 hours, whereas, 
cells that shipped in dry ice needed more than 24 hours to recover and indeed they required media change for removal of the DMSO (Stefansson et al., 2017).

\section{Polymer Based Cryogel Matrix}

Recently, matrices synthesized at subzero temperature using cryogelation technology known as cryogel have been reported for cryopreservation (Katsen-Globa et al., 2014; Vrana et al., 2012). These polymeric cryogels possess three-dimensional (3D) structure and have already been used as a scaffold for tissue engineering applications (Hanna \& Hubel, 2009). Cell-scaffold construct for regenerative medicine was culturedin vitro which was then transplanted in in vivo (Sarkar \& Kumar, 2016; Umemura et al., 2011). Pausing or storing of the developed tissue engineered construct is prohibited during this entire process. Therefore, cryopreservation of cell-scaffold construct having viable and functional cells is a preferable approach to meet the demand. Due to their ready-to-use nature, they could be immediately utilized. These cryogel-based methods for cryopreservation have advantages over the gel-based method because in the former cell encapsulation and gel formation do not occur simultaneously (Vrana et al., 2012).

Cryogels were preferable over traditional gel carriers in the field of biochromatography and related biomedical studies. These matrices approximately simulate the three-dimensional structure of native tissue extracellular matrix. Indeed, mechanical, osmotic and chemical stability of cryogels make them wonderful polymeric materials for the construction of scaffolds in tissue engineering investigations and in vitro cell culture, immobilization of biomolecules, capturing of target molecules, and controlled drug delivery. Cryogels are known as gel matrices which are produced by polymerization at low temperatures (below zero). While the polymerization process happens in the unfrozen part, ice crystals perform as pore forming agents. Figure 3 represents the Schematic view of cryogel formation and Figure 4 shows the physical characterization, digital image and SEM image of HEMA agarose (HA) and gelatin cryogels (Bakhshpour et al., 2019).

Bioreactors are specified as devices which protect biological reactions. They have a significant role in the cell cultivation providing control and monitoring of $\mathrm{pH}$, temperature, oxygen and nutrient decomposition in microbiology. When tissue engineering is taken into consideration, bioreactors can be applied to develop new functional tissue in vitro with the production of regulatory signals in an appropriate physical environment. In several research publications, it has been mentioned that the cryogels have vital function in bioreactor platforms. A research about the storage and transportation ability of the cells have reported the utilization of both HEMA-agarose (HA) and gelatin cryogels for this aim. These materials also can be applied in cell cultures for both in vitro and in vivo studies. In these conditions, they influence the improvement of cell migration, proliferation and differentiation as well as enhanced mechanical stability (Bakhshpour et al., 2019). Scanning electron microscope (SEM) analysis of both the cryogels were performed for surface morphological analysis. The homogenous pore distribution was observed under SEM. Presence of pores associated in easy recovery of cells. Hence, pore walls provide adherent surface to the cells and help for cell proliferation (Kumari \& Kumar, 2017).

Kumari J. and et al have tested the possibility of using cryogels for cell transportation. According to this study cryogels could be integrated with the methodology of using cryogel for cryopreservation. Cells received from the cryogel were functional and viable following the simulated transportation using 2-hydroxyethyl methacrylate (HEMA)-agarose (HA) cryogel. Further, we have also shown that the cells stored in the gelatin cryogel remain fit for cryopreservation even after the simulated transportation for up to five days. The results show that cryogels not only overcome the above-mentioned problems of transportation of cells but also provide a ready-to-use scaffold for further engineering of adherent cells, including cryopreservation. Application of cryogels for transport and storage also shows another advantage such as well cell proliferation during transportation. Thus cryogels could be a preferable solution for tissue engineering including celltransportation and cell-cryopreservation (Kumari \& Kumar, 2017).

In summary, HA and gelatin cryogels have the potential of transporting and storing adherent-cells. As both the cryogel have different surface properties, they can be used for various applications. Kumari J. and et al had produced HA cryogel for the purpose of cell transportation with the chance of cells that can be eluted 
following the transportation. On the other hand, they suggested gelatin cryogel that can be utilized for both cell transportation and cryopreservation. In both, cells were alive and had the capability of proliferation after simulated transportation. The adherent-cells kept in HA cryogels and C2C12 cells remain up to $25 \%$ more viable in comparison with the adherent-cells kept in cryovial as a suspension culture under the same simulation conditions for transportation. It is demonstrated that the adherent-cells can be stored in gelatin cryogels and $\mathrm{C} 2 \mathrm{C} 12$ cells, up to five days at room temperature and representation viability up to $80 \%$. It has further shown that these adherent-cells indeed remain fit for cryopreservation even after the simulated transportation in cryogels for up to five days. This suggests that cryogels can minimize the effect of shear force, which is the main cause for the reduction of viability in transporting cells in suspension. Cryogels act as an efficient, low-cost storing and transporting matrix at room temperature and at cryo-conditions, and hence it may show serious outcome for ready-to-use transplantation, in vitro drug testing, and regenerative medicines. These pave the way for establishing cryogels as a superlative solution for tissue engineering, including cell-storage, cell-transportation, and cell cryopreservation (Kumari \& Kumar, 2017).

According to some investigations, transportation of mammalian cells for long distance at ambient temperature can be performed through agarose gel-or matrigel-based methods. Also it is shown that these procedures retain a high rate of cell recovery after transportation for a few days. These procedures are complex and labor-consuming. Whether mammalian cells to be shipped in a simple mode at ambient temperature remains obscure (Junjian Wang et al., 2015; L. Yang et al., 2009). Novel polymer based cryogel matrix method is simple, practical and affordable because specific equipment and commercial medium aren't needed. In this method cells were directly suspended in the culture medium. It can be used for cell shipping at short and long distances. However, it should be performed carefully and cautiously for the shipment on clinical purpose where specific equipment and techniques are highly required (Wille et al., 2014). It not only expands the comprehension of the effect of hypothermia on cell survival, but also provides an alternative approach for cell shipment. According to this method, the approach for cell shipment would be useful to biological and biochemical laboratories (Juan Wang et al., 2017).

\section{Encapsulation Method}

Cell microencapsulation technology is an important technique for prevailing over the bioprocess challenges. In this process cells are microencapsulated in alginate to progress a unified bioprocess for cryopreservation of pluripotent hESCs. The existence of components of the extracellular matrix on microcarrier cultures (e.g. collagen, laminin) may have participation with cell survival enhancement during freezing and thawing (Ji et al., 2004; Kim et al., 2004), by reducing post-thaw apoptosis. The alginate microcapsule permits extra increase of post-thaw cell viability up to 3 times more compared to non-encapsulated cultures. Although the underlying mechanisms are still obscure, several experiments demonstrate that maintaining cell-cell/matrix contacts improves hESC recovery following cryopreservation (Hunt, 2007; Karlsson \& Toner, 1996). Cell entrapment within alginate microcapsules may help assist hESCs in the presence of harmful effects of cryopreservation, by preventing the disruption of cell-cell and cell-matrix contacts, decreasing exposure to cryoprotectants and preventing the damage caused by intracellular ice formation (Malpique et al., 2010; Zimmermann et al., 2005).

Hydrogels such as alginate, poly (lactic-co-glycolic acid)/ poly (l-lactic acid) scaffolds, agarose, chitosan and hyaluronic acid have been used in hESC culture. Alginate is the most commonly used encapsulation material owing to its biocompatibility, biosafety and permeability. Although Nie et al reported a novel technique for the cryopreservation of hESCs adherent on microcarriers, this protocol required more optimization in order to omit animal feeder cells and enhance cell attachment/survival after thawing (Serra et al., 2011).

Recently the frequent application of hydrogels as cell carriers for delivery of stem cells are now known to be challenging conventional cryo-preservation methods (Gorodetsky et al., 2011). The cell samples used for study of alginate hydrogels as cell carriers were human mesenchymal stem cells (hMSC) and mouse embryonic stem cells (mESC). These cells were successfully stored inside alginate hydrogels for 5 days under ambient conditions in sealed cryo-vials. After extraction of cells from alginate gel, cell viability results show $74 \%$ survival rates for $\mathrm{mESC}$ and $80 \%$ for hMSC, which were compared with survival rate results gained following 
cryo-preservation. Indeed, the proliferation rate and detection of mRNA and protein levels in hMSC and mESC extracted from alginate hydrogels were also comparable to cryo-preservation (B. Chen et al., 2013).

Stem cells in suspension do not survive for extended time intervals during transportation under ambient conditions (Reddig \& Juliano, 2005) within appropriate delivery times and at reasonable financial cost. Hence, cryo-preservation of stem cells was previously known invalid owing to the adverse effects of DMSO (Chakrabarti \& Schutt, 2001). Thus, appropriate conditions for the preservation of transported stem cells are demanded. A hydrogel that is chemically inert, structurally uniform and biocompatible, may be useful for a simple and economical storage method which subjects stem cells to minimal manipulation and preserves their viability and phenotype (B. Chen et al., 2013).

The major properties of alginate based cell encapsulation such as biocompatibility and rapid gelation provide the success of cell encapsulation. The mechanical properties of alginate hydrogels are mainly related to the ratio of components used for gelation including polysaccharides and the cationic cross-linker (Martinsen et al., 1989). Although calcium is the conventional crosslinking ion for alginate gels, other cations such as strontium and barium are appropriate (Mørch et al., 2006; Place et al., 2011). Strontium-alginate microbeads were known to be more stable than calcium alginate beads (K. L. Chen et al., 2007). The mechanical properties of alginate gels in the presence of live cells also can be controlled by modification of the internal porosity of these gels. The addition of hydroxyethyl cellulose to alginate, led to gels with controllable pore size, which relate to the improved viability of cells immobilized within this scaffold (Wright et al., 2012).

Having found that calcium alginate gels were too fragile, and agitation would release the encapsulated cells resulting in their death. We surmised that the transport of cells would cause significant agitation to the gels resulting in their disintegration. Therefore to avoid possible alginate hydrogel fragmentation during long distance transportation, strontium was chosen to cross-link with alginate gels in this study. Previously strontium was found to have a greater affinity to alginate than calcium, resulting in alginate hydrogels with improved stability. Strontium alginate hydrogels have successfully been used in bone tissue engineering. We further improved the gels overall mechanical strength by encapsulating within it an inert nylon mesh. Together the composite material, comprised of strontium alginate gel and nylon mesh, produced an extremely robust and effective carrier for hMSC and mESC. Chen B. et al. results clearly show that both mESC and hMSC stem cell markers were positive following extraction from the alginate gels remarkably even after 5 days at ambient (room temperature) in a sealed cryo-vial (B. Chen et al., 2013).

According to Swioklo S. et al. investigation alginate-encapsulation method was applied to improve hypothermic preservation of human adipose-derived stem cells. They evaluated the essential conditions required for hASCs cell survival and function over short-term periods in hypothermic storage. Due to inert hydrogel characterization of alginate, cells are separated from each other resulting in little or no physical cell-cell interaction that may offer protection at higher temperatures. After 24 hours encapsulation in culture, it was not detected any change in metabolic activity per cell. The cytoprotection was maintained in cell densities of up to $2 \times 10^{6}$ cells $/ \mathrm{mL}$, resulting in $70 \%$ viability. Assessment of Morphology and proliferative potential of hASCs following storage showed that Growth kinetics had not changed in all samples up to day 6 . In this research it was demonstrated that cell density could have an effect on viable cell recovery (Stacey et al., 2017; Swioklo et al., 2016).

\section{Fibrin Microbeads}

Fibrin microbeads (FMB) are human fibrin-based, dense, nonimmunogenic, and slowly biodegradable particles with prolonged shelf life that were developed as cell carriers. FMB is a simple and highly qualified tool to isolate mesenchymal stem cells (MSCs) from different mixed sources and to support the expansion of many matrix-dependent cells in a three-dimensional (3D) culture with potential application in cell-based regenerative medicine. Cells which were loaded on FMB and cultured in slowly rotating 3D conditions in suspension can attain a high density (Ben-Ari et al., 2009; Gorodetsky et al., 1999; Shimony et al., 2006).

A number of other biodegradable microspheres and microcarriers were suggested for cell culturing in 3D (Chung \& Park, 2009; Kang et al., 2008; Park et al., 2011), but they do not represent multifunctional features 
and do not provide stem cell separation, expansion, and support them while differentiating at the same time. They are acting as the carrier for cell implantation. According to one study, FMB-based technique was used for delivery of mesenchymal cells and other matrix-dependent cells in sealed vials at room temperature for long time intervals of $>10$ days. They reported high survival rate, without any need for further supportive infrastructure. The role of a possible protective mechanism of MSC attached to FMB by hypoxia induced factor (HIF)-1 is discussed (Gorodetsky et al., 2011). They tested the rate of proliferation for the cells after room temperature storage. They reported that after recovery time, cells keep their properties which was performed before and after 6 days in room temperature storage. The results displayed that the cells not only survived well but also have the same proliferation rate. Figure 5 represents the setup of cells cultured on fibrin microbeads (Gorodetsky et al., 2011).

\section{Osmolyte Solution Composition}

Natural osmolytes like sugars, sugar alcohols and amino acids work by stabilization of biological systems exposed to environmental extremes. Raman images shows the influence of osmolytes on ice crystal shape, which reflects the interactions between osmolytes and water. C.-H. Pi et al. optimized DMSO-free cryoprotectants for Jurkat cells as a model CD3+ T cell. They applied mixtures of osmolytes formulations of SGI (sucrose-glycerol-isoleucine), TGI (trehalose-glycerol-isoleucine) and MGI (maltose-glycerol-isoleucine). They designed a comparative study to evaluate the effects of three DMSO-free osmolyte-based formulations to provide better awareness to the potential of these cryoprotectants in terms of preserving a heterogeneous population. DMSO-free cryoprotectants indicated competitive post-thaw recovery of PBMCs (CD45+) and higher preservation capability for primary $\mathrm{T}$ cells and helper $\mathrm{T}$ cells. In their previous work they represented the effect of combining osmolytes on preservation quality and characterized the cell and osmolytes interactions on Jurkat cells. They detected that multicomponent osmolyte solutions including sugars, sugar alcohols and amino acids were effective in cryopreserving cells (C.-H. Pi et al., 2020; C. Pi et al., 2019). In the other study they characterized the "sweet spot" for the preservation of a T-cell line using osmolytes (C.-H. Pi et al., 2018).

Traditional knowledge designates that appropriate cryopreservation includes the control of ice formation in the freezing process. The thermophysical properties of each cryoprotectant solution were evaluated and discovered that the formulation of trehalose-glycerol-isoleucine had the lowest enthalpy of melting, indicating less ice formation. Although this formulation was not the optimal cryoprotectant for every cell type, it proved that the mass of ice formation in the freezing process is not the unique factor for cell recovery. Biology is a contributing factor of the cryopreservation process. The results indicate that the biological interactions between cryoprotectants and water and also between cryoprotectants and cells can associate with improvement of the recovery of cell types which are mainly sensitive to cryopreservation. These cell types have various responses to cryoprotectants obviously, and there may not be a global cryoprotectant that can optimize all branches at the same time. Finally, understanding the knowledge of freezing responses of different subsets of PBMCs will permit for more potent and successful cryopreservation of PBMCs (C.-H. Pi et al., 2020).

\section{Trehalose-Contained Solution Formula}

Trehalose is disaccharide of glucose which has the ability of stabilizing and preserving cells or tissues through the freeze and thaw procedures (Erdag et al., 2002). Albeit the mechanisms were unknown, it could be used alone as an excellent alternative CPA in the cryopreservation of red blood cells and germ cells (Eroglu et al., 2000; Satpathy et al., 2004). A few studies have investigated trehalose ability to keep the cells safe from apoptosis in relatively high temperature (i.e., 48C). In a study, the effect of trehalose was evaluated on improvement of the survival of MSCs at 48C. Di G. et al developed a solution formula that could effectively preserve MSCs at 48C for up to 2-3 weeks (Di et al., 2012).

Trehalose is known as a nontoxic compound which has been commonly used as natural CPA for cryopreservation of cells and hypothermic storage of human tissues (Erdag et al., 2002; Pu et al., 2005). It was demonstrated that the addition of trehalose to the cryopreservation medium remarkably increased the sur- 
vival of human hepatocytes and amniotic fluid-derived stem cells (Katenz et al., 2007; Seo et al., 2011). Also, the low concentrations of intracellular trehalose could increase the survival rate of human cells during cryopreservation (Eroglu et al., 2000; Satpathy et al., 2004). However, the exact mechanism of efficient preservation of biological systems by trehalose during freezing and drying is obscure. It is believed that trehalose has the ability to stabilize phospholipids and proteins through its direct interaction with polar groups of these biomacromolecules during freeze-thawing process (T. Chen et al., 2001; Crowe et al., 2003).

Cryoprotectants are classified into two types of Penetrating and nonpenetrating (McGann, 1978). The penetrating types comprise glycerol, 1,2-propanediol and dimethyl sulfoxide. The nonpenetrating cryoprotectants involves polyvinylpyrrolidone, trehalose, fructose, sucrose and glucose. Trehalose is found in numerous organisms, such as nematodes and yeasts, which are capable of surviving during freezing and drying. Previous studies have used trehalose for the purpose of human cell cryopreservation such as platelets, red blood cells, sperm, oocytes, pancreatic islets and fetal skin. Hence, the administration of an alternative protocol with trehalose for cryopreservation of stem cells obtained from umbilical cord blood and bone marrow has previously been reported. Also in a study by Scheinkonig et al. cryopreservation of stem cells obtained from mobilized peripheral blood stem cells has been reported. Conversely, in another study, trehalose was used to cryopreserve pure Hematopoietic stem cells and progenitor cell (Beattie et al., 1997; Erdag et al., 2002; Eroglu et al., 2002; Martinetti et al., 2017; Motta et al., 2014; Scheinkönig et al., 2004; Wolkers et al., 2001; Zhang et al., 2003).

The application of the anhydrobiotic compound trehalose (a,a-trehalose, a-D-glucopyranosyl-a-Dglucopyranoside) has been known as a cryo/lyoprotective agent with or without other CPAs for a wide variety of therapeutic applications ranging from blood banks to reproductive technology (Eroglu et al., 2000; Y.-A. Lee et al., 2013) Trehalose forms hydrogen bonds with biomolecules and preserves their functional conformations during water deficiency (Lawson et al., 2011). Indeed trehalose acts as a nonpermeating molecule to form a stable glassy matrix with low molecular mobility that suspends any intracellular degradative biochemical processes in response to water loss by prohibiting the resultant lipid phase transition (Hara et al., 2017).

Adding relatively low concentrations of dimethyl sulfoxide (DMSO) and trehalose as cryoprotective agents (CPAs) for the evaluation of their synergistic effect on long term cryopreservation process was subjected by some studies (Beattie et al., 1997; Choi \& Chang, 2003; Yokomise et al., 1995). Lee L. Q. Pu et al. suggested a cryopreservation method containing trehalose as a cryoprotective agents. They developed a practical technique by adding trehalose alone as CPAs. It considered to represent better long-term preservation method for adipose tissues because the viability rate of cells was significantly increased. Further studies are needed to refine their method for cryopreservation with trehalose as a CPA. Since non-toxic characteristics of trehalose, it can be used alone, as a single CPA, in long-term preservation of adipose tissues and the removing stage does not important (Pu et al., 2005).

\section{Sucrose-Based Media}

Non-DMSO cryoprotectants such as sugars have been commonly utilized to be an alternative of DMSO in the cryopreservation of various mammalian cell types. According to research results sugars can be effective in combination with sugar alcohols and amino acids in the cell preservation process. Numerous studies have been performed to figure out the mechanisms of protective effects of sucrose, most of which aimed at the interactions between sucrose and water, protein and membranes. Yu G. et al. explored the interactions between sucrose and water in a frozen sucrose solution. They visualized the interactions between sucrose and cell membrane at a low temperature directly and the mechanisms of its protective effects on cells. They detected the strengthened hydrogen bond network in concentrated sucrose solution. They suggested that protective properties of sucrose might originate from its direct interaction with cells. Recently, the high spatial resolution of Raman spectroscopy allowed us to visualize the distribution of extracellular and intracellular ice, cryoprotectants and the size of ice crystals formed in different combinations of cryoprotectants during freezing time. They quantified the variation in the sucrose concentration in a gap between a cell and the ice phase by using Raman spectroscopy images. The sucrose concentration at the interface between cell and 
extracellular ice was lower than that in bulk nonfrozen solution (Yu et al., 2018). Shanina I. V. et al. had a study on comparison of a sucrose-based solution with other preservation media in hepatocyte cells. According to their results, sucrose-based solution could be a less complex alternative solution for cold storage of isolated hepatocytes. The stability of liver cells at low temperature were conducted by exclusion of trypan blue dye and morphological appearance. Dye exclusion in sucrose-based solution was higher than in other mediums (Shanina et al., 2000).

\section{Collagen-Based Scaffold}

Cell interaction with scaffolds occurs via chemical groups or ligands on the material surface. Natural extracellular materials (e.g., collagen) are the Precursor of scaffolds. The ligands of Arg-Gly-Asp have naturally found in the material surface. The pore size of a scaffold shows an essential component for cell attachment, survival, and function (Daei-farshbaf et al., 2014; Ranucci et al., 2000). Porosity and the water-uptake capacity of the scaffold are also vital parameters in cell-scaffold interaction. Custom-made scaffolds frequently lack reproducibility. Ruo M. et al after characterization of the scaffold, shipped cells on scaffold over 10 days. Optimaix-3D was the name of a scaffold which was reproducibly manufactured in a controlled freezing process from a defined collagen dispersion (Schoof et al., 2001). Primary human hepatocytes need the interaction with Extracellular matrix (ECM) components, such as collagen, to maintain their specific functions. The ECM protein collagen presents a significant function in the maintenance of organs and tissue (Baiocchini et al., 2016). So it is known as a biomaterial utilized in medical and bioengineering sciences. It is biodegradable and in contrast to albumin or gelatin only weakly antigenic (C. H. Lee et al., 2001). Collagen is also one of the vital ECM proteins of the healthy liver. Therefore, the Optimaix-3D scaffold could be an appropriate carrier for primary human hepatocytes for transportation and/or metabolic studies (Khan \& Khan, 2013).

According to Ruo M. et al studies, the viability of the cells can be maintained significantly better by transport on the Optimaix-3D Scaffold from Matricel compared to the conventional shipment as a cooled suspension. Additionally, the utilization of Optimaix-3D Scaffold led to maintenance of the important hepatic functions including drug metabolism, urea production, and albumin synthesis over the period of 10 days. Hence, the scaffold has nearly the same stiffness as a healthy liver. With its high porosity and permeability, it is not only ideal for supplying the cells with nutrients, but also for use within a bioreactor. Due to the good biocompatibility of the collagen used for the scaffold, it might be also possible to use a scaffold seeded with primary human hepatocytes in regenerative medicine (Ruoß et al., 2018).

\section{Natural Zwitterionic Betaine}

Betaine can perform as a highly promising CPA. Betaine is a zwitterionic and hydrophilic molecule rich in abundant microorganisms, plants, and animals. Betaine has efficient effects on ice formation and can regulate osmotic stress. Betaine is a well-known osmoprotectant capable of protecting cells against osmotic stress (Courtenay et al., 2000). Also zwitterionic betaine could produce a monolayer of water around the proteins to maintain their stability and functions. Moreover betaine is related with the freezing tolerance of various plants. According to Kishitani et al. studies the winter-type barley by accumulation of betaine at high levels in their leaves during cold acclimation can protect them from freezing injury (Nomura et al., 1995). Yang J. et al. represented cell cryopreservation using betaine as a nontoxic alternative to DMSO. In order to their findings ultra-rapid freezing was more straightforward than the conventional controlled-rate freezing protocol due to diverse cell types cryopreservation, better post-thaw survival efficiency and low cytotoxicity (J. Yang et al., 2016).

The interaction of water molecules had shown an exact relation with ice formation. For this reason for minimizing the ice formation, CPAs are commonly hydrophilic molecules indicating strongest inhibition to water crystallization and excellent capability of decreasing the water chemical potential. Zwitterionic betaine is well known for its highly hydrophilic property; it can strongly bind water molecules via ionic solvation effects due to its charged groups (Shao \& Jiang, 2015). As well as ice injury, solute injury occurred by osmotic stress is also responsible for cell death during the freezing and thawing process. Following cells were exposed in hypertonic betaine medium, they could still assume their spindle shape was similar to the control 
cells. Interestingly, adding betaine into the hypertonic $\mathrm{NaCl}$ medium was able to significantly improve the viability of cells. These events indicated that by accumulating this natural osmoprotectant named betaine, cells would adapt to external osmotic stress. Therefore, betaine highlighted the uniqueness for protecting cells from solute injury. This natural zwitterionic molecule is a nontoxic CPA and enables cells to survive ultrarapid cryopreservation. Cellular uptake of betaine was ultra-rapid for intracellular protection during the freezing process. Due to betaine privileged cell cryopreservation ability, it has the possibility to be an alternative CPA for the conventional toxic ones (J. Yang et al., 2016). Figure 6 proposes the mechanism of cell cryopreservation using betaine with ultrarapid freezing.

\section{Bio-Inspired Cryo-Ink}

One of the important challenges in conventional vitrification is ice crystallization, which happens during rapid rewarming. It occurs as a result of the latent heat of fusion, causing hemolysis of the cells. It has reported a new technology-enabled capability of printing viable and functional cells. To avoid such crystallization, Assal R. E., et al applied a novel and innovative cryo-ink unified with a cryo-printer that can transform a bulk volume of human blood into nanoliter droplets on a cryo-paper. In this method, the cryo-ink solution includes ectoine, trehalose, and polyethylene glycol. The cryo-ink as a cryoprotective agent can assist the cells overcome the shock until the cooling and rewarming processes of cryopreservation. The cryo-ink solution used in this study posed complementary enhancing effects. Ectoine is present in the extremophilic bacteria which has the ability of adapting to extreme thermal and osmotic stress conditions. Indeed PEG suppresses the freezing point of the solution. Ultra-rapid rewarmation through immersing the printed nanoliter droplets on cryo-paper in warm media ([?]37 degC) can avoid crystallization (Figure 7). Furthermore, utilizing bio-inspired CPAs; such as ectoine and trehalose, in low concentrations eliminate extensive washing steps. Assessment of RBC morphology following ectoine-based vitrification and rewarming indicated that approximately $88 \%$ of RBCs during the ectoine-based vitrification maintained their original form with low irreversible morphological alterations compared to low-glycerol slow freezing and low-glycerol vitrification methods. Furthermore they measured band-3 phosphorylation, CD35 expression, intracellular nitric oxide (NO), and intracellular reactive oxygen species (ROS) for evaluating the functional properties of EV recovered RBCs (El Assal et al., 2014).

As a result of using DMSO, a usual toxic cryoprotectant, current cryopreservation protocols intail deterioration in NK cell viability and functionality which associated with side-effects in human. For this reason in another recent research which performed by Assal et al., the human natural killer cells were cryopreserved by slow freezing method using a cocktail of biocompatible bioinspired cryoprotectants including dextran and carboxylated $\varepsilon$-poly-L-lysine. They assessed morphology of RBC following ectoine-based vitrification and rewarming (Figure 8). It is important to notice that the anti-tumor functional activity and viability of recovered NK cells assessed and demonstrated higher cytotoxic potency against leukemia cells compared to cells cryopreserved with DMSO based solutions (El Assal et al., 2019). Dextran acts as a cryoprotective for various cell types. Carboxylated PLL similar to antifreeze proteins (AFPs) inhibits ice crystal growth and recrystallization during cryopreservation. By combining both dextran and carboxylated PLL, they showed synergic cryoprotective effect. These molecules indicate high affinity to water and thus could also they have the ability to remove intracellular water during freezing. The combination of dextran and CPLL could also interact with concentrated salts controlling the degree of dehydration to a level sufficient to avoid intracellular ice formation during freezing (Jain et al., 2014).

\section{Discussion:}

It is inevitable that for research studies, the mammalian cells have to be shipped globally from one laboratory center to another (Juan Wang et al., 2017). According to some ancient cell shipping procedures they need a special container and dry ice or need to fill flasks of live cells with medium. In comparison, a agarose-medium gel based method for transporting live cells directly in a plate (e.g. 6-well plate) has advantages of simple, low priced, no requirements for special container and dry ice, and no liquid in the cell plate. Cells shipped in live condition have a fast recovery process following the receiving time compared with the traditional methods. Therefore, agarose-medium gel based method is a helpful and convenient technique for cell transportation 
between laboratories around cities or countries. In this method the appropriate time for cells transporting should be in 3 days (no more than 5 days) following the coverage of the cells with the agarose-medium gel (L. Yang et al., 2009).

A novel and easy utilization of alginate hydrogel encapsulation, may offer an inexpensive and powerful alternative to cryo-preservation for the transport and storage of stem cells for clinical and research studies. The appropriate storage duration for stem cells encapsulated within alginate gels is about five days under ambient conditions in an air-tight environment. This method also affords to allow the easy recovery of cells, and retain their viability and phenotype. It is a practical technique for the transport of stem cells, which will be beneficial for clinical and research studies (B. Chen et al., 2013).

Cells sealed on Fibrin microbeads at room temperature were protected, but did not represent any elevation of their hypoxia-induced factor-1a expression. Although the density of cells sealed on Fibrin microbeads incubated to reach confluence, the density of cells sealed on Fibrin microbeads at room temperature did not alter even after 10 days at room temperature. After 6 day storage of cells on Fibrin microbeads at room temperature, no alteration of $\mathrm{pH}$ and gas level were detected in the medium. Fibrin microbeads are types of cell carriers which could be useful for transferring progenitor cells at room temperature for long term intervals between different research centers (Gorodetsky et al., 2011).

Development of non-toxic and animal serum-free preservation medium is an important challenge for storage and distribution of mesenchymal stem cells. Therefore, it is inevitable to develop a formula that could preserve MSCs at $48 \mathrm{C}$ for up to 3 weeks. There is a formula which says trehalose is the important ingredient for maintaining viability of MSCs. Cells preserved in the formula contain trehalose still representing $70 \%$ viability for 3 weeks and they are similar to those of freshly harvested mesenchymal stem cells in terms of growth kinetics, expression of cell surface antigens, and differentiation potential. In summary, storage of MSCs in the medium makes it far easier for transporting the cells from processing units to clinical sites (Di et al., 2012). In particular, the efficacy of trehalose has been reported for clinical purposes in blood stem cells. The aim of the current study was to establish an efficient method for biological research based on the use of trehalose, to cryopreserve pure peripheral blood stem cells. The efficacy of trehalose was assessed in vitro and the cell viability was evaluated. The results showed that trehalose could improve cell viability after thawing compared with the standard freezing procedure. These data could confirm the potential of trehalose application for cell cryopreservation studies in future (Martinetti et al., 2017). Indeed the effect of combining osmolytes on preservation quality indicated that multicomponent osmolyte solutions including sugars, sugar alcohols and amino acids were effective in cryopreserving cells (C.-H. Pi et al., 2020; C. Pi et al., 2019). Numerous studies have been performed to figure out the mechanisms of protective effects of sucrose at low temperature of preservation. Recently, the high spatial resolution of Raman spectroscopy allowed us to visualize the distribution of extracellular and intracellular ice, cryoprotectants and the size of ice crystals formed in different combinations of cryoprotectants during freezing time. They quantified the variation in the sucrose concentration in a gap between a cell and the ice phase by using Raman spectroscopy images. The sucrose concentration at the interface between cell and extracellular ice was lower than that in bulk nonfrozen solution. It is suggested that protective properties of sucrose might originate from its direct interaction with cells (Yu et al., 2018).

The Optimaix-3D Scaffold is appropriate for Primary human hepatocytes long-term culture and transport. The novel collagen-based scaffold is characterized by its pore size, porosity, water-uptake capacity, permeability and stiffness. It was further tested whether the 3D cultivation permits the cells to be sent more convenient and to be cultivated over 10 days without loss of viability. Hence, the effect of the scaffold cultivation on important metabolic functions of Primary human hepatocytes was tested for up to 10 days and compared to conventional 2D cultures. The basal urea production, the one of important metabolic functions of Primary human hepatocytes that cultured on optimaix-3D scaffolds was about $50 \%$ higher than Primary human hepatocytes cultured in 2D. Hence, basic urea production of Primary human hepatocytes in $2 \mathrm{D}$ cultures decreased quickly within 10 days. In contrast, the basic urea production in $3 \mathrm{D}$ cultures remained stable over 10 days. According to the results obtained from albumin synthesis tests, albumin synthesis in 
optimaix-3D cultures enhanced over the time compared to 2D cultures (Ruoß et al., 2018).

It is evident that vitrification has advantages over traditional slow freezing methods as a means of cryopreservation of mammalian ova and embryos. The most remarkable characteristic of vitrification is the prevention of ice crystal formation during the cooling process (Campos-Chillòn et al., 2006; Taylor-Robinson et al., 2014). Also, this procedure is less time-consuming and relatively inexpensive compared to conventional freezing protocols (Vajta et al., 1998). Although significant efforts have been made to appoint a novel vitrification procedure, large-scale application of IVF bovine embryos remains a relatively unexplored area of research. Evidently, vitrification offers significant potential applications for IVF and cloned bovine embryos in the field (Do et al., 2014).

\section{Conclusion:}

According to study of all of these kinds of cell shipping methods, we reach some conclusion about them. Based on the evaluation of appropriate time for cells transporting in these methods it is apparent that Cells preserved in the formula containing trehalose represented $70 \%$ viability for 3 weeks. After that Optimaix-3D Scaffold permits the cells to be sent more conveniently and to be cultivated over 10 days without loss of viability. Following that the density of cells sealed on Fibrin microbeads at room temperature did not alter even after 10 days at room temperature. After 6 day storage of cells on Fibrin microbeads at room temperature, no alteration of $\mathrm{pH}$ and gas level were detected in the medium. The appropriate storage duration for stem cells encapsulated within alginate gels is about five days under ambient conditions in an air-tight environment. The appropriate time for cells transporting by agarose-medium gel based method was 3 days (no more than 5 days). After cells were exposed in hypertonic betaine medium, it was able to significantly improve the viability of cells for 3 days and they could still attach to cell culture substrates. Altogether, we concluded that the preservation of cells by formula containing trehalose is much more convenient with the higher appropriate storage duration compared to other methods which were mentioned in this review. Although it should mentioned that the appropriate method for cell shipping is cell line dependent and it must be examined which method is the best one for different investigations.

\section{Abbreviations}

DMSO: dimethyl sulfoxide; CPAs: cryoprotective agents; PG: propylene glycol; EG: ethylene glycol; GLY: glycerol; GMP: good manufacturing practice; LN2: liquid nitrogen; Me2SO: dimethyl sulfoxide; hMSC: human mesenchymal stem cells; SEM: scanning electron microscope; HA: HEMA agarose; hESCs: human embryonic stem cells; hMSC: human mesenchymal stem cell; mESC: mouse embryonic stem cells; FMB: fibrin microbeads ; HIF-1: hypoxia induced factor-1; ECM: extracellular matrix; IVF: In vitro fertilization

\section{Declarations:}

\section{Acknowledgements}

Not applicable.

\section{Authors' contributions}

SHH and MH designed and drafted the manuscript, collected the references and carried out the primary literature search. SB and SKH modify the manuscript and participated in discussions. All authors read and approved the final manuscript.

\section{Funding}

Not applicable.

\section{Availability of data and materials}

Not applicable.

Ethics approval and consent to participate 
This is not applicable for this review.

\section{Consent for publication}

This is not applicable for this review.

\section{Competing interests}

The authors declare that they have no competing interests.

\section{REFERENCES:}

Armitage, W. J. (1987). Cryopreservation of animal cells. Symposia of the Society for Experimental Biology , 41, 379-393.

Azambuja, R. M., Kraemer, D. C., \& Westhusin, M. E. (1998). Effect of low temperatures on in-vitro matured bovine oocytes. Theriogenology , 49 (6), 1155-1164.

Baiocchini, A., Montaldo, C., Conigliaro, A., Grimaldi, A., Correani, V., Mura, F., Ciccosanti, F., Rotiroti, N., Brenna, A., \& Montalbano, M. (2016). Extracellular matrix molecular remodeling in human liver fibrosis evolution. PloS One, 11 (3), e0151736.

Bakhshpour, M., Idil, N., Percin, I., \& Denizli, A. (2019). Biomedical applications of polymeric cryogels. Applied Sciences ,9 (3), 553.

Barbaric, I., Jones, M., Buchner, K., Baker, D., Andrews, P. W., \& Moore, H. D. (2011). Pinacidil enhances survival of cryopreserved human embryonic stem cells. Cryobiology , 63 (3), 298-305.

Barun, S. (2015). A review on applications \& advantages of cryopreservation in different fields of science. The Beats Nat Sci, 2 , 1-6.

Baust, J. M., Van Buskirk, R., \& Baust, J. G. (2003).Normothermic, hypothermic and cryopreservation maintenance and storage of cells, tissues and organs in gel-based media . Google Patents.

Beattie, G. M., Crowe, J. H., Lopez, A. D., Cirulli, V., Ricordi, C., \& Hayek, A. (1997). Trehalose: a cryoprotectant that enhances recovery and preserves function of human pancreatic islets after long-term storage.Diabetes , 46 (3), 519-523.

Ben-Ari, A., Rivkin, R., Frishman, M., Gaberman, E., Levdansky, L., \& Gorodetsky, R. (2009). Isolation and implantation of bone marrow-derived mesenchymal stem cells with fibrin micro beads to repair a critical-size bone defect in mice. Tissue Engineering Part A ,15 (9), 2537-2546.

Bishop, A. L., \& Hall, A. (2000). Rho GTPases and their effector proteins. Biochemical Journal , 348 (2), $241-255$.

Bissoyi, A., Nayak, B., Pramanik, K., \& Sarangi, S. K. (2014). Targeting cryopreservation-induced cell death: a review.Biopreservation and Biobanking, 12 (1), 23-34.

Brown, I. R. (1990). Induction of heat shock (stress) genes in the mammalian brain by hyperthermia and other traumatic events: a current perspective. Journal of Neuroscience Research , 27 (3), 247-255.

Campos-Chillon, L. F., Walker, D. J., De La Torre-Sanchez, J. F., \& Seidel Jr, G. E. (2006). In vitro assessment of a direct transfer vitrification procedure for bovine embryos. Theriogenology ,65 (6), 12001214 .

Chakrabarti, R., \& Schutt, C. E. (2001). The enhancement of PCR amplification by low molecular-weight sulfones. Gene, 274 (1-2), 293-298.

Chatterjee, S., \& Gagnon, C. (2001). Production of reactive oxygen species by spermatozoa undergoing cooling, freezing, and thawing. Molecular Reproduction and Development: Incorporating Gamete Research , 59 (4), 451-458. 
Chen, B., Wright, B., Sahoo, R., \& Connon, C. J. (2013). A novel alternative to cryopreservation for the short-term storage of stem cells for use in cell therapy using alginate encapsulation. In Tissue Engineering - Part C: Methods (Vol. 19, Issue 7). https://doi.org/10.1089/ten.tec.2012.0489

Chen, K. L., Mylon, S. E., \& Elimelech, M. (2007). Enhanced aggregation of alginate-coated iron oxide (hematite) nanoparticles in the presence of calcium, strontium, and barium cations. Langmuir ,23 (11), 5920-5928.

Chen, T., Acker, J. P., Eroglu, A., Cheley, S., Bayley, H., Fowler, A., \& Toner, M. (2001). Beneficial effect of intracellular trehalose on the membrane integrity of dried mammalian cells. Cryobiology ,43 (2), 168-181.

Chen, Y., Foote, R. H., \& Brockett, C. C. (1993). Effect of sucrose, trehalose, hypotaurine, taurine, and blood serum on survival of frozen bull sperm. Cryobiology , 30 (4), 423-431.

Choi, Y. H., \& Chang, Y. J. (2003). The influence of cooling rate, developmental stage, and the addition of sugar on the cryopreservation of larvae of the pearl oyster Pinctada fucata martensii.Cryobiology , 46 (2), 190-193.

Choudhery, M. S., Badowski, M., Muise, A., \& Harris, D. T. (2015). Effect of mild heat stress on the proliferative and differentiative ability of human mesenchymal stromal cells. Cytotherapy ,17 (4), 359-368.

Chung, H. J., \& Park, T. G. (2009). Injectable cellular aggregates prepared from biodegradable porous microspheres for adipose tissue engineering. Tissue Engineering Part A , 15 (6), 1391-1400.

Cobo, F., Stacey, G. N., Hunt, C., Cabrera, C., Nieto, A., Montes, R., Cortes, J. L., Catalina, P., Barnie, A., \& Concha, A. (2005). Microbiological control in stem cell banks: Approaches to standardisation. Applied Microbiology and Biotechnology ,68 (4), 456-466. https://doi.org/10.1007/s00253-005-0062-2

Coopman, K. (2013). Alternatives to cryopreservation for the short and long-term storage of mammalian cells .

Courtenay, E. S., Capp, M. W., Anderson, C. F., \& Record, M. T. (2000). Vapor pressure osmometry studies of osmolyte- protein interactions: implications for the action of osmoprotectants in vivo and for the interpretation of "osmotic stress" experiments in vitro.Biochemistry , 39 (15), 4455-4471.

Criado, E., Albani, E., Novara, P. V., Smeraldi, A., Cesana, A., Parini, V., \& Levi-Setti, P. E. (2011). Human oocyte ultravitrification with a low concentration of cryoprotectants by ultrafast cooling: a new protocol. Fertility and Sterility, 95 (3), 1101-1103.

Crowe, J. H., \& Crowe, L. M. (2000). Preservation of mammalian cells-learning nature's tricks. Nature Biotechnology ,18 (2), 145-146.

Crowe, J. H., Crowe, L. M., Wolkers, W. F., Oliver, A. E., Ma, X., Auh, J.-H., Tang, M., Zhu, S., Norris, J., \& Tablin, F. (2005). Stabilization of dry mammalian cells: lessons from nature.Integrative and Comparative Biology , 45 (5), 810-820.

Crowe, J. H., Tablin, F., Wolkers, W. F., Gousset, K., Tsvetkova, N. M., \& Ricker, J. (2003). Stabilization of membranes in human platelets freeze-dried with trehalose. Chemistry and Physics of Lipids ,122 (1-2), $41-52$.

Daei-farshbaf, N., Ardeshirylajimi, A., Seyedjafari, E., Piryaei, A., Fathabady, F. F., Hedayati, M., Salehi, M., Soleimani, M., Nazarian, H., \& Moradi, S.-L. (2014). Bioceramic-collagen scaffolds loaded with human adipose-tissue derived stem cells for bone tissue engineering. Molecular Biology Reports , 41 (2), 741-749.

De Vries, E. G. E., Vellenga, E., Kluin-Nelemans, J. C., \& Mulder, N. H. (2004). The happy destiny of frozen haematopoietic stem cells: from immature stem cells to mature applications. European Journal of Cancer , 40 (13), 1987-1992. 
Di, G., Wang, J., Liu, M., Wu, C., Han, Y., \& Duan, H. (2012). Development and evaluation of a trehalosecontained solution formula to preserve hUC-MSCs at 4deg C. Journal of Cellular Physiology ,227 (3), 879-884.

Do, V. H., Walton, S., \& Taylor-Robinson, A. (2014). Benefits and constraints of vitrification technologies for cryopreservation of bovine in vitro fertilized embryos .

Ebertz, S. L., \& McGann, L. E. (2004). Cryoinjury in endothelial cell monolayers. Cryobiology , 49 (1), 37-44. https://doi.org/10.1016/j.cryobiol.2004.04.003

Ehrenreich, M., \& Ruszczak, Z. (2006). Update on tissue-engineered biological dressings. Tissue Engineering , 12 (9), 2407-2424. https://doi.org/10.1089/ten.2006.12.2407

El Assal, R., Abou-Elkacem, L., Tocchio, A., Pasley, S., Matosevic, S., Kaplan, D. L., Zylberberg, C., \& Demirci, U. (2019). Bioinspired preservation of natural killer cells for cancer immunotherapy.Advanced Science, 6 (6), 1802045.

El Assal, R., Guven, S., Gurkan, U. A., Gozen, I., Shafiee, H., Dalbeyler, S., Abdalla, N., Thomas, G., Fuld, W., \& Illigens, B. M. W. (2014). Bio-inspired cryo-ink preserves red blood cell phenotype and function during nanoliter vitrification. Advanced Materials ,26 (33), 5815-5822.

Erdag, G., Eroglu, A., Morgan, J. R., \& Toner, M. (2002). Cryopreservation of fetal skin is improved by extracellular trehalose. Cryobiology , 44 (3), 218-228.

Eroglu, A., Russo, M. J., Bieganski, R., Fowler, A., Cheley, S., Bayley, H., \& Toner, M. (2000). Intracellular trehalose improves the survival of cryopreserved mammalian cells. Nature Biotechnology ,18 (2), 163-167.

Eroglu, A., Toner, M., \& Toth, T. L. (2002). Beneficial effect of microinjected trehalose on the cryosurvival of human oocytes.Fertility and Sterility, 77 (1), 152-158.

Esfandiarei, M., Boroomand, S., Suarez, A., Si, X., Rahmani, M., \& McManus, B. (2007). Coxsackievirus B3 activates nuclear factor kappa B transcription factor via a phosphatidylinositol-3 kinase/protein kinase B-dependent pathway to improve host cell viability. Cellular Microbiology , 9 (10), 2358-2371.

Fahy, G. M., Wowk, B., Wu, J., \& Paynter, S. (2004). Improved vitrification solutions based on the predictability of vitrification solution toxicity. Cryobiology , 48 (1), 22-35.

Fan, W.-X., Ma, X.-H., Ge, D., Liu, T.-Q., \& Cui, Z.-F. (2009). Cryoprotectants for the vitrification of corneal endothelial cells. Cryobiology , 58 (1), 28-36.

Fowke, K. R., Behnke, J., Hanson, C., Shea, K., \& Cosentino, L. M. (2000). Apoptosis: a method for evaluating the cryopreservation of whole blood and peripheral blood mononuclear cells. Journal of Immunological Methods, 244 (1-2), 139-144.

Fujioka, T., Yasuchika, K., Nakamura, Y., Nakatsuji, N., \& Suemori, H. (2004). A simple and efficient cryopreservation method for primate embryonic stem cells. International Journal of Developmental Biology , $48(10), 1149-1154$.

Fuller, B. J., Petrenko, A. Y., Rodriguez, J. V, Somov, A. Y., Balaban, C. L., \& Guibert, E. E. (2016). Biopreservation of hepatocytes: current concepts on hypothermic preservation, cryopreservation, and vitrification. CryoLetters , 37 (4), 432-452.

Galluzzi, L., Vitale, I., Aaronson, S. A., Abrams, J. M., Adam, D., Agostinis, P., Alnemri, E. S., Altucci, L., Amelio, I., \& Andrews, D. W. (2018). Molecular mechanisms of cell death: recommendations of the Nomenclature Committee on Cell Death 2018. Cell Death 83 Differentiation , 25 (3), 486-541.

Gao, D., \& Critser, J. K. (2000). Mechanisms of cryoinjury in living cells. ILAR Journal , 41 (4), 187-196. https://doi.org/10.1093/ilar.41.4.187 
Gao, H.-H., Li, Z.-P., Wang, H.-P., Zhang, L.-F., \& Zhang, J.-M. (2016). Cryopreservation of whole bovine ovaries: comparisons of different thawing protocols. European Journal of Obstetrics 83 Gynecology and Reproductive Biology , 204 , 104-107.

Gorodetsky, R., An, J., Levdansky, L., Vexler, A., Berman, E., Clark, R. A. F., Gailit, J., \& Marx, G. (1999). Fibrin microbeads (FMB) as biodegradable carriers for culturing cells and for accelerating wound healing. Journal of Investigative Dermatology , 112 (6), 866-872.

Gorodetsky, R., Levdansky, L., Gaberman, E., Gurevitch, O., Lubzens, E., \& McBride, W. H. (2011). Fibrin microbeads loaded with mesenchymal cells support their long-term survival while sealed at room temperature.Tissue Engineering - Part C: Methods , 17 (7), 745-755. https://doi.org/10.1089/ten.tec.2010.0644

Grein, T. A., Freimark, D., Weber, C., Hudel, K., Wallrapp, C., \& Czermak, P. (2010). Alternatives to dimethylsulfoxide for serum-free cryopreservation of human mesenchymal stem cells. International Journal of Artificial Organs , 33 (6), 370-380. https://doi.org/10.1177/039139881003300605

Hanna, J., \& Hubel, A. (2009). Preservation of stem cells.Organogenesis , 5 (3), 134-137. https://doi.org/10.4161/org.5.3.9585

Hara, J., Tottori, J., Anders, M., Dadhwal, S., Asuri, P., \& Mobed-Miremadi, M. (2017). Trehalose effectiveness as a cryoprotectant in 2D and 3D cell cultures of human embryonic kidney cells.Artificial Cells, Nanomedicine, and Biotechnology , 45 (3), 609-616.

He, X., Park, E. Y. H., Fowler, A., Yarmush, M. L., \& Toner, M. (2008). Vitrification by ultra-fast cooling at a low concentration of cryoprotectants in a quartz micro-capillary: a study using murine embryonic stem cells. Cryobiology , 56 (3), 223-232.

Hegner, B., Weber, M., Dragun, D., \& Schulze-Lohoff, E. (2005). Differential regulation of smooth muscle markers in human bone marrow-derived mesenchymal stem cells. Journal of Hypertension ,23 (6), 11911202 .

Heng, B. C., Clement, M. V., \& Cao, T. (2007). Caspase inhibitor Z-VAD-FMK enhances the freeze-thaw survival rate of human embryonic stem cells. Bioscience Reports , 27 (4-5), 257-264.

Heng, B. C., Ye, C. P., Liu, H., Toh, W. S., Rufaihah, A. J., Yang, Z., Bay, B. H., Ge, Z., Ouyang, H. W., \& Lee, E. H. (2006). Loss of viability during freeze-thaw of intact and adherent human embryonic stem cells with conventional slow-cooling protocols is predominantly due to apoptosis rather than cellular necrosis. Journal of Biomedical Science , 13 (3), 433-445.

Heo, Y. S., Nagrath, S., Moore, A. L., Zeinali, M., Irimia, D., Stott, S. L., Toth, T. L., \& Toner, M. (2015). "Universal" vitrification of cells by ultra-fast cooling. Technology, 3 (01), 64-71.

Hopkins, J. B., Badeau, R., Warkentin, M., \& Thorne, R. E. (2012). Effect of common cryoprotectants on critical warming rates and ice formation in aqueous solutions. Cryobiology , 65 (3), 169-178.

Hunt, C. J. (2007). The banking and cryopreservation of human embryonic stem cells. Transfusion Medicine and Hemotherapy , 34 (4), 293-304.

Jain, M., Rajan, R., Hyon, S.-H., \& Matsumura, K. (2014). Hydrogelation of dextran-based polyampholytes with cryoprotective properties via click chemistry. Biomaterials Science, 2 (3), 308-317.

Jang, T. H., Park, S. C., Yang, J. H., Kim, J. Y., Seok, J. H., Park, U. S., Choi, C. W., Lee, S. R., \& Han, J. (2017). Cryopreservation and its clinical applications. Integrative Medicine Research ,6 (1), 12-18.

Janz, F. de L., Debes, A. de A., Cavaglieri, R. de C., Duarte, S. A., Romao, C. M., Moron, A. F., Zugaib, M., \& Bydlowski, S. P. (2012). Evaluation of distinct freezing methods and cryoprotectants for human amniotic fluid stem cells cryopreservation. Journal of Biomedicine and Biotechnology, 2012 . 
Jenkins, E. C., Ye, L., \& Silverman, W. P. (2012). Does the cryogenic freezing process cause shorter telomeres? Cryobiology ,65 (1), 72-73.

Ji, L., de Pablo, J. J., \& Palecek, S. P. (2004). Cryopreservation of adherent human embryonic stem cells. Biotechnology and Bioengineering , 88 (3), 299-312.

Jin, B., Kusanagi, K., Ueda, M., Seki, S., Valdez Jr, D. M., Edashige, K., \& Kasai, M. (2008). Formation of extracellular and intracellular ice during warming of vitrified mouse morulae and its effect on embryo survival. Cryobiology , 56 (3), 233-240.

Kang, S.-W., Seo, S.-W., Choi, C. Y., \& Kim, B.-S. (2008). Porous poly (lactic-co-glycolic acid) microsphere as cell culture substrate and cell transplantation vehicle for adipose tissue engineering. Tissue Engineering Part C: Methods , 14 (1), 25-34.

Karlsson, J. O. M. M., \& Toner, M. (1996). Long-term storage of tissues by cryopreservation: critical issues. Biomaterials , 17 (3), 243-256. https://doi.org/10.1016/0142-9612(96)85562-1

Katenz, E., Vondran, F. W. R., Schwartlander, R., Pless, G., Gong, X., Cheng, X., Neuhaus, P., \& Sauer, I. M. (2007). Cryopreservation of primary human hepatocytes: the benefit of trehalose as an additional cryoprotective agent. Liver Transplantation , 13 (1), 38-45.

Katsen-Globa, A., Meiser, I., Petrenko, Y. A., Ivanov, R. V, Lozinsky, V. I., Zimmermann, H., \& Petrenko, A. Y. (2014). Towards ready-to-use 3-D scaffolds for regenerative medicine: adhesion-based cryopreservation of human mesenchymal stem cells attached and spread within alginate-gelatin cryogel scaffolds. Journal of Materials Science: Materials in Medicine, 25 (3), 857-871.

Kaushik, S., \& Kaur, J. (2005). Effect of chronic cold stress on intestinal epithelial cell proliferation and inflammation in rats.Stress , 8 (3), 191-197.

Khan, R., \& Khan, M. H. (2013). Use of collagen as a biomaterial: An update. Journal of Indian Society of Periodontology ,17 (4), 539.

Kim, S. J., Park, J. H., Lee, J. E., Kim, J. M., Lee, J. B., Moon, S. Y., Roh, S. Il, Kim, C. G., \& Yoon, H. S. (2004). Effects of type IV collagen and laminin on the cryopreservation of human embryonic stem cells. Stem Cells , 22 (6), 950-961.

Kumari, J., \& Kumar, A. (2017). Development of polymer based cryogel matrix for transportation and storage of mammalian cells.Scientific Reports , 7 (January), 1-13. https://doi.org/10.1038/srep41551

Kuwayama, M., Vajta, G., Kato, O., \& Leibo, S. P. (2005). Highly efficient vitrification method for cryopreservation of human oocytes.Reproductive Biomedicine Online , 11 (3), 300-308.

Lawson, A., Ahmad, H., \& Sambanis, A. (2011). Cytotoxicity effects of cryoprotectants as single-component and cocktail vitrification solutions. Cryobiology ,62 (2), 115-122.

Lee, C. H., Singla, A., \& Lee, Y. (2001). Biomedical applications of collagen. International Journal of Pharmaceutics ,221 (1-2), 1-22.

Lee, H.-J., Elmoazzen, H., Wright, D. L. L., Biggers, J. L., Toner, M., \& Toth, T. L. (2009). Ultra-rapid vitrification of oocytes in low cryoprotectant concentration using quart capillary. Fertility and Sterility, 92 (3), S71.

Lee, K.-H., Sun, J.-C., Chuang, C., Guo, S.-F., Tu, C.-F., \& Ju, J.-C. (2013). An efficient and mass reproducible method for vitrifying mouse embryos on a paper in cryotubes. Cryobiology , 66 (3), 311-317.

Lee, Y.-A., Kim, Y.-H., Kim, B.-J., Kim, B.-G., Kim, K.-J., Auh, J.-H., Schmidt, J. A., \& Ryu, B.-Y. (2013). Cryopreservation in trehalose preserves functional capacity of murine spermatogonial stem cells. PloS One, 8 (1), e54889. 
Li, A. P. (2007). Human hepatocytes: isolation, cryopreservation and applications in drug development. Chemico-Biological Interactions, 168 (1), 16-29.

Li, Y., Si, W., Zhang, X., Dinnyes, A., \& Ji, W. (2003). Effect of amino acids on cryopreservation of cynomolgus monkey (Macaca fascicularis) sperm. American Journal of Primatology: Official Journal of the American Society of Primatologists , 59 (4), 159-165.

Linnebacher, M., Maletzki, C., Ostwald, C., Klier, U., Krohn, M., Klar, E., \& Prall, F. (2010). Cryopreservation of human colorectal carcinomas prior to xenografting. BMC Cancer , 10 (1), 362.

Malpique, R., Osorio, L. M., Ferreira, D. S., Ehrhart, F., Brito, C., Zimmermann, H., \& Alves, P. M. (2010). Alginate encapsulation as a novel strategy for the cryopreservation of neurospheres. Tissue Engineering Part C: Methods , 16 (5), 965-977.

Mandawala, A. A., Harvey, S. C., Roy, T. K., \& Fowler, K. E. (2016). Cryopreservation of animal oocytes and embryos: Current progress and future prospects. Theriogenology, 86 (7), 1637-1644.

Mantel, N., Caruso, T. D., Kazam, E., Stevenson, R. E., \& Flandermeyer, R. R. (1979). [3] preservation, storage, and shipment 29.LVIII , 29-36.

Martinetti, D., Colarossi, C., Buccheri, S., Denti, G., Memeo, L., \& Vicari, L. (2017). Effect of trehalose on cryopreservation of pure peripheral blood stem cells. Biomedical Reports , 6 (3), 314-318.

Martinsen, A., Skjak-Braek, G., \& Smidsrod, O. (1989). Alginate as immobilization material: I. Correlation between chemical and physical properties of alginate gel beads. Biotechnology and Bioengineering , 33 (1), 79-89.

Mazur, P. (2004). Life in the frozen state. In Principles of Cryobiology. CRC Press, Boca Raton, FL, USA. Academic Press. Boca Raton(Boca Raton). Boca Raton, FL: CRC Press.

Mazur, Peter. (1970). Cryobiology: the freezing of biological systems.Science, 168 (3934), 939-949.

Mazur, Peter, \& Seki, S. (2011). Survival of mouse oocytes after being cooled in a vitrification solution to$196 \mathrm{C}$ at 95 to $70,000 \mathrm{C} / \mathrm{min}$ and warmed at 610 to $118,000 \mathrm{C} / \mathrm{min}$ : A new paradigm for cryopreservation by vitrification. Cryobiology, 62 (1), 1-7.

McGann, L. E. (1978). Differing actions of penetrating and nonpenetrating cryoprotective agents. Cryobiology , 15 (4), 382-390.

Melino, G. (2011). p63 is a suppressor of tumorigenesis and metastasis interacting with mutant p53. Cell Death 8 Differentiation ,18 (9), 1487-1499.

Meryman, H. T. (2007). Cryopreservation of living cells: principles and practice. Transfusion , 47 (5), 935-945.

Messmer, M. N., Snyder, A. G., \& Oberst, A. (2019). Comparing the effects of different cell death programs in tumor progression and immunotherapy. Cell Death $\&$ Differentiation , 26 (1), 115-129.

Miyamoto, Y., Enosawa, S., Takeuchi, T., \& Takezawa, T. (2009). Cryopreservation in situ of cell monolayers on collagen vitrigel membrane culture substrata: ready-to-use preparation of primary hepatocytes and ES cells. Cell Transplantation, 18 (5-6), 619-626.

Miyamoto, Y., Ikeuchi, M., Noguchi, H., \& Hayashi, S. (2018). Long-term Cryopreservation of Human and other Mammalian Cells at- 80deg C for 8 Years. Cell Medicine, 10 , 2155179017733148.

Miyamoto, Y., Noguchi, H., Yukawa, H., Oishi, K., Matsushita, K., Iwata, H., \& Hayashi, S. (2012). Cryopreservation of induced pluripotent stem cells. Cell Medicine, 3 (1-3), 89-95.

Miyamoto, Y., Oishi, K., Yukawa, H., Noguchi, H., Sasaki, M., Iwata, H., \& Hayashi, S. (2012). Cryopreservation of human adipose tissue-derived stem/progenitor cells using the silk protein sericin. Cell 
Transplantation , 21 (2-3), 617-622.

Miyamoto, Y., Suzuki, S., Nomura, K., \& Enosawa, S. (2006). Improvement of hepatocyte viability after cryopreservation by supplementation of long-chain oligosaccharide in the freezing medium in rats and humans.Cell Transplantation, 15 (10), 911-919.

Morch, Y. A., Donati, I., Strand, B. L., \& Skjak-Bræk, G. (2006). Effect of Ca2+, Ba2+, and Sr2+ on alginate microbeads.Biomacromolecules , 7 (5), 1471-1480.

Morris, G. J. (2005). The origin, ultrastructure, and microbiology of the sediment accumulating in liquid nitrogen storage vessels. Cryobiology , 50 (3), 231-238.

Morris, G. J., Goodrich, M., Acton, E., \& Fonseca, F. (2006). The high viscosity encountered during freezing in glycerol solutions: effects on cryopreservation. Cryobiology , 52 (3), 323-334.

Motta, J. P. R., Paraguassú-Braga, F. H., Bouzas, L. F., \& Porto, L. C. (2014). Evaluation of intracellular and extracellular trehalose as a cryoprotectant of stem cells obtained from umbilical cord blood. Cryobiology , 68 (3), 343-348.

Moussa, M., Dumont, F., Perrier-Cornet, J., \& Gervais, P. (2008). Cell inactivation and membrane damage after long-term treatments at sub-zero temperature in the supercooled and frozen states. Biotechnology and Bioengineering , 101 (6), 1245-1255.

Nazio, F., Bordi, M., Cianfanelli, V., Locatelli, F., \& Cecconi, F. (2019). Autophagy and cancer stem cells: molecular mechanisms and therapeutic applications. Cell Death $\&$ Differentiation ,26 (4), 690-702.

Nema, R., \& Khare, S. (2012). An animal cell culture: Advance technology for modern research. Advances in Bioscience and Biotechnology , 03 (03), 219-226. https://doi.org/10.4236/abb.2012.33030

Nomura, M., Muramoto, Y., Yasuda, S., Takabe, T., \& Kishitani, S. (1995). The accumulation of glycinebetaine during cold acclimation in early and late cultivars of barley. Euphytica , 83 (3), 247-250.

Omori, K., Valiente, L., Orr, C., Rawson, J., Ferreri, K., Todorov, I., Al-Abdullah, I. H., Medicherla, S., Potter, A. A., \& Schreiner, G. F. (2007). Improvement of human islet cryopreservation by a p38 MAPK inhibitor. American Journal of Transplantation, 7 (5), 1224-1232.

Park, J. S., Yang, H. N., Woo, D. G., Jeon, S. Y., \& Park, K.-H. (2011). The promotion of chondrogenesis, osteogenesis, and adipogenesis of human mesenchymal stem cells by multiple growth factors incorporated into nanosphere-coated microspheres. Biomaterials , 32 (1), 28-38.

Pi, C.-H., Hornberger, K., Dosa, P., \& Hubel, A. (2020). Understanding the freezing responses of T cells and other subsets of human peripheral blood mononuclear cells using DSMO-free cryoprotectants. Cytotherapy .

Pi, C.-H., Yu, G., Petersen, A., \& Hubel, A. (2018). Characterizing the "sweet spot" for the preservation of a T-cell line using osmolytes.Scientific Reports , 8 (1), 1-13.

Pi, C., Yu, G., Dosa, P. I., \& Hubel, A. (2019). Characterizing modes of action and interaction for multicomponent osmolyte solutions on Jurkat cells. Biotechnology and Bioengineering , 116 (3), 631-643.

Place, E. S., Rojo, L., Gentleman, E., Sardinha, J. P., \& Stevens, M. M. (2011). Strontium-and zinc-alginate hydrogels for bone tissue engineering. Tissue Engineering Part A , 17 (21-22), 2713-2722.

Prento, P. (1997). The effects of freezing, storage, and thawing on cell compartment integrity and ultrastructure. Histochemistry and Cell Biology, 108 (6), 543-547.

Pu, L. L. Q., Cui, X., Fink, B. F., Cibull, M. L., \& Gao, D. (2005). Cryopreservation of adipose tissues: the role of trehalose.Aesthetic Surgery Journal , 25 (2), 126-131.

Ranucci, C. S., Kumar, A., Batra, S. P., \& Moghe, P. V. (2000). Control of hepatocyte function on collagen foams: sizing matrix pores toward selective induction of 2-D and 3-D cellular morphogenesis.Biomaterials , 
21 (8), 783-793.

Reddig, P. J., \& Juliano, R. L. (2005). Clinging to life: cell to matrix adhesion and cell survival. Cancer and Metastasis Reviews ,24 (3), 425-439. https://doi.org/10.1007/s10555-005-5134-3

Reubinoff, B. E., Pera, M. F., Vajta, G., \& Trounson, A. O. (2001). Effective cryopreservation of human embryonic stem cells by the open pulled straw vitrification method. Human Reproduction ,16 (10), 21872194.

Rubinstein, P., Dobrila, L., Rosenfield, R. E., Adamson, J. W., Migliaccio, G., Migliaccio, A. R., Taylor, P. E., \& Stevens, C. E. (1995). Processing and cryopreservation of placental/umbilical cord blood for unrelated bone marrow reconstitution. Proceedings of the National Academy of Sciences , 92 (22), 10119-10122.

Rudolph, A. S., \& Crowe, J. H. (1985). Membrane stabilization during freezing: the role of two natural cryoprotectants, trehalose and proline. Cryobiology, 22 (4), 367-377.

Ruoss, M., Haussling, V., Schugner, F., Olde Damink, L. H. H., Lee, S. M. L., Ge, L., Ehnert, S., \& Nussler, A. K. (2018). A standardized collagen-based scaffold improves human hepatocyte shipment and allows metabolic studies over 10 days. Bioengineering , 5 (4), 86.

Sablina, A. A., Budanov, A. V, Ilyinskaya, G. V, Agapova, L. S., Kravchenko, J. E., \& Chumakov, P. M. (2005). The antioxidant function of the p53 tumor suppressor. Nature Medicine , 11 (12), 1306-1313.

Sambu, S. (2015). A Bayesian approach to optimizing cryopreservation protocols. PeerJ , 3 , e1039.

Sarkar, J., \& Kumar, A. (2016). Thermo-responsive polymer aided spheroid culture in cryogel based platform for high throughput drug screening. Analyst , 141 (8), 2553-2567.

Satpathy, G. R., Torok, Z., Bali, R., Dwyre, D. M., Little, E., Walker, N. J., Tablin, F., Crowe, J. H., \& Tsvetkova, N. M. (2004). Loading red blood cells with trehalose: a step towards biostabilization. Cryobiology , $49(2), 123-136$.

Scheinkonig, C., Kappicht, S., Kolb, H. J., \& Schleuning, M. (2004). Adoption of long-term cultures to evaluate the cryoprotective potential of trehalose for freezing hematopoietic stem cells. Bone Marrow Transplantation, 34 (6), 531-536.

Schoenhard, J. A., \& Hatzopoulos, A. K. (2010). Stem cell therapy: Pieces of the puzzle. Journal of Cardiovascular Translational Research , 3 (1), 49-60. https://doi.org/10.1007/s12265-009-9148-z

Schoof, H., Apel, J., Heschel, I., \& Rau, G. (2001). Control of pore structure and size in freeze-dried collagen sponges. Journal of Biomedical Materials Research: An Official Journal of The Society for Biomaterials, The Japanese Society for Biomaterials, and The Australian Society for Biomaterials and the Korean Society for Biomaterials ,58 (4), 352-357.

Scott, K. L., Lecak, J., \& Acker, J. P. (2005). Biopreservation of red blood cells: past, present, and future. Transfusion Medicine Reviews, 19 (2), 127-142.

Seo, J. M., Sohn, M. Y., Suh, J. S., Atala, A., Yoo, J. J., \& Shon, Y.-H. (2011). Cryopreservation of amniotic fluid-derived stem cells using natural cryoprotectants and low concentrations of dimethylsulfoxide. Cryobiology , 62 (3), 167-173.

Serra, M., Correia, C., Malpique, R., Brito, C., Jensen, J., Bjorquist, P., Carrondo, M. J. T., \& Alves, P. M. (2011). Microencapsulation technology: a powerful tool for integrating expansion and cryopreservation of human embryonic stem cells. PloS One ,6 (8), e23212.

Shanina, I. V, Kravchenko, L. P., Fuller, B. J., \& Grischenko, V. I. (2000). A comparison of a sucrose-based solution with other preservation media for cold storage of isolated hepatocytes. Cryobiology ,41 (4), 315-318.

Shao, Q., \& Jiang, S. (2015). Molecular understanding and design of zwitterionic materials. Advanced Materials, 27 (1), 15-26. 
Sharma, S. D. (2005). Cryopreservation of somatic embryos - An overview .

Shimony, N., Gorodetsky, R., Marx, G., Gal, D., Rivkin, R., Ben-Ari, A., Landsman, A., \& Haviv, Y. S. (2006). Fibrin microbeads (FMB) as a 3D platform for kidney gene and cell therapy. Kidney International ,69 (3), 625-633.

Stacey, G. N., Connon, C. J., Coopman, K., Dickson, A. J., Fuller, B., Hunt, C. J., Kemp, P., Kerby, J., Man, J., \& Matejtschuk, P. (2017). Preservation and stability of cell therapy products: recommendations from an expert workshop. Regenerative Medicine , 12 (5), 553-564.

Stacey, G. N., \& Masters, J. R. (2008). Cryopreservation and banking of mammalian cell lines. Nature Protocols , 3 (12), 1981.

Stefansson, S., Han, S., Jeon, Y. I., Chung, D. S., Hwang, P., Le, H., Warden, J. L., \& Ho, D. (2017). Transporting Mammalian Cells at Ambient Temperature: A Viable Alternative to Dry Ice. Advances in Bioscience and Biotechnology , 8 (4), 127-133.

Stevens, V. L., Patel, A. V., Feigelson, H. S., Rodriguez, C., Thun, M. J., \& Calle, E. E. (2007). Cryopreservation of whole blood samples collected in the field for a large epidemiologic study. Cancer Epidemiology Biomarkers and Prevention , 16 (10), 2160-2163. https://doi.org/10.1158/1055-9965.EPI-07-0604

Sundaramurthi, P., \& Suryanarayanan, R. (2012). Calorimetry and complementary techniques to characterize frozen and freeze-dried systems. Advanced Drug Delivery Reviews , 64 (5), 384-395.

Suuronen, E. J., Kuraitis, D., \& Ruel, M. (2008). Improving Cell Engraftment with Tissue Engineering. Seminars in Thoracic and Cardiovascular Surgery , 20 (2), 110-114. https://doi.org/10.1053/j.semtcvs.2008.03.005

Svalgaard, J. D., Talkhoncheh, M. S., Haastrup, E. K., Munthe-Fog, L., Clausen, C., Hansen, M. B., Andersen, P., Gorlov, J. S., Larsson, J., \& Fischer-Nielsen, A. (2018). Pentaisomaltose, an alternative to DMSO. Engraftment of cryopreserved human CD34+ cells in immunodeficient NSG mice. Cell Transplantation, 27 (9), 1407-1412.

Swioklo, S., Constantinescu, A., \& Connon, C. J. (2016). Alginate-encapsulation for the improved hypothermic preservation of human adipose-derived stem cells. Stem Cells Translational Medicine , 5 (3), 339-349.

Syme, R., Bewick, M., Stewart, D., Porter, K., Chadderton, T., \& Gluck, S. (2004). The role of depletion of dimethyl sulfoxide before autografting: on hematologic recovery, side effects, and toxicity.Biology of Blood and Marrow Transplantation, 10 (2), 135-141.

Taylor-Robinson, A. W., Walton, S., Swain, D. L., Walsh, K. B., \& Vajta, G. (2014). The potential for modification in cloning and vitrification technology to enhance genetic progress in beef cattle in Northern Australia. Animal Reproduction Science ,148 (3-4), 91-96.

Taylor, M. J., Weegman, B. P., Baicu, S. C., \& Giwa, S. E. (2019). New approaches to cryopreservation of cells, tissues, and organs. Transfusion Medicine and Hemotherapy , 46 (3), 197-215.

Theus, M. H., Wei, L., Cui, L., Francis, K., Hu, X., Keogh, C., \& Yu, S. P. (2008). In vitro hypoxic preconditioning of embryonic stem cells as a strategy of promoting cell survival and functional benefits after transplantation into the ischemic rat brain. Experimental Neurology , 210 (2), 656-670. https://doi.org/10.1016/j.expneurol.2007.12.020

Tounkara, F. K., Dumont, N., Fournier, S., Boyer, L., Nadeau, P., \& Pineault, N. (2012). Mild hyperthermia promotes and accelerates development and maturation of erythroid cells. Stem Cells and Development, 21 (17), 3197-3208.

Troitzsch, R. Z., Vass, H., Hossack, W. J., Martyna, G. J., \& Crain, J. (2008). Molecular mechanisms of cryoprotection in aqueous proline: light scattering and molecular dynamics simulations. The Journal of Physical Chemistry B , 112 (14), 4290-4297. 
Trusal, L. R., Guzman, A. W., \& Baker, C. J. (1984). Characterization of freeze-thaw induced ultrastructural damage to endothelial cells in vitro. In Vitro , 20 (4), 353-364.

Umemura, E., Yamada, Y., Nakamura, S., Ito, K., Hara, K., \& Ueda, M. (2011). Viable cryopreserving tissue-engineered cell-biomaterial for cell banking therapy in an effective cryoprotectant. Tissue Engineering Part C: Methods , 17 (8), 799-807.

Vajta, G., Holm, P., Kuwayama, M., Booth, P. J., Jacobsen, H., Greve, T., \& Callesen, H. (1998). Open pulled straw (OPS) vitrification: a new way to reduce cryoinjuries of bovine ova and embryos. Molecular Reproduction and Development: Incorporating Gamete Research ,51 (1), 53-58.

Vajta, G., \& Nagy, Z. P. (2006). Are programmable freezers still needed in the embryo laboratory? Review on vitrification. Reproductive Biomedicine Online , 12 (6), 779-796.

Vergara, M., Becerra, S., Berrios, J., Osses, N., Reyes, J., Rodriguez-Moya, M., Gonzalez, R., \& Altamirano, C. (2014). Differential effect of culture temperature and specific growth rate on $\mathrm{CHO}$ cell behavior in chemostat culture. PLoS One, 9 (4), e93865.

Vigneault, C., Thompson, J., Wu, S., Hui, K. P. C., \& LeBlanc, D. I. (2009). Transportation of fresh horticultural produce. Postharvest Technologies for Horticultural Crops , 2 (1), 1-24.

von Bomhard, A., Elsasser, A., Ritschl, L. M., Schwarz, S., \& Rotter, N. (2016). Cryopreservation of endothelial cells in various cryoprotective agents and media-vitrification versus slow freezing methods. PLoS One, 11 (2), e0149660.

Vrana, N. E., Matsumura, K., Hyon, S., Geever, L. M., Kennedy, J. E., Lyons, J. G., Higginbotham, C. L., Cahill, P. A., \& McGuinness, G. B. (2012). Cell encapsulation and cryostorage in PVA-gelatin cryogels: incorporation of carboxylated $\varepsilon$-poly-L-lysine as cryoprotectant.Journal of Tissue Engineering and Regenerative Medicine ,6 (4), 280-290.

Wang, Juan, Wei, Y., Zhao, S., Zhou, Y., He, W., Zhang, Y., \& Deng, W. (2017). The analysis of viability for mammalian cells treated at different temperatures and its application in cell shipment. PloS One, 12 (4), e0176120.

Wang, Junjian, Chen, P., Xu, J., Zou, J. X., Wang, H., \& Chen, H.-W. W. (2015). Transporting cells in semi-solid gel condition and at ambient temperature. PloS One , 10 (6), e0128229. https://doi.org/10.1371/journal.pone.0128229

Watanabe, K., Imanishi, S., Akiduki, G., Cornette, R., \& Okuda, T. (2016). Air-dried cells from the anhydrobiotic insect, Polypedilum vanderplanki, can survive long term preservation at room temperature and retain proliferation potential after rehydration. Cryobiology, 73 (1), 93-98.

Wheatley, S. P., \& Wheatley, D. N. (2019). Transporting cells over several days without dry-ice. Journal of Cell Science ,132 (21).

Wille, J. J., Burdge, J. J., \& Park, J. Y. (2014). Methods for the preparation of an autologous serum-free cultured epidermis and for autografting applications. In Epidermal Cells (pp. 203-218). Springer.

Wise, H., Abel, P. W., \& Cawkill, D. (2009). Use of reduced temperature cell pausing to enhance flexibility of cell-based assays. Journal of Biomolecular Screening , 14 (6), 716-722.

Wolkers, W. F., Tablin, F., \& Crowe, J. H. (2002). From anhydrobiosis to freeze-drying of eukaryotic cells. Comparative Biochemistry and Physiology Part A: Molecular 83 Integrative Physiology, 131 (3), 535-543.

Wolkers, W. F., Walker, N. J., Tablin, F., \& Crowe, J. H. (2001). Human platelets loaded with trehalose survive freeze-drying. Cryobiology , 42 (2), 79-87.

Woods, E. J., Pollok, K. E., Byers, M. A., Perry, B. C., Purtteman, J., Heimfeld, S., \& Gao, D. (2007). Cord blood stem cell cryopreservation. Transfusion Medicine and Hemotherapy , 34 (4), 276-285. 
Wright, B., Cave, R. A., Cook, J. P., Khutoryanskiy, V. V, Mi, S., Chen, B., Leyland, M., \& Connon, C. J. (2012). Enhanced viability of corneal epithelial cells for efficient transport/storage using a structurally modified calcium alginate hydrogel. Regenerative Medicine ,7 (3), 295-307.

Xu, X., Cowley, S., Flaim, C. J., James, W., Seymour, L., \& Cui, Z. (2010). The roles of apoptotic pathways in the low recovery rate after cryopreservation of dissociated human embryonic stem cells.Biotechnology Progress, 26 (3), 827-837.

Yang, J., Cai, N., Zhai, H., Zhang, J., Zhu, Y., \& Zhang, L. (2016). Natural zwitterionic betaine enables cells to survive ultrarapid cryopreservation. Scientific Reports , 6 , 37458.

Yang, J., Pan, C., Sui, X., Cai, N., Zhang, J., Zhu, Y., \& Zhang, L. (2017). The hypothermic preservation of mammalian cells with assembling extracellular-matrix-mimetic microparticles. Journal of Materials Chemistry B , 5 (8), 1535-1541.

Yang, L., Li, C., Chen, L., \& Li, Z. (2009). An agarose-gel based method for transporting cell lines. Current Chemical Genomics ,3, 50 .

Yao, T., \& Asayama, Y. (2017). Animal-cell culture media: History, characteristics, and current issues. Reproductive Medicine and Biology , 16 (2), 99-117. https://doi.org/10.1002/rmb2.12024

Yavin, S., \& Arav, A. (2007). Measurement of essential physical properties of vitrification solutions. Theriogenology, 67 (1), 81-89.

Yokomise, H., Inui, K., Wada, H., Hasegawa, S., Ohno, N., \& Hitomi, S. (1995). Reliable cryopreservation of trachea for one month in a new trehalose solution. The Journal of Thoracic and Cardiovascular Surgery , $110(2), 382-385$.

Yong, K. W., Safwani, W. K. Z. W., Xu, F., Zhang, X., Choi, J. R., Abas, W. A. B. W., Omar, S. Z., Azmi, M. A. N., Chua, K. H., \& Pingguan-Murphy, B. (2017). Assessment of tumourigenic potential in long-term cryopreserved human adipose-derived stem cells. Journal of Tissue Engineering and Regenerative Medicine , 11 (8), 2217-2226.

Yong, K. W., Wan Safwani, W. K. Z., Xu, F., Wan Abas, W. A. B., Choi, J. R., \& Pingguan-Murphy, B. (2015). Cryopreservation of human mesenchymal stem cells for clinical applications: current methods and challenges.Biopreservation and Biobanking , 13 (4), 231-239.

Yu, G., Li, R., \& Hubel, A. (2018). Interfacial interactions of sucrose during cryopreservation detected by raman spectroscopy. Langmuir ,35 (23), 7388-7395.

Zhang, X. B., Li, K., Yau, K. H., Tsang, K. S., Fok, T. F., Li, C. K., Lee, S. M., \& Yuen, P. M. P. (2003). Trehalose ameliorates the cryopreservation of cord blood in a preclinical system and increases the recovery of CFUs, long-term culture-initiating cells, and nonobese diabetic-SCID repopulating cells. Transfusion , 43 (2), 265-272.

Zhou, X. L., Al Naib, A., Sun, D.-W., \& Lonergan, P. (2010). Bovine oocyte vitrification using the Cryotop method: effect of cumulus cells and vitrification protocol on survival and subsequent development. Cryobiology , $61(1), 66-72$.

Zimmermann, H., Zimmermann, D., Reuss, R., Feilen, P. J., Manz, B., Katsen, A., Weber, M., Ihmig, F. R., Ehrhart, F., \& Gessner, P. (2005). Towards a medically approved technology for alginate-based microcapsules allowing long-term immunoisolated transplantation. Journal of Materials Science: Materials in Medicine, 16 (6), 491-501. 


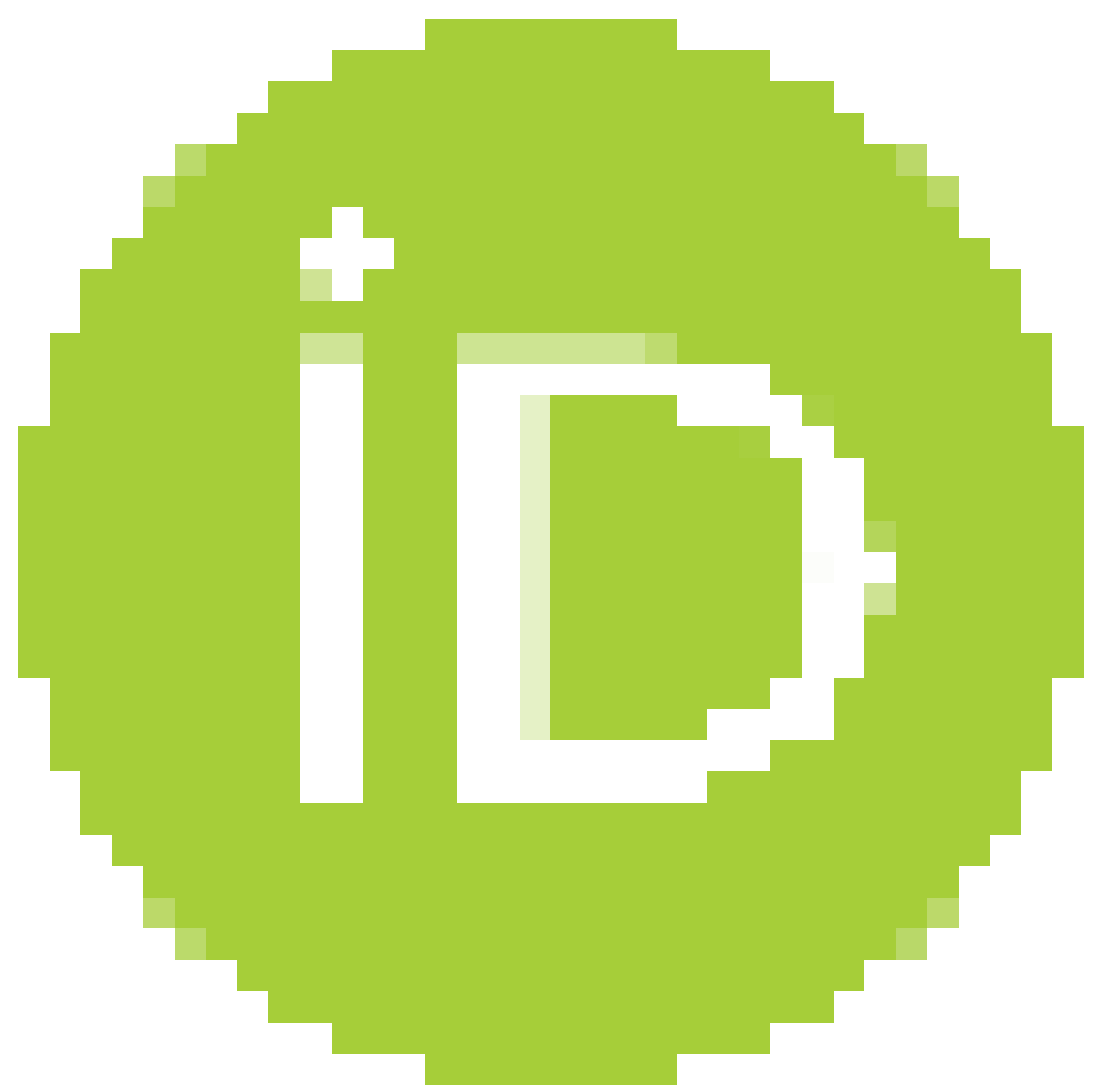

Table 1: Comparison between the slow-freezing and vitrification methods 


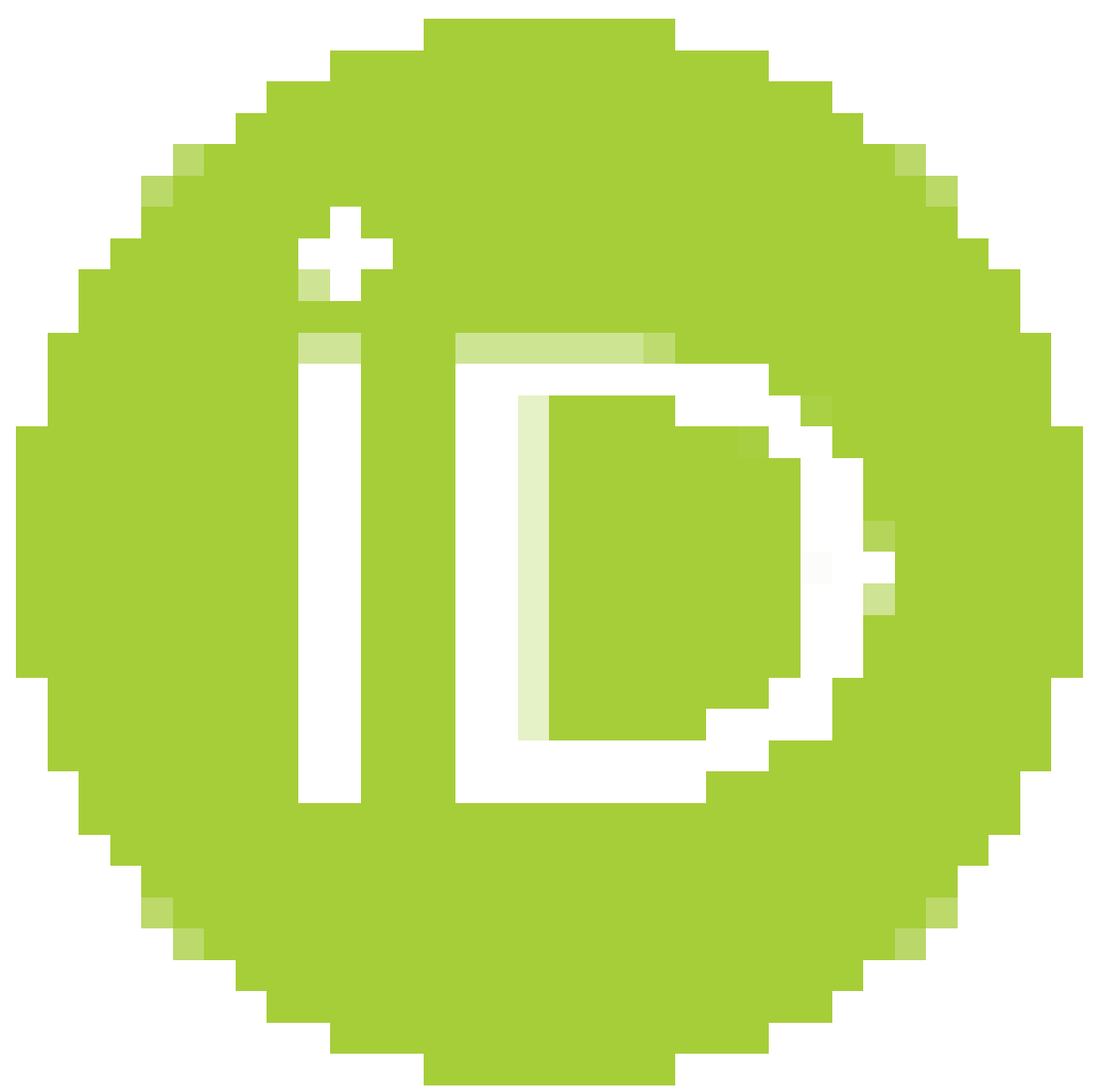

Table 2. Advantages and disadvantages of commonly used cryopreservation techniques 


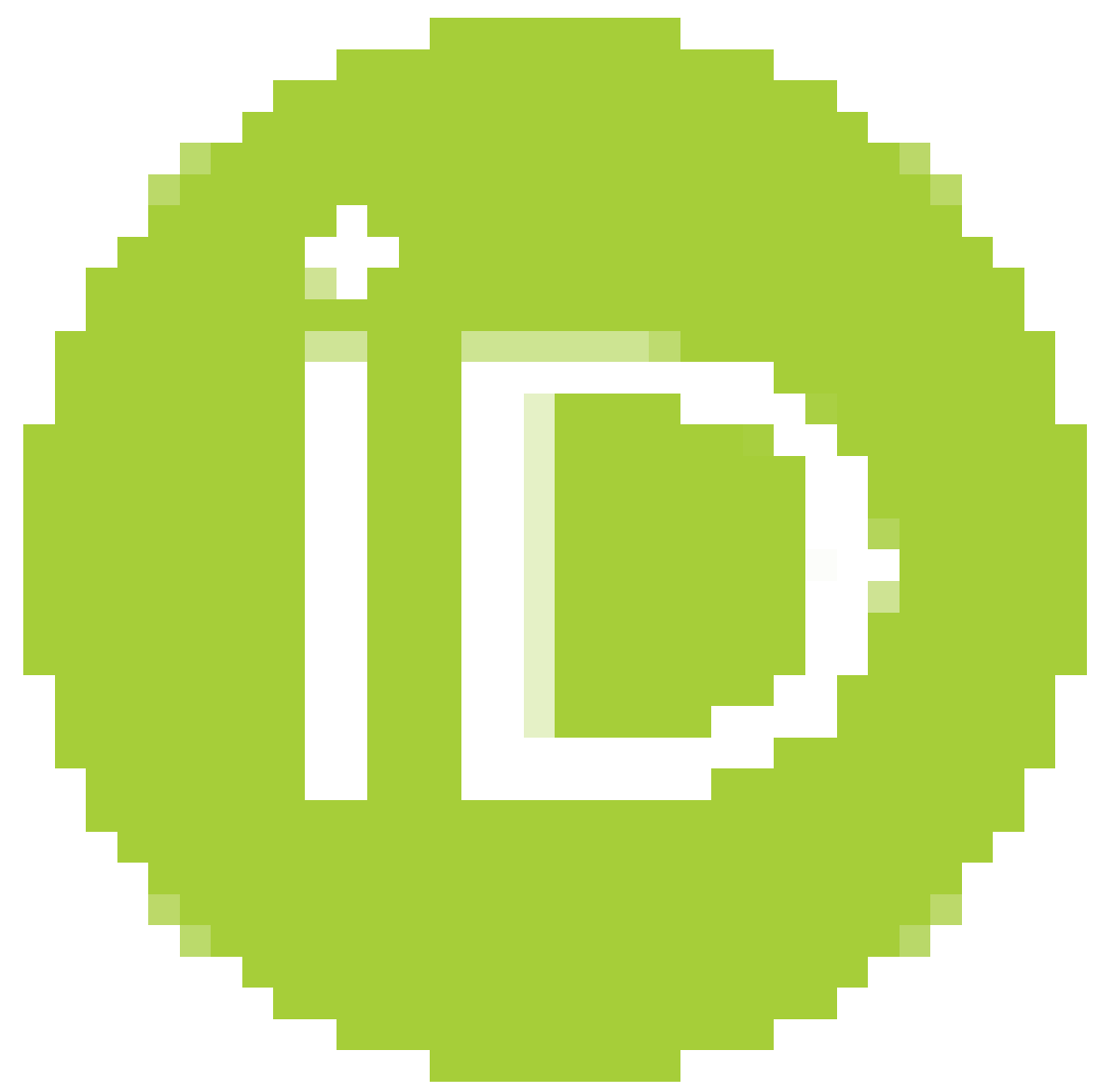

Figure 1. Mediators of cell death during cryopreservation and the effective apoptosis inhibitors during cryopreservation. 


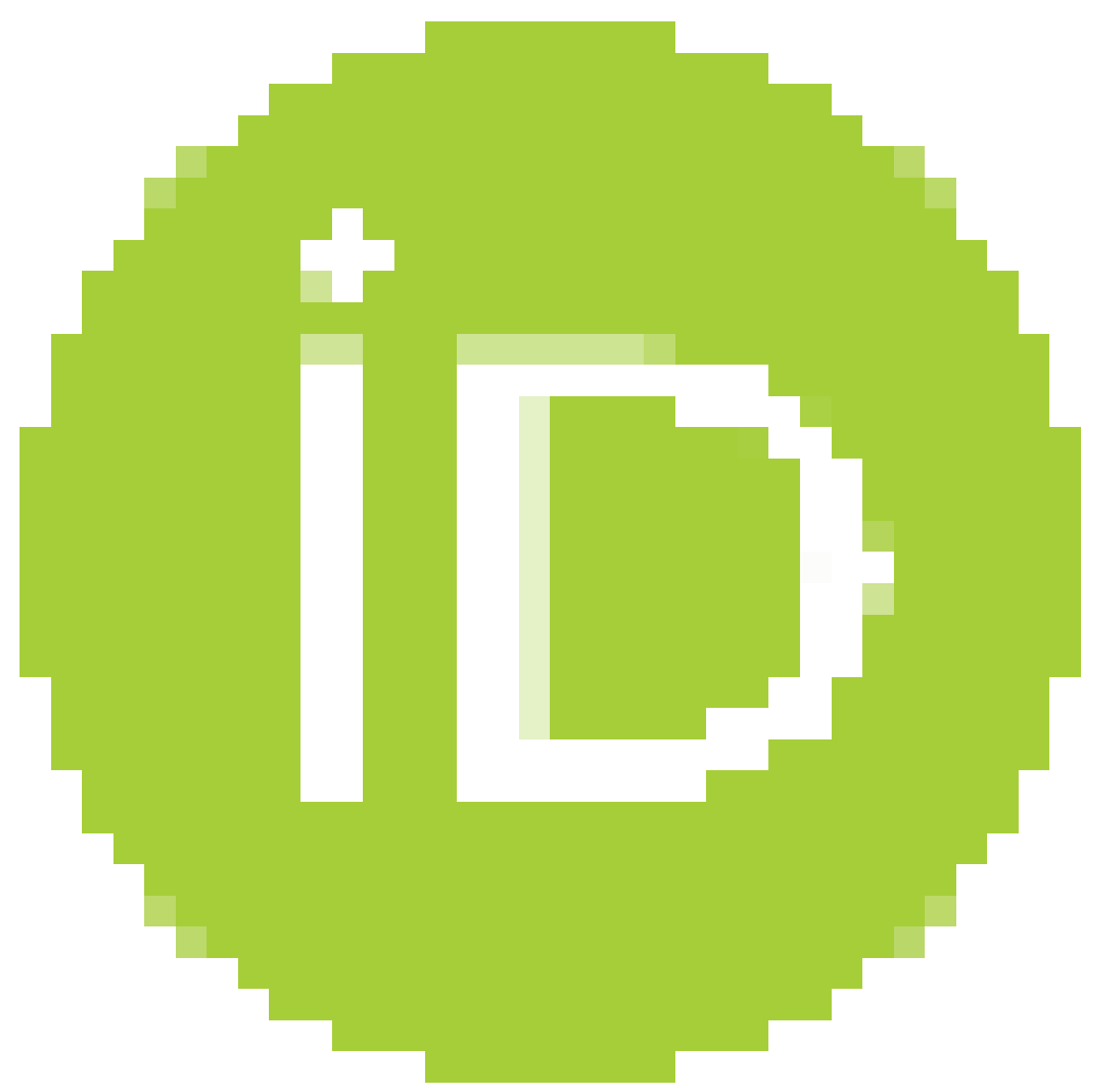

Figure 2. Schematic protocol for dry-preservation of Pv11 cells. Preincubation step: Pv11 cells were collected by centrifugation and resuspended into a $600 \mathrm{mM}$ preincubation trehalose mixture. The cell suspension was then incubated in a culture flask for $48 \mathrm{~h}$ at $25 \mathrm{C}$. Pictures show the aspect of Pv11 cells before and after preincubation. 


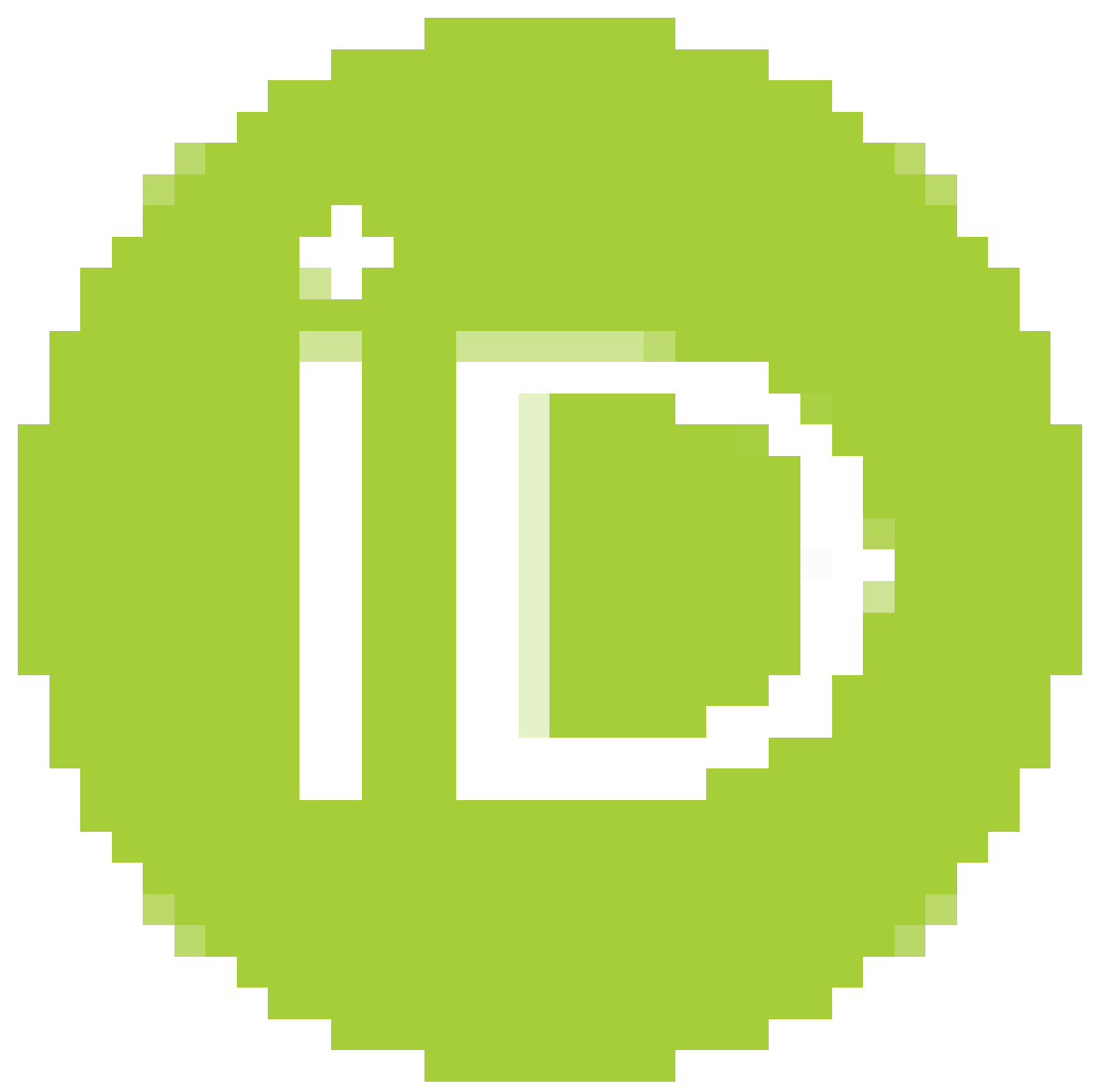

Figure 3. Schematic view of cryogel formation 


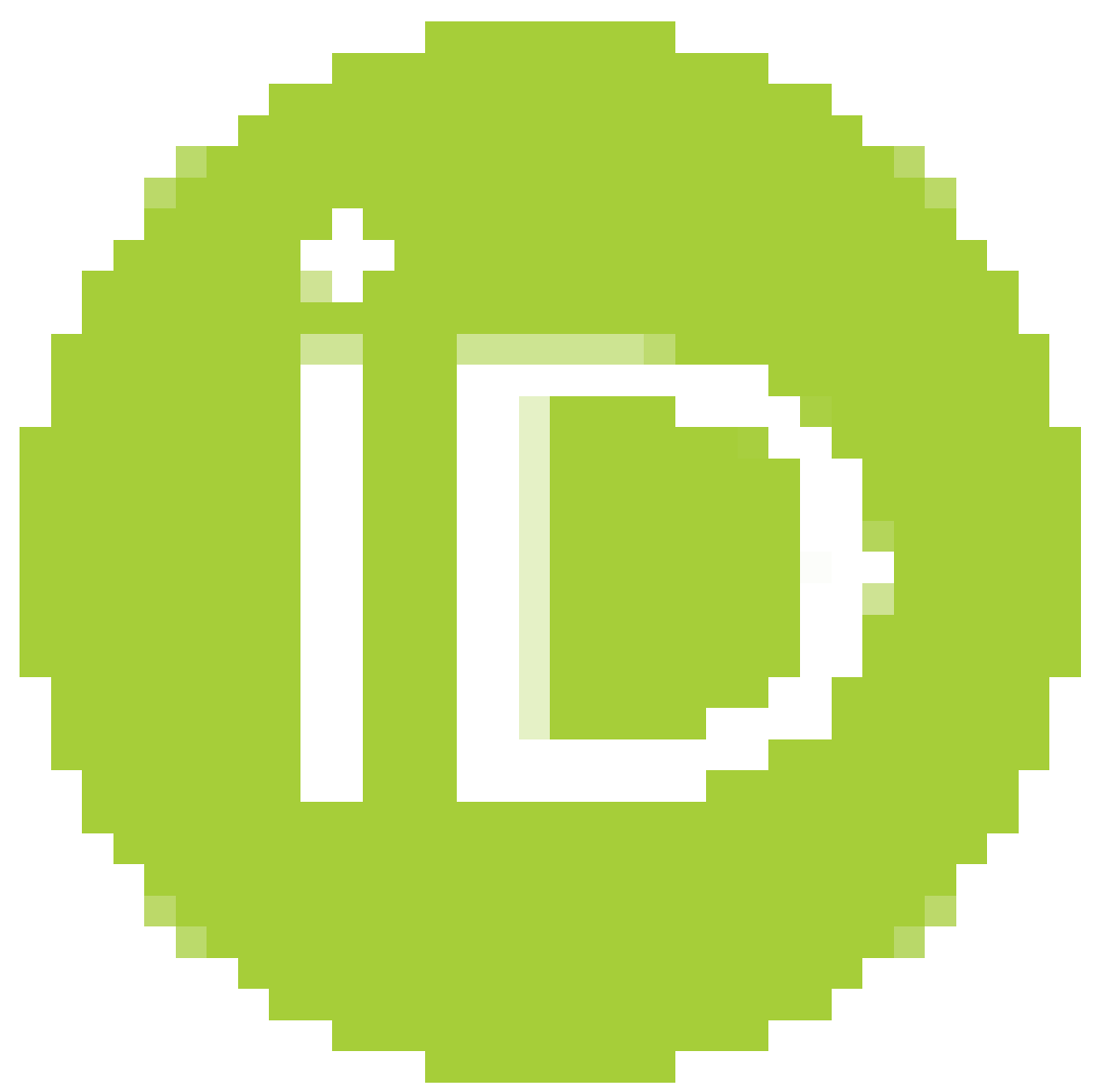

Figure 4. (A) For the formation of cryogels, (1) a hydrogel precursor solution is frozen $(\mathrm{T}<0 \mathrm{C})$. This process leads to a phase separation of the solvent (e.g., water) into a frozen phase (ice crystals) and a nonfrozen phase around ice crystals where the gel precursors (monomers, polymer, crosslinker, and initiator) are expelled. (2) Next, concentrated gel precursors are crosslinked around ice crystals (porogens). (3) Following cryogelation, thawed ice crystals give rise to a macroporous sponge-like hydrogel, known as a cryogel. (B) Digital image of (a) HA cryogels and (b) gelatin cryogels. (C) Scanning Electron Microscopy image of (a) HA cryogels and (b) gelatin cryogels. 


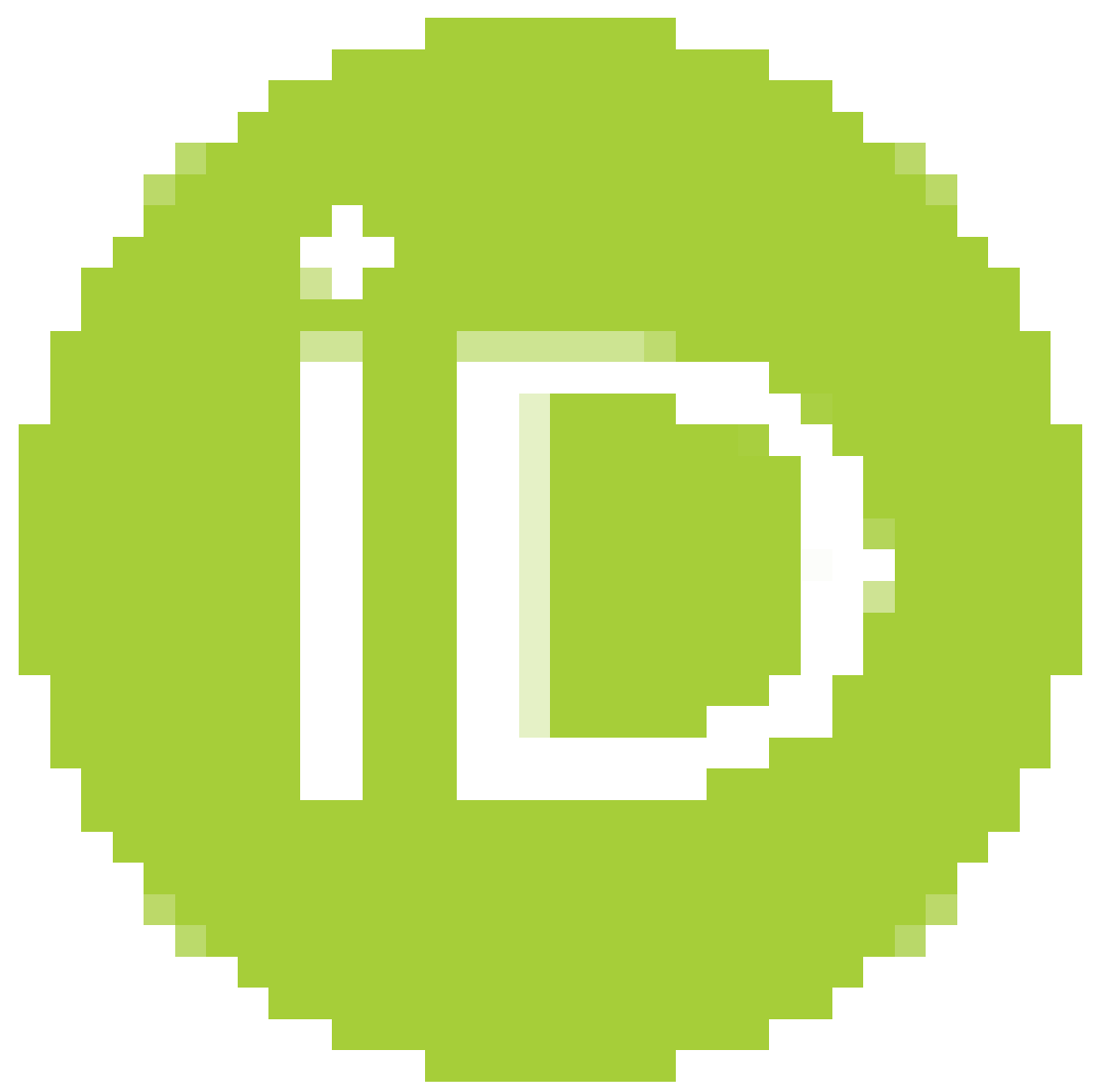

Figure 5. The setup of cells cultured on fibrin microbeads (FMB) in regular tubes placed in a slow rotator in a $\mathrm{CO} 2$ incubator (A). The cells grown in special tubes with a covered perforated cover for air exchange with preservation of sterility, as shown in (B). Attached cells on the surface of the FMB are shown in (B) by electron scanning microscopy $(\mathrm{C})$.

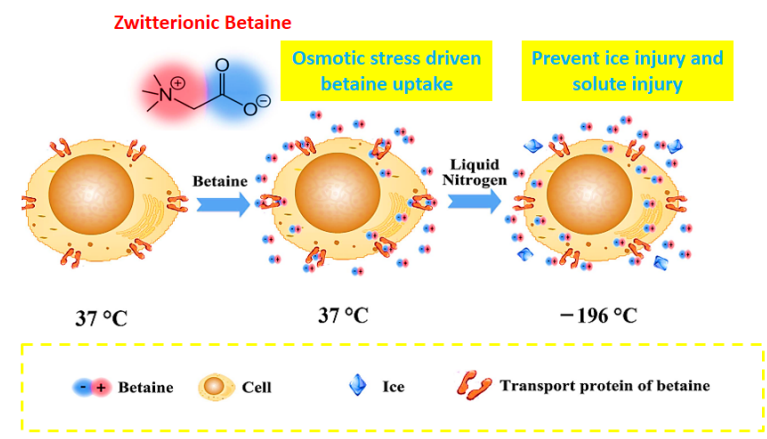

Figure 6. A proposed mechanism of cell cryopreservation using betaine with ultrarapid freezing. During the 
freezing process, the uptake of betaine by cells via transport proteins is induced by osmotic stress (middle) and prevents the intracellular and extracellular ice injuries as well as solute injury.
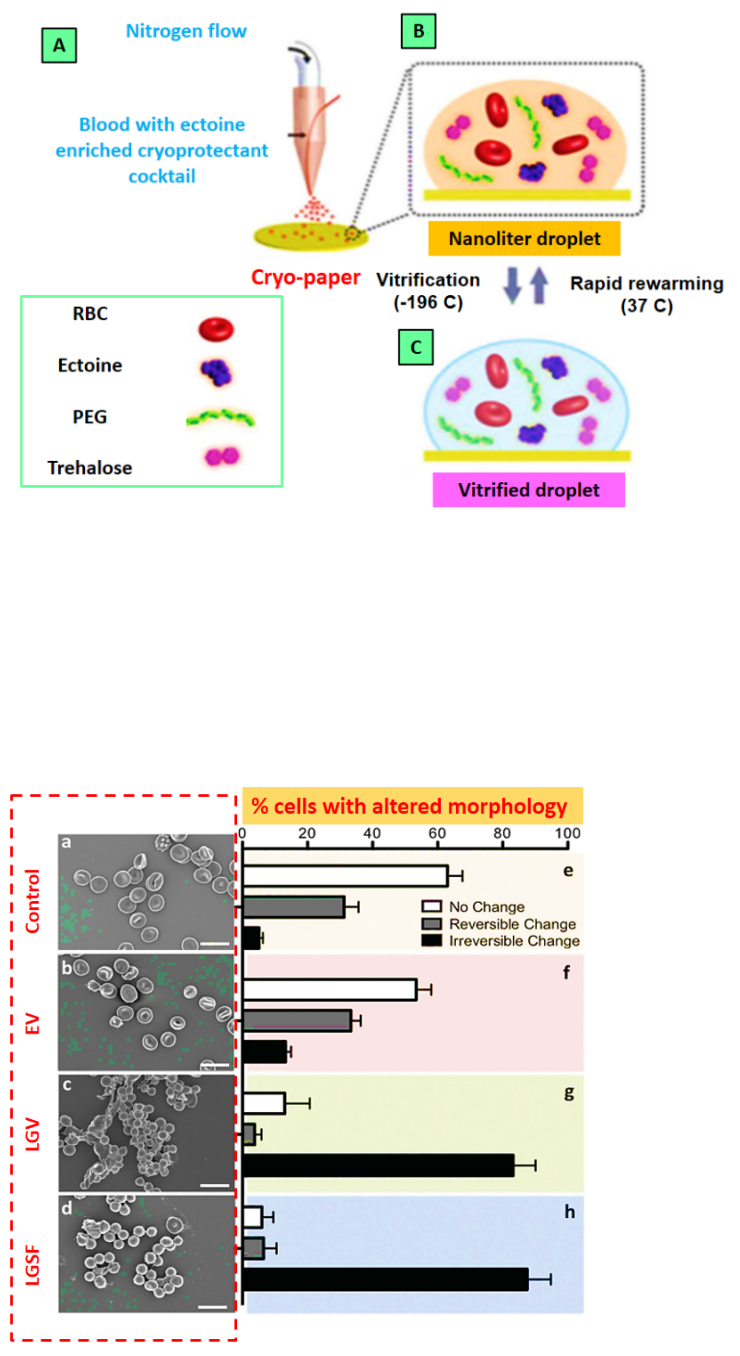


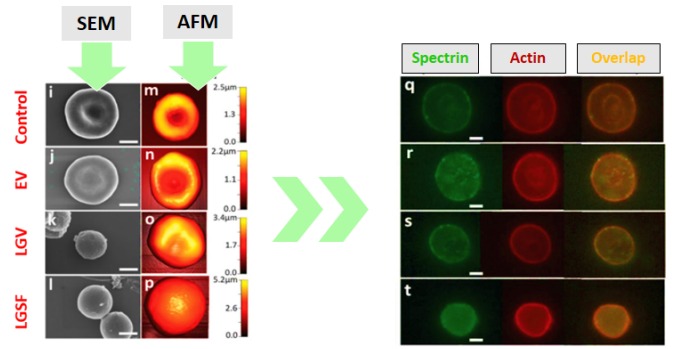

Figure 7. *Droplet-based vitrification of red blood cells. A) The essentials of the experimental setup for the droplet formation. B) Schematic magnified view of a single droplet on the cryo-paper including RBCs, ectoine, trehalose, and PEG. C) Schematic view of the vitrified droplet in (B). ${ }^{* *}$ Assessment of RBC morphology following ectoine-based vitrifi cation and rewarming. a-d) Scanning electron micrographs of RBCs: a) selected directly from blood, and recovered after: b) ectoine-based vitrifi cation (EV), c) lowglycerol vitrifi cation (LGV), and, d) low-glycerol slow freezing (LGSF). e-h) The percentage (\%) of RBCs within a population representing: e) untreated fresh blood, and blood recovered from: f) EV, g) LGV, and, h) LGSF: biconcave shape, and reversible and irreversible shape changes (n, number of cells $=114-309$, and $\mathrm{N}$, number of donors $=3-9$ ). Error bars in fi gures represent the standard error of the mean. $\mathrm{i}-\mathrm{l}$ ) Higher magnifi cation $(30000 \times)$ of SEMs of: i) fresh RBCs, and RBCs recovered after: j) EV, k) LGV, and, l) LGSF. $m-p$ ) Atomic force micrographs of: $m$ ) fresh RBCs, and RBCs recovered after: n) EV, o) LGV, and, p) LGSF. q-t) Fluorescence micrographs showing distribution of the spectrin-actin network in RBCs. q) RBCs in fresh blood, and RBCs recovered from: r) EV, s) LGV, and t) LGSF. Scale bar represents $10 \mu \mathrm{m}$ in a-d and $2 \mu \mathrm{m}$ in $\mathrm{i}-\mathrm{l}$ and $\mathrm{q}-\mathrm{t}$.

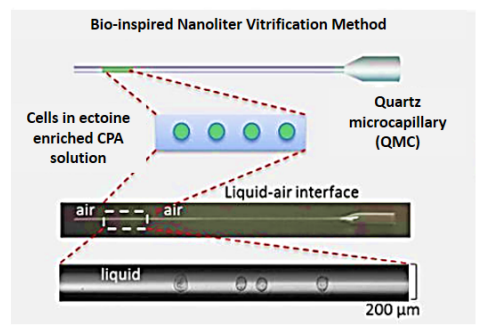

A) 


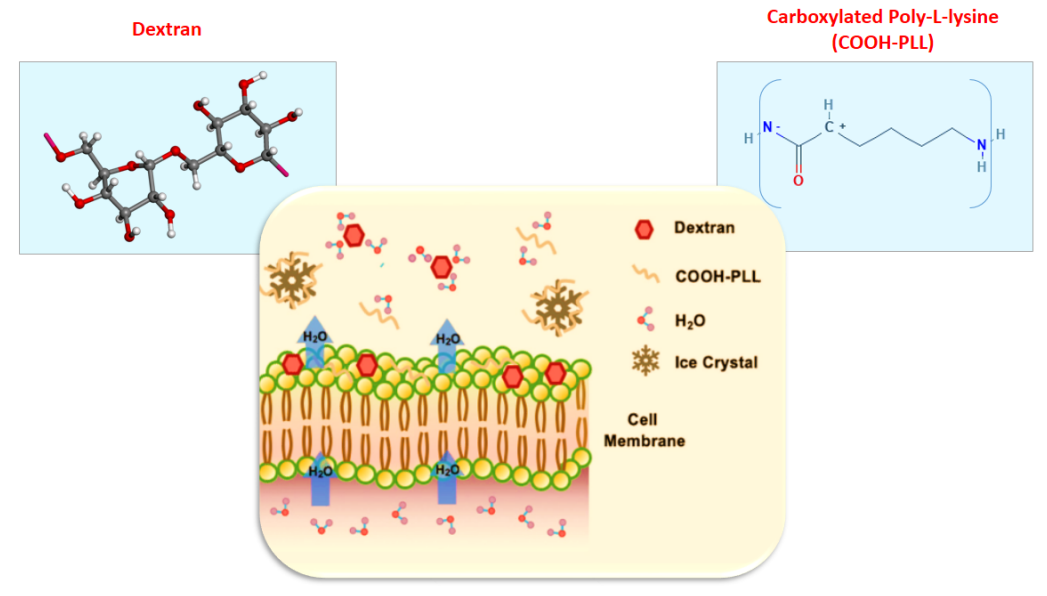

B)

C)

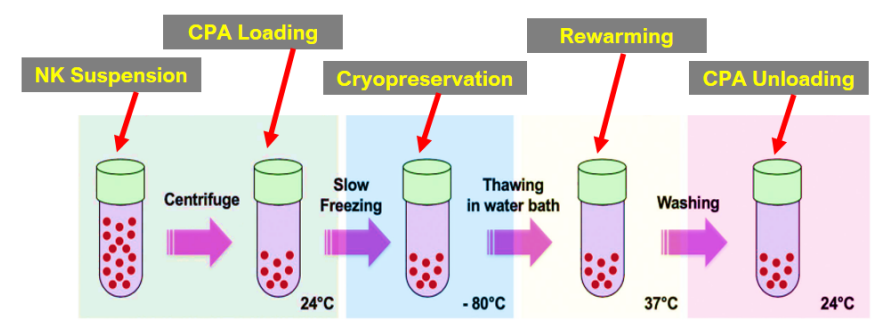

D)

Figure 8. A) Schematic view of bio-inspired nanoliter vitrification method. B) Schematic showing the chemical structures of dextran and carboxylated poly-L-lysine (CPLL). C) Schematic demonstrating the potential mechanism of action of dextran and CPLL during cryopreservation of natural killer (NK) cells. The synergic effect of CPAs is related to their high affinity to cell membrane, water molecules, and solutes. This characteristic might provide cell protection while removing intracellular water, restricting solute diffusion, and controlling the degree of dehydration to a level sufficient to minimize intracellular ice formation during cooling. Carboxylated PLL also might limit cryoinjury to cells by binding to ice crystals and inhibiting their growth and recrystallization during rewarming. D) Schematic showing the cryopreservation protocol used for preservation of natural killer (NK)-92 cells. 

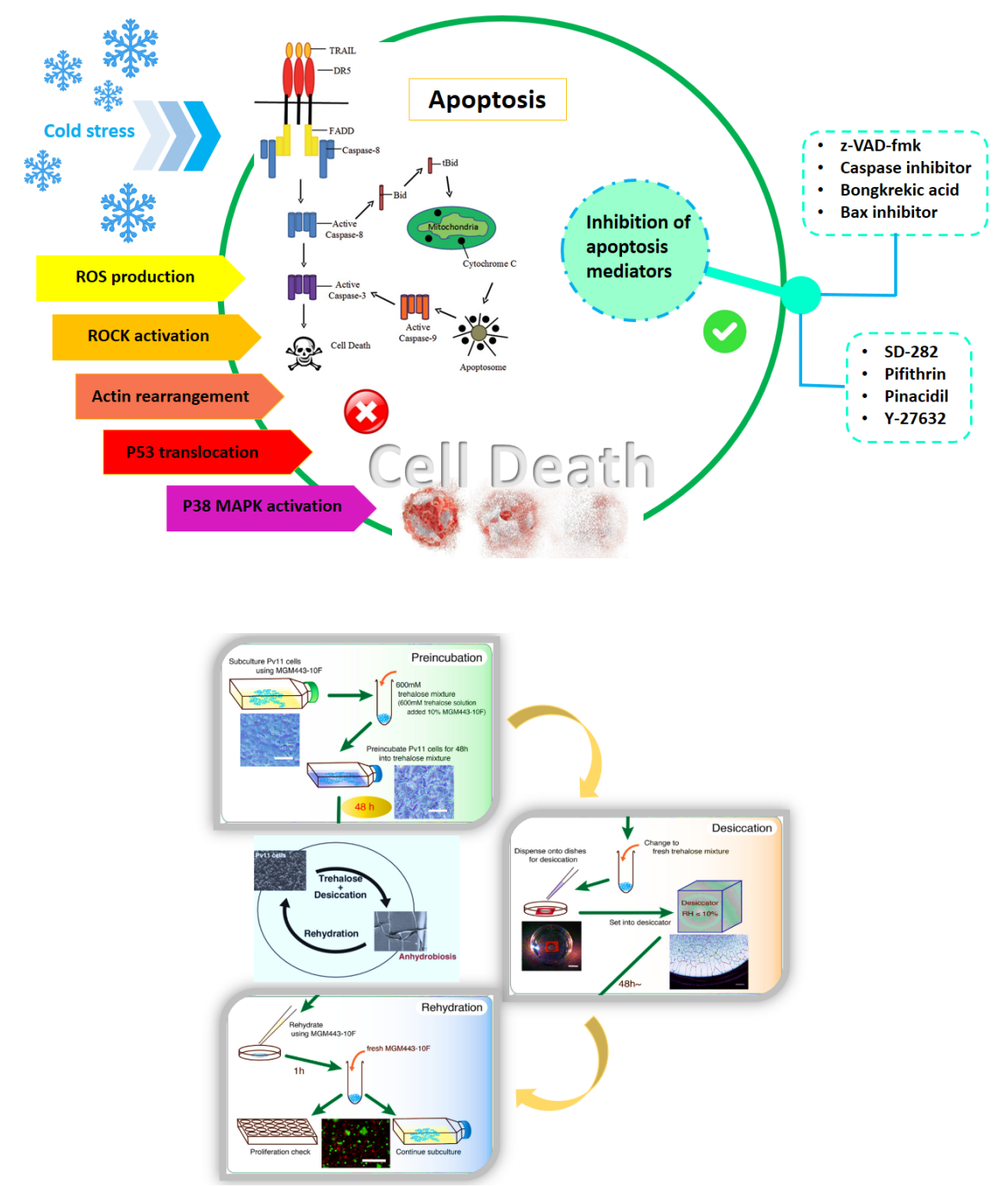

\begin{tabular}{|c|c|c|}
\hline & Slow freezing & Vitrification \\
\hline Characteristic & & \\
\hline Working time & More than $3 \mathrm{~h}$ & Fast, less than $10 \mathrm{~min}$ \\
\hline Cost & Expensive, freezing machine needed & Inexpensive, no special machine needed \\
\hline Sample volume $(\mu \mathrm{L})$ & $100-250$ & $1-2$ \\
\hline Concentration of CPA & Low & High \\
\hline Risk of freeze injury & High & Low \\
\hline Post-thaw viability & High & High \\
\hline Risk of toxicity of CPA & Low & High \\
\hline Status of system & Closed system only & Opened or closed system \\
\hline Potential contamination with pathogenic agents & Low & High \\
\hline Manipulation skill & Easy & Difficult \\
\hline
\end{tabular}



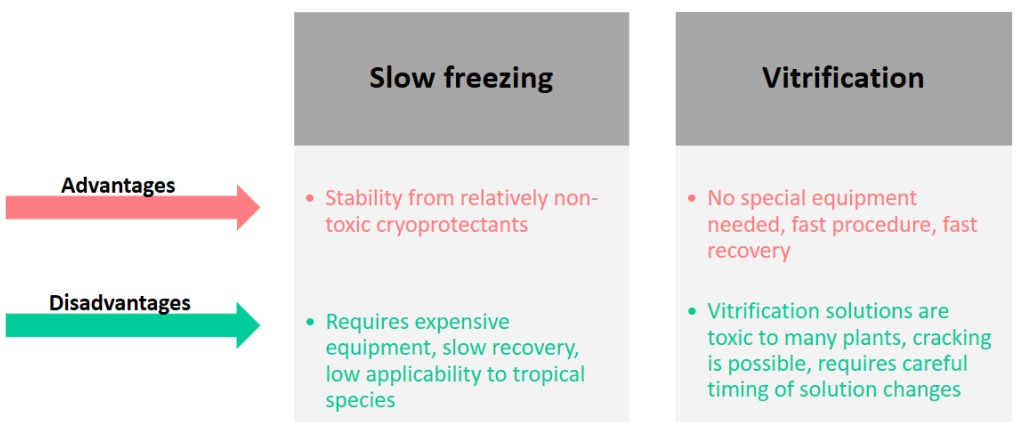

- No special equipment needed, fast procedure, fast recovery

- Vitrification solutions are toxic to many plants, cracking is possible, requires careful timing of solution changes
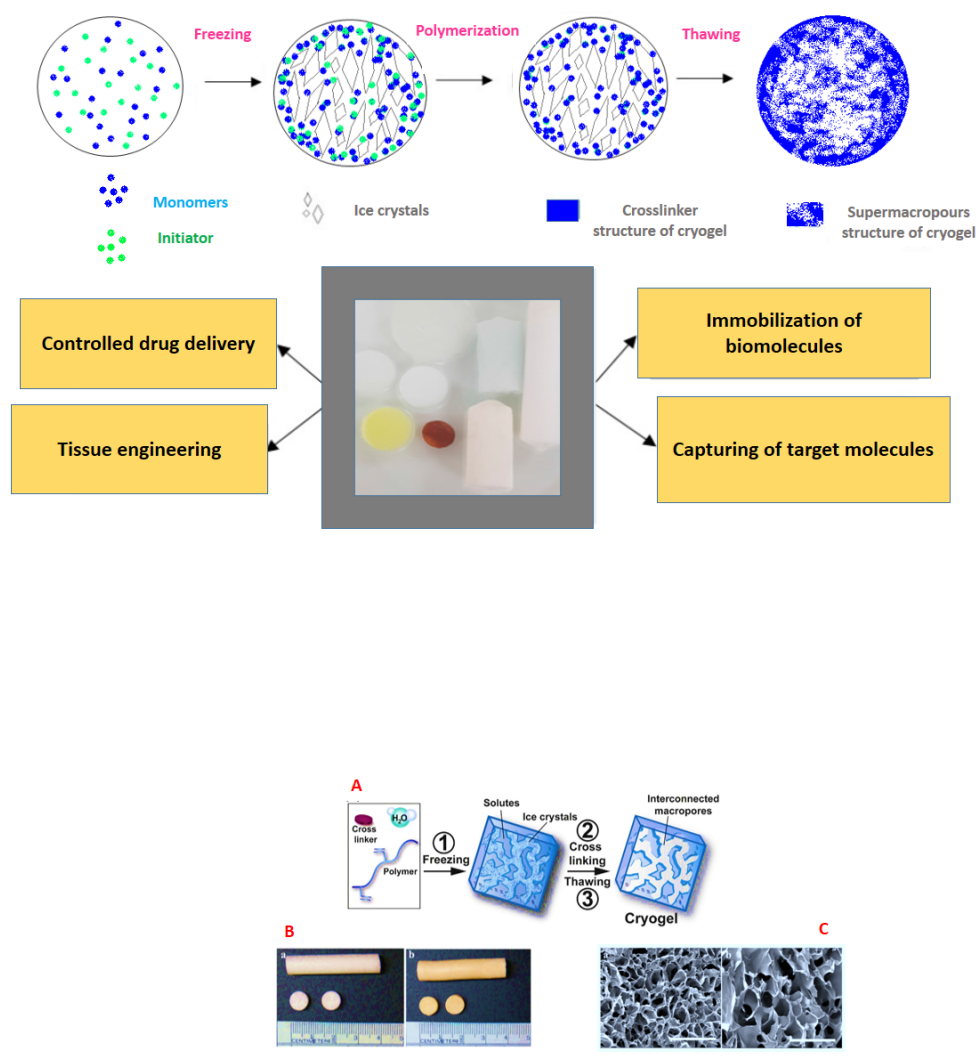

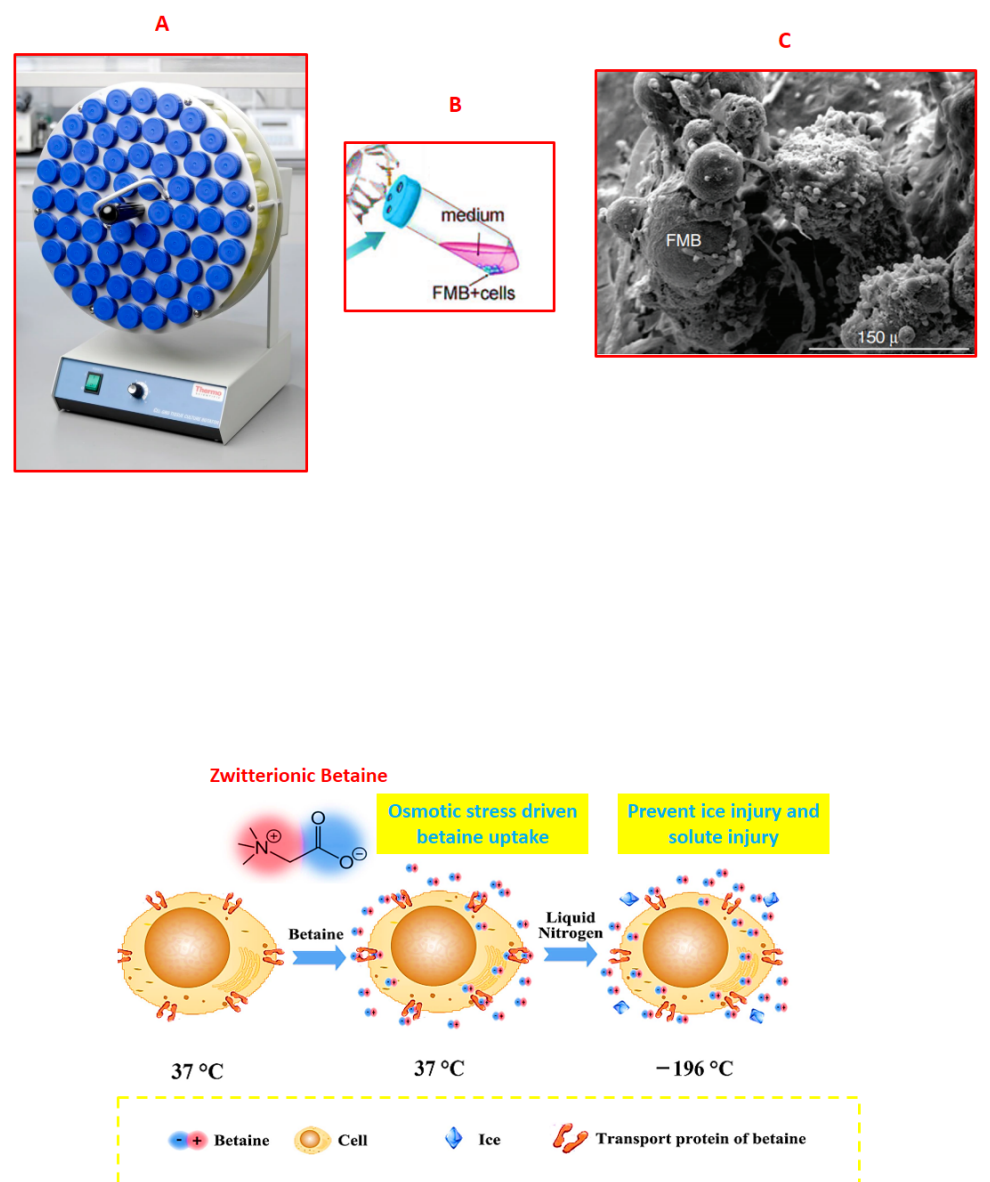

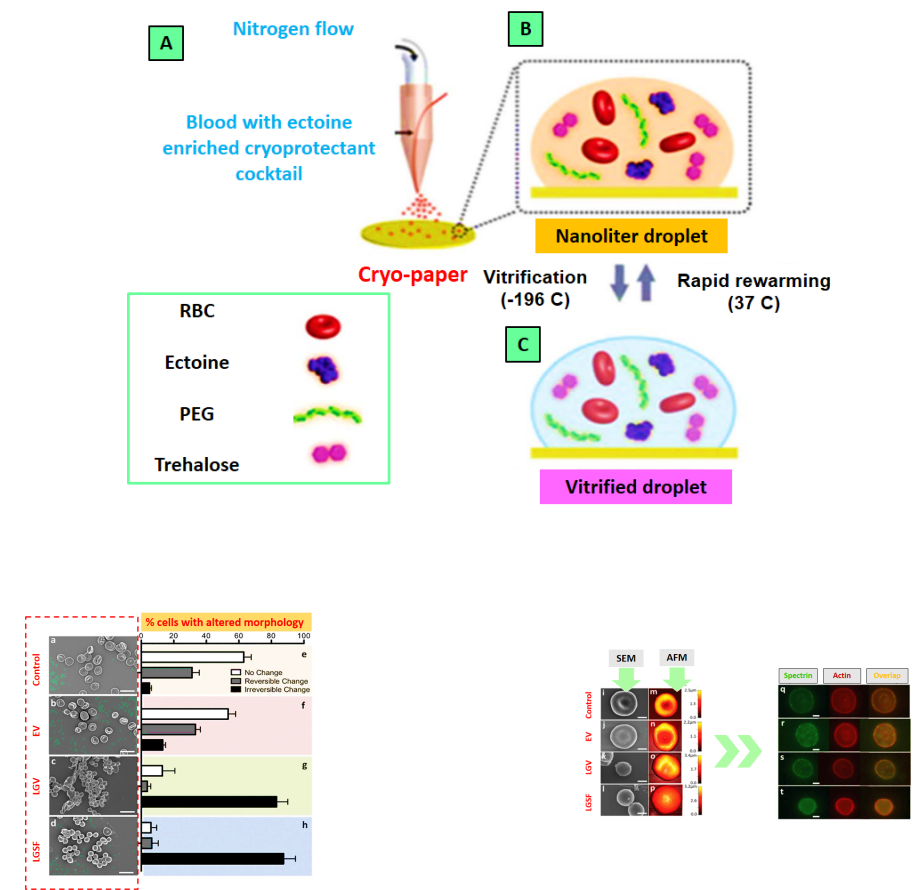

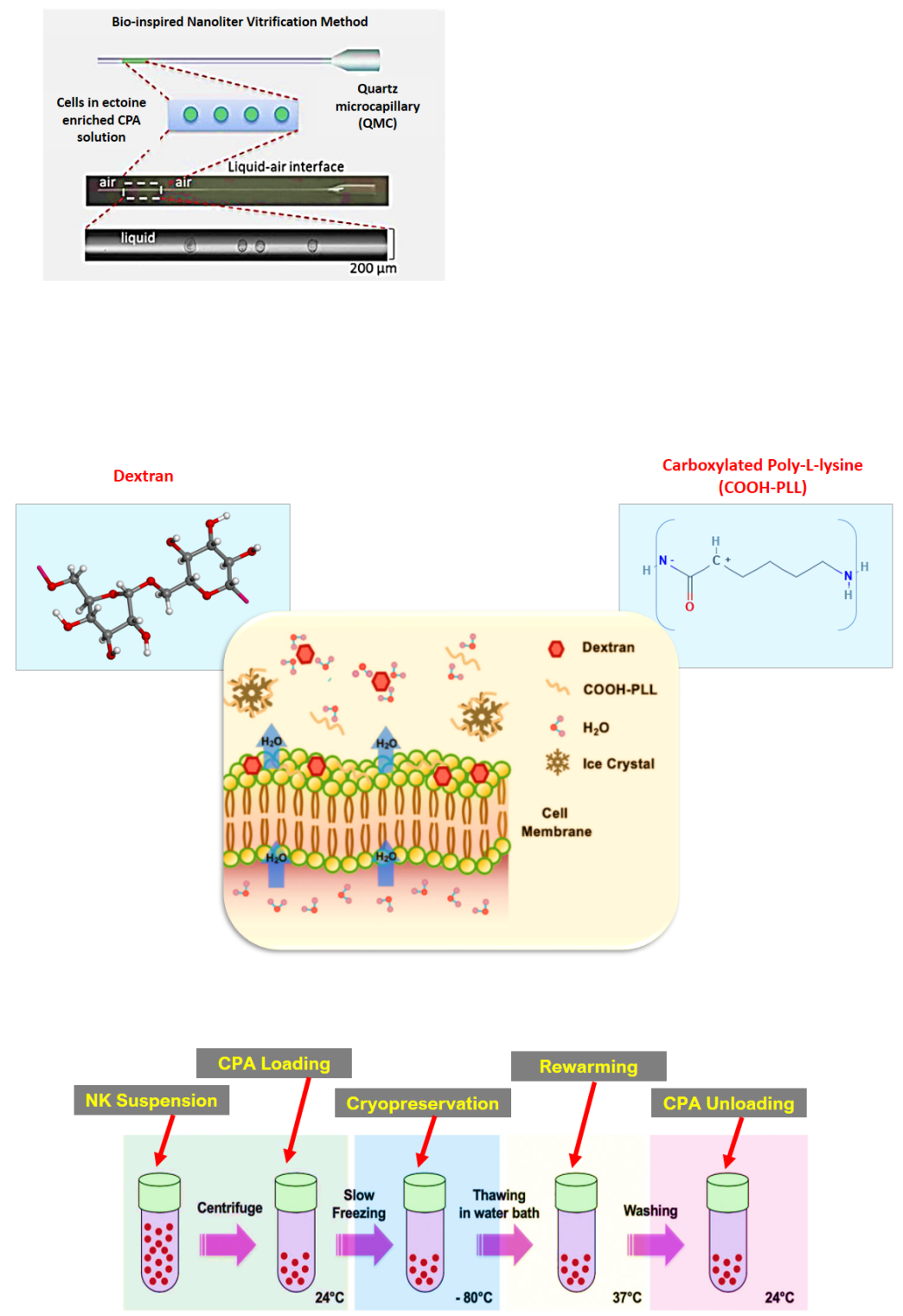


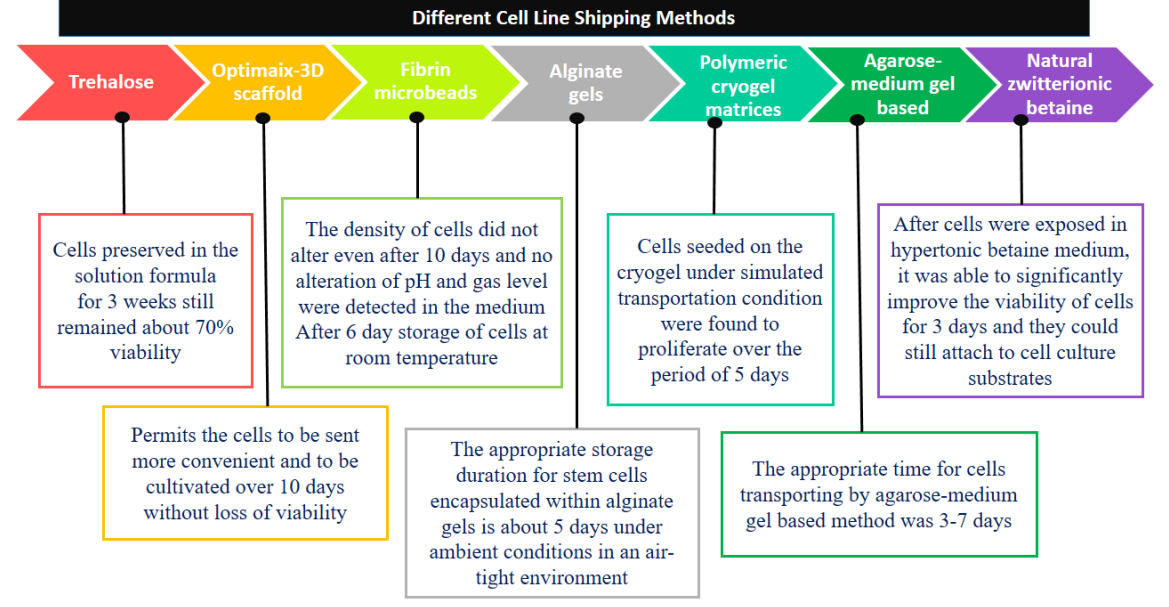




\title{
Recent developments in cell shipping methods
}

\author{
Shabnam Heydarzadeh ${ }^{1,2}$ \\ Mehdi Hedayati $^{2}$ (1)

\section{Sima Kheradmand $\mathrm{Kia}^{3}$ [(] | Seti Boroomand ${ }^{4}$ (ㅇ) |}

${ }^{1}$ Department of Biochemistry, School of Biological Sciences, Falavarjan Branch Islamic Azad University, Isfahan, Iran

${ }^{2}$ Cellular and Molecular Endocrine Research Center, Research Institute for Endocrine Sciences, Shahid Beheshti University of Medical Sciences, Tehran, Iran

${ }^{3}$ Laboratory for Red Blood Cell Diagnostics, Sanquin, Amsterdam, The Netherlands

${ }^{4}$ Djavad Mowafaghian Centre for Brain Health, Faculty of Medicine, University of British Columbia, Vancouver,

British Columbia, Canada

\section{Correspondence}

Mehdi Hedayati, Cellular and Molecular Endocrine Research Center, Research Institute for Endocrine Sciences, Shahid Beheshti University of Medical Sciences, Tehran 19395-4763, Iran.

Email: hedayati@endocrine.ac.ir and hedayati47@gmail.com

\begin{abstract}
As opposed to remarkable advances in the cell therapy industry, research reveal inexplicable difficulties associated with preserving and post-thawing cell death. Post cryopreservation apoptosis is a common occurrence that has attracted the attention of scientists to use apoptosis inhibitors. Transporting cells without compromising their survival and function is crucial for any experimental cell-based therapy. Preservation of cells allows the safe transportation of cells between distances and improves quality control testing in clinical and research applications. The vitality of transported cells is used to evaluate the efficacy of transportation strategies. For many decades, the conventional global methods of cell transfer were not only expensive but also challenging and had adverse effects. The first determination of some projects is optimizing cell survival after cryopreservation. The new generation of cryopreservation science wishes to find appropriate and alternative methods for cell transportation to ship viable cells at an ambient temperature without dry ice or in media-filled flasks. The diversity of cell therapies demands new cell shipping methodologies and cryoprotectants. In this review, we tried to summarize novel improved cryopreservation methods and alternatives to cryopreservation with safe and viable cell shipping at ambient temperature, including dry preservation, hypothermic preservation, gel-based methods, encapsulation methods, fibrin microbeads, and osmolyte solution compositions.
\end{abstract}

\section{KEYWORDS}

alternative methods, ambient temperature, cell culture, cell death, cell shipping, cell transport, preservation

\section{1 | INTRODUCTION}

One central aspect of cell science is the transportation of cells in particular conditions for long time intervals. Shipping cells across international borders can be problematic (Cobo et al., 2005; Nema \& Khare, 2012; Yao \& Asayama, 2017). There is a delay between the preparations for tissue regeneration and implantation in cell-based therapies. To sustain cells for this prolonged time, certain conditions, including appropriate $\mathrm{CO}_{2}$ concentration and temperature, must be established (Reddig \& Juliano, 2005). The development of alternative methods with minimal manipulation and maximal survival is preferable for clinical applications (Ehrenreich \& Ruszczak, 2006; Schoenhard \& Hatzopoulos, 2010). Despite the noteworthiness of this issue, it has scarcely focused on the prohibition of cell death in suboptimal conditions when cells are shipped to the clinic (Gorodetsky et al., 2011; Suuronen et al., 2008; Theus et al., 2008).

Abbreviations: CPA, cryoprotective agent; DMSO, dimethyl sulfoxide; ECM, extracellular matrix; EG, ethylene glycol; FMB, fibrin microbead; GLY, glycerol; GMP, good manufacturing practice; HA, HEMA agarose; hESC, human embryonic stem cell; HIF-1, hypoxia induced factor-1; hMSC, human mesenchymal stem cell; IVF, in vitro fertilization; LN2, liquid nitrogen; Me2SO, dimethyl sulfoxide; mESC, mouse embryonic stem cell; PG, propylene glycol; SEM, scanning electron microscope. 


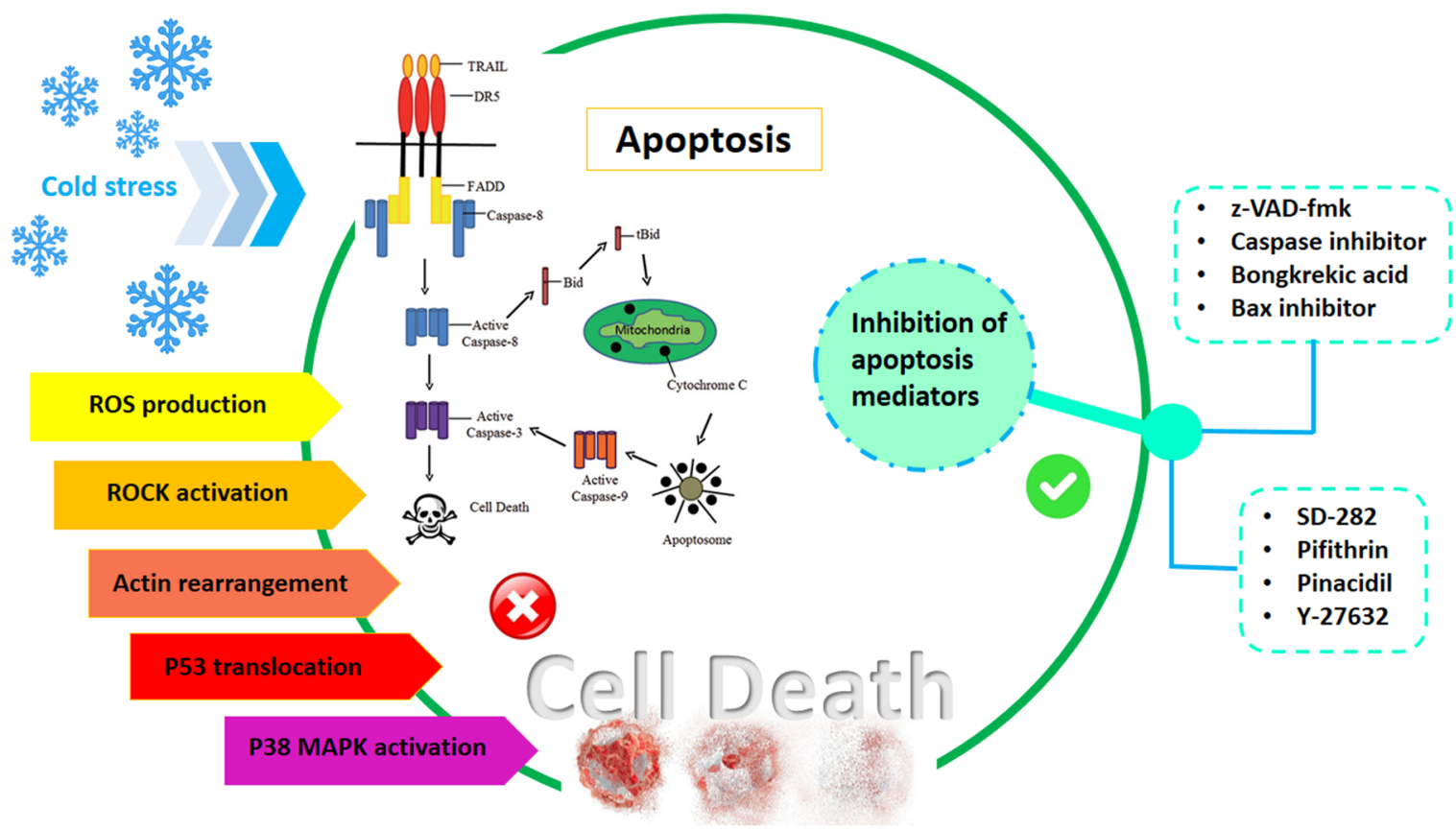

FIGURE 1 Mediators of cell death during cryopreservation and the effective apoptosis inhibitors during cryopreservation.

Figure 1 shows the mediators of cell death during cryopreservation and the effective apoptosis inhibitors (Bissoyi et al., 2014; Heng et al., 2006).

Cells are frequently transported from one city to another and from one country to another. There are some available strategies for cell shipping in cell culture-based research (Ebertz \& Locksley, 2004; Esfandiarei et al., 2007; Kumari \& Kumar, 2017). For many decades, the most well-known strategy for transferring cells between institutions and organizations was to ship cryopreserved cells on dry ice, usually treated with cryoprotective solutions such as dimethyl sulfoxide (DMSO), which is less toxic than many others ( $B$. Chen et al., 2013; Hanna \& Hubel, 2009). Cost of the process from storage to delivery is high. When cells are bathed in DMSO at room temperatures, it is cytotoxic. Additionally, in the case of solid carbon dioxide (dry ice) usage, an adequate mass is required to continue the shipping period with some contingency for shipping delays (Coopman, 2013; Grein et al., 2010; Stevens et al., 2007; Svalgaard et al., 2018). In some countries, the transportation of cells via this method is limited. According to Federal Express shipping information (obtained from http://www.fedex.com/us/dangerousgoods/index. html), more than $50 \%$ of countries worldwide forbid delivery with dry ice because of its hazardous nature, a possible reason for explosion, and suffocation (Wang et al., 2015). Because of their low cost, liquid nitrogen vapor shippers are ideal for transporting valuable cells and biological agents (Wang et al., 2015).

Cryopreservation is classified into slow freezing and vitrification methods (Table 1). Main dissimilarity between these two methods is the different dosages of cryoprotective agents (CPAs) and the cooling rates used (Gao \& Critser, 2000; Jang et al., 2017). The study of evaluation of the comparison between vitrification and controlled slow freezing methods under standardized conditions has been performed recently (Fan et al., 2009; Table 1). Because vitrification has more advantages than other cryopreservation methods, it still has some drawbacks and disadvantages. The advantages and disadvantages of slow freezing and vitrification techniques are summarized in this review (Table 2; Sharma, 2005). Cryopreservation is a procedure that preserves biological products by cooling them in low temperatures and slowing their vital cellular functions. Cellular events such as ice crystal formation, osmotic stress, and membrane destruction are the main reasons cells cannot be preserved with ordinary freezing methods at frequent intervals, and result in apoptosis (Jang et al., 2017). For these matters, optimizing post-cryopreservation cell survival and investigating the effect of various cooling or thawing rates for specific cell types are the focus of attention (Armitage, 1987; Meryman, 2007). Cryoprotectants are being studied through the optimization of cooling and thawing rates. The main properties of these chemicals for protection against cell damage within cryopreservation are the stabilization of the cell membrane (Rubinstein et al., 1995; Rudolph \& Crowe, 1985) and, or the decreasing of osmotic effects (Baust et al., 2003). The optimal cooling rates are related to cell types because there are different cell potencies to shift water across the membrane. Different cell types show specific biological features (Stacey \& Masters, 2008). Environmental changes may cause cell damage during the freezing process (Miyamoto et al., 2018). During the cryopreservation, slow freezing rates can cause cell injury and harm cell viability. At the same time, high concentrations of cryoprotectant can cause cell toxicity. To avoid toxins, the ideal cooling rate and CPA should be used (Heo et al., 2015).

Ambient temperature shipping is a modern method of transferring cells. Although cells can be stored at $-78.5^{\circ} \mathrm{C}$ for an extended 
TABLE 1 Comparison between the slow-freezing and vitrification methods

\begin{tabular}{|c|c|c|}
\hline Characteristic & Slow freezing & Vitrification \\
\hline Work time & More than $3 \mathrm{~h}$ & Fast, less than $10 \mathrm{~min}$ \\
\hline Cost & $\begin{array}{l}\text { Expensive, freezing } \\
\text { machine needed }\end{array}$ & $\begin{array}{l}\text { Inexpensive, no special } \\
\text { machine needed }\end{array}$ \\
\hline Sample volume $(\mu \mathrm{l})$ & $100-250$ & $1-2$ \\
\hline Concentration of CPA & Low & High \\
\hline Risk of freeze injury & High & Low \\
\hline Post-thaw viability & High & High \\
\hline Risk of toxicity of CPA & Low & High \\
\hline status of system & Closed system only & Opened or closed system \\
\hline $\begin{array}{l}\text { Potential contamination with } \\
\text { pathogenic agents }\end{array}$ & Low & High \\
\hline Manipulation skill & Easy & Difficult \\
\hline
\end{tabular}

Abbreviation: CPA, cryoprotective agent.

TABLE 2 Advantages and disadvantages of commonly used cryopreservation techniques

\begin{tabular}{|l|ll|}
\cline { 2 - 3 } \multicolumn{1}{c|}{ Advantages } & Slow freezing & Vitrification \\
\cline { 2 - 3 } & $\begin{array}{l}\text { Stability from relatively non-toxic } \\
\text { cryoprotectants }\end{array}$ & $\begin{array}{l}\text { No special equipment needed, fast procedure, fast } \\
\text { recovery }\end{array}$ \\
\cline { 2 - 3 } Disadvantages & $\begin{array}{ll}\text { Requires expensive equipment, slow recovery, } \\
\text { low applicability to tropical species }\end{array}$ & $\begin{array}{l}\text { Vitrification solutions are toxic to many plants, cracking } \\
\text { is possible, requires careful timing of solution changes }\end{array}$ \\
\hline
\end{tabular}

period of time, DMSO toxicity will destroy the cells due to decreasing viability, depletion of dry ice, and thawing of the sample during shipment (Vigneault et al., 2009). DMSO has been described as toxic to stem cells at room temperature. The ability to preserve cells is profound for their application in therapeutical and research studies. With the various types of cells required to preserved, attentive optimization of preservation strategies seems tiresome and shows notable restriction (B. Chen et al., 2013; Heo et al., 2015; Li, 2007; Michael et al., 2010). For preservation of the great diversity of cells, accurate optimization of preservation protocols seems tiresome and indicates remarkable limitations. So far, no survey papers have published summarizing different cell shipping methods at ambient temperature. All known reviews are concerned either with particular cases of DMSO alternatives. In this review, we will focus on summarizing the findings of recent novel preservation studies that have been performed.

\section{2 | SEARCH STRATEGY}

A systematic literature search has been performed using PubMed, Web of Science, Google Scholar, Scopus, and Science Direct as an international database from 1978 to 2020 with English language restrictions. The search keywords were: Cell shipping, cell transport, cell culture, and preservation. All English language and full-text papers were selected.

\section{3 | ALTERNATIVES TO DMSO FOR CRYOPRESERVATION}

\section{1 | Glycerol, proline, and ectoine}

The commonly used CPA is DMSO. DMSO is recognized to show toxic effects, reduce the expression of critical factors related to stem, and induce epigenetic changes. Hence, there is a formal request from health care authorities to discover nontoxic cryopreservation alternatives to DMSO (Svalgaard et al., 2018). The duties of DMSO as a supplement in the cryopreservation medium are to guard the cells against excessive dehydration until the freezing time and to prevent intracellular ice formation. However, while it gives a high rate of cell survival during the frozen period, it also causes chemical cytotoxicity at ambient temperature, which can cause osmotically damaging cell injury. Consequently, fewer cytotoxic CPAs are required to standardize this course of action (Janz et al., 2012; von Bomhard et al., 2016; Syme et al., 2004).

Since $\mathrm{Me}_{2} \mathrm{SO}$ is toxic and has notable side effects, besides the pathogen transferring feature of serum, both are unacceptable for 
hMSC storage in clinical applications. Additionally, $\mathrm{Me}_{2} \mathrm{SO}$ can cause uncontrolled differentiation of stem cells. Nontoxic alternatives to $\mathrm{Me}_{2} \mathrm{SO}$ include Glycerol, Proline, and Ectoine (Hegner et al., 2005; Ji et al., 2004; Woods et al., 2007). Glycerol prevents osmotic damage by reducing intracellular water loss and intra and extracellular ice formation (Morris et al., 2006). Glycerol was successful in cryopreservation of blood cells and adult stem cells (De Vries et al., 2004; Scott et al., 2005). Ectoine was detected in Ectothiorhodospira halocholris to protect against high salt concentrations and temperatures. Ectoine has already been used to stabilize cells during the freezing time. This function originated from the stabilization of proteins and enzymes and the regulation of the cell's osmotic balance. Proline is a unique cryoprotectant, as it has low- and macromolecular features. Proline can diffuse into the cells and forms hydrogen bonds typical for low-molecular cryo-protection. Depending on the concentration, Proline forms aggregates that act like macromolecular cryoprotectants (Grein et al., 2010; Troitzsch et al., 2008). Proline can preserve enzyme activity and membrane stability (Rudolph \& Crowe, 1985) as well as increase the post-thaw vitality of spermatozoa (Li et al., 2003). The use of pentaisomaltose as an extracellular cryoprotectant has been agreed for clinical utilization. It is less likely than DMSO to interact with the intracellular molecules. The comparison of cryopreservation in DMSO or Pentaisomaltose has been evaluated in vitro cell survival as well as long-term cryopreservation in vivo models (Svalgaard et al., 2018).

\subsection{Zwitterionic betaine molecule and polymers}

Betaine is a good CPA, zwitterionic, and hydrophilic molecule found in microorganisms, plants, and animals. Betaine has an efficient action on ice formation and can regulate osmotic stress. Betaine is a wellknown osmoprotectant capable of protecting cells against osmotic stress (Courtenay et al., 2000). Also, zwitterionic Betaine produces water protection around the proteins to control their stability and functions. Moreover, Betaine is related to the freezing tolerance of various plants. According to Kishitani et al. studies, the leaves of winter-type barley accumulate Betaine at high levels during cold conditions to protect against freezing damage (Nomura et al., 1995). J. Yang et al. (2016) represented Betaine-based cell cryopreservation as a nontoxic alternative to DMSO. According to their findings, ultrarapid freezing was more acceptable than the conventional controlledrate freezing method due to diverse cell types' cryopreservation, better post-thaw survival rate, and low toxicity.

The interaction of water molecules had shown an exact relation with ice crystallization. For this reason, for minimizing ice formation, CPAs are commonly hydrophilic molecules indicating the most potent inhibition to water crystallization and excellent capability of decreasing the water chemical potential. Zwitterionic betaine has a highly hydrophilic feature and can bind water molecules through its ionic solvation function and charge groups (Shao \& Jiang, 2015). As well as ice damage, the solute injury that occurs by osmotic stress is also the cause of cell death at the time of the freezing and thawing process. Following cells exposed to hypertonic betaine medium, they could still assume their spindle shape was similar to the control cells. Excitingly, the presence of betaine in the hypertonic $\mathrm{NaCl}$ medium can improve cell viability. These events indicate that by the accumulation of betaine, cells will make adjustments to external osmotic stress. Therefore, betaine shows the power to protect cells from solute injury. Betaine is a natural zwitterionic molecule and nontoxic CPA that enables cells to survive ultra-rapid cryopreservation. The ultra-rapid cellular uptake of Betaine is obvious for intracellular protection during the freezing period. Due to betaine's privileged cell cryopreservation ability, it can be an alternative CPA to the conventional toxic ones (J. Yang et al., 2016). Figure 2 proposes cell cryopreservation based on Betaine with ultrarapid freezing.

M. Liu et al. were the first to report a zwitterionic compoundbased method for chondrocyte cryopreservation. They combined a molecule of zwitterionic with its polymers without the need for DMSO. To achieve a higher cryo-protection efficacy, poly (sulfobetaine methacrylate) and poly (carboxybetaine methacrylate) were selected (extracellular CPA) and combined with zwitterionic betaine (intracellular CPA). The post-thaw survival efficiency for the samples

Zwitterionic Betaine
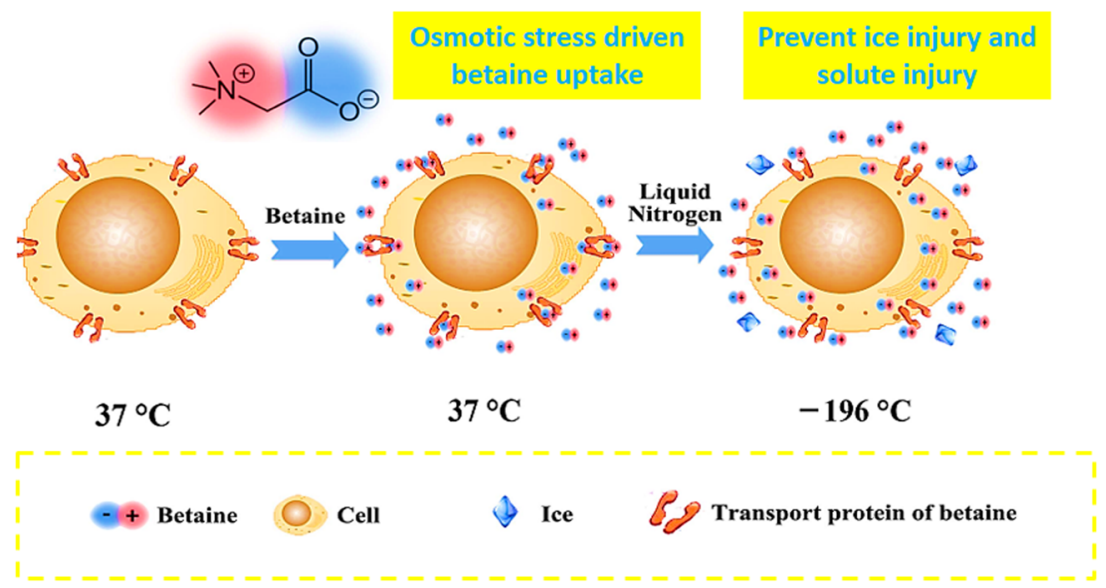

FIGURE 2 A proposed mechanism of cell cryopreservation using betaine with ultrarapid freezing. During the freezing process, the uptake of betaine by cells via transport proteins is induced by osmotic stress (middle) and prevents intracellular and extracellular ice injuries as well as solute injury. 
treated with Betaine alone was $57 \%$ at the concentration of $4 \%$ betaine. The combination of zwitterionic betaine with poly (sulfobetaine methacrylate) and poly (carboxybetaine methacrylate) increased chondrocyte optimum efficiency to $85 \%$ and $91 \%$, respectively. Impressively, this efficiency was already higher than that of the traditional freezing method. Therefore, they added extracellular zwitterionic that shows the biocompatibility and hydration ability for betaine uptake. Indeed, it has been reported that zwitterionic polymers could not enter cells for intracellular cryo-protection. When zwitterionic polymer has been used alone, it represented the lower cell survival rates, similar to those of the control samples (Liu et al., 2019).

\subsection{Alginate polymer and zwitterionic betaine}

It has been reported that in cell therapy and tissue engineering studies, DMSO can cause some side effects such as inhibition of the enzyme, abnormalities in cell metabolism, apoptosis, and uncontrolled differentiation of stem cells. Thus, immediate complex washing procedures, which can result in inevitable cell damage, are needed for DMSO removal. Alginate polymers can contribute to extracellular protections and exhibit a mild gelation process with the capability for post-thaw cell usage. Alginate polymers, with their hydrophilic property, can bind to the water molecules and decrease the freezing water degree for cell cryo-protection. Therefore, these polymers are novel CPAs for direct encapsulation of postcryopreserved cells during tissue engineering. Zwitterionic betaine is known as a nontoxic CPA with great potential of being an alternative for DMSO. It can enter cells to keep them safe from ice and osmotic injury during the cryopreservation process. According to Yang J. et al., studies, the earliest alginate polymer-based method was reported for human chondrocyte cryopreservation. This polymer combined with zwitterionic betaine as a natural osmoprotectant for intracellular protection. In this way, after adding cells to the CPA solutions and incubating at $37^{\circ} \mathrm{C}$, cells will be transferred into a liquid nitrogen tank. The cryopreservation efficiency reached $~ 90 \%$ and showed regular cell functions for 14 days (J. Yang et al., 2019).

\section{4 | Bio-inspired cryo-ink}

A critical concern in conventional vitrification is the ice crystallization within rapid rewarming. It comes from the latent heat of fusion that leads to cell hemolysis. Recently, a new technology-enabled capability of printing viable and functional cells is reported. To avoid such crystallization, a novel and innovative cryo-ink was unified with a cryo-printer to convert a massive volume of human blood into Nano liter droplets on cryo-paper. In this method, the CPA solution included Ectoine, Trehalose, and Polyethylene glycol. The cryo-ink as a CPA can assist the cells in overcoming the stress at the time of the cooling and rewarming processes. The cryo-ink solution used in this study posed complementary enhancing effects. Ectoine is present in extremophilic bacteria, which can adapt to high thermal and osmotic shock conditions. Indeed PEG affects the freezing rate of the solution. Crystallization can be avoided by ultra-rapid rewarming through adding the printed nano-liter droplets to cryo-paper in media $\left(\geq 37^{\circ} \mathrm{C}\right)$. Furthermore, utilizing bio-inspired CPAs such as Ectoine and Trehalose eliminates extra washing times (Figure 3). Analyzing RBC morphology after Ectoine-based vitrification (EV) and rewarming indicated that approximately $88 \%$ of RBCs maintained their original form with low irreversible morphological alterations compared to low-glycerol slow freezing and low-glycerol vitrification methods. Furthermore, they measured band-3 phosphorylation, CD35 expression, intracellular nitric oxide, and reactive oxygen species for evaluating the function of EV recovered RBCs. In a vitrification process with Nano liter droplets, low concentrations of CPA (4.5\%) are needed, while in the traditional methods, high concentrations of CPAs (20\%-40\%) are required. High concentrations of CPAs loaded into large samples of RBC during conventional methods were revealed to have adverse effects (El Assal et al., 2014).

Current cryopreservation protocols based on DMSO entail deterioration in NK cell viability associated with side effects in humans. For this reason, in another recent research performed by El Assal et al. (2019), the cryopreservation of human natural killer cells was done by a slow freezing method. A cocktail of biocompatible bio-inspired cryo-protectants, including dextran and carboxylated -poly-L-lysine, was used in this method. They assessed the morphology of RBC following Ectoine-based vitrification and rewarming (Figure 4). It is essential to note that cryopreservation via this method showed higher cytotoxic potency against leukemia cells compared to cryopreservation with DMSObased solutions (El Assal et al., 2019). Dextran acts as a cryoprotective for various cell types. Carboxylated PLL, similar to antifreeze proteins (AFPs), inhibits ice crystal formation and recrystallization in cryopreservation. They demonstrated a synergic cryoprotective effect by mixing dextran and carboxylated PLL. These molecules bind strongly to water and thus can remove intracellular water during the freezing process. The mixture of dextran and CPLL can interconnect with concentrated salts to provide a sufficient rate of dehydration and avoid intracellular ice formation in the freezing process (Jain et al., 2014).

\section{4 | ALTERNATIVES TO CRYOPRESERVATION}

The contents reviewed in this study illustrate ongoing interest in introducing novel preservation methods for cell shipping. The time of storage for transportation is the determining factor in the selection of various cell shipment techniques. In this paper, we propose a variety of strategies for transportation processes with varying time requirements. Figure 5 depicts the various storage durations attained with the various preservation techniques discussed in this review. 

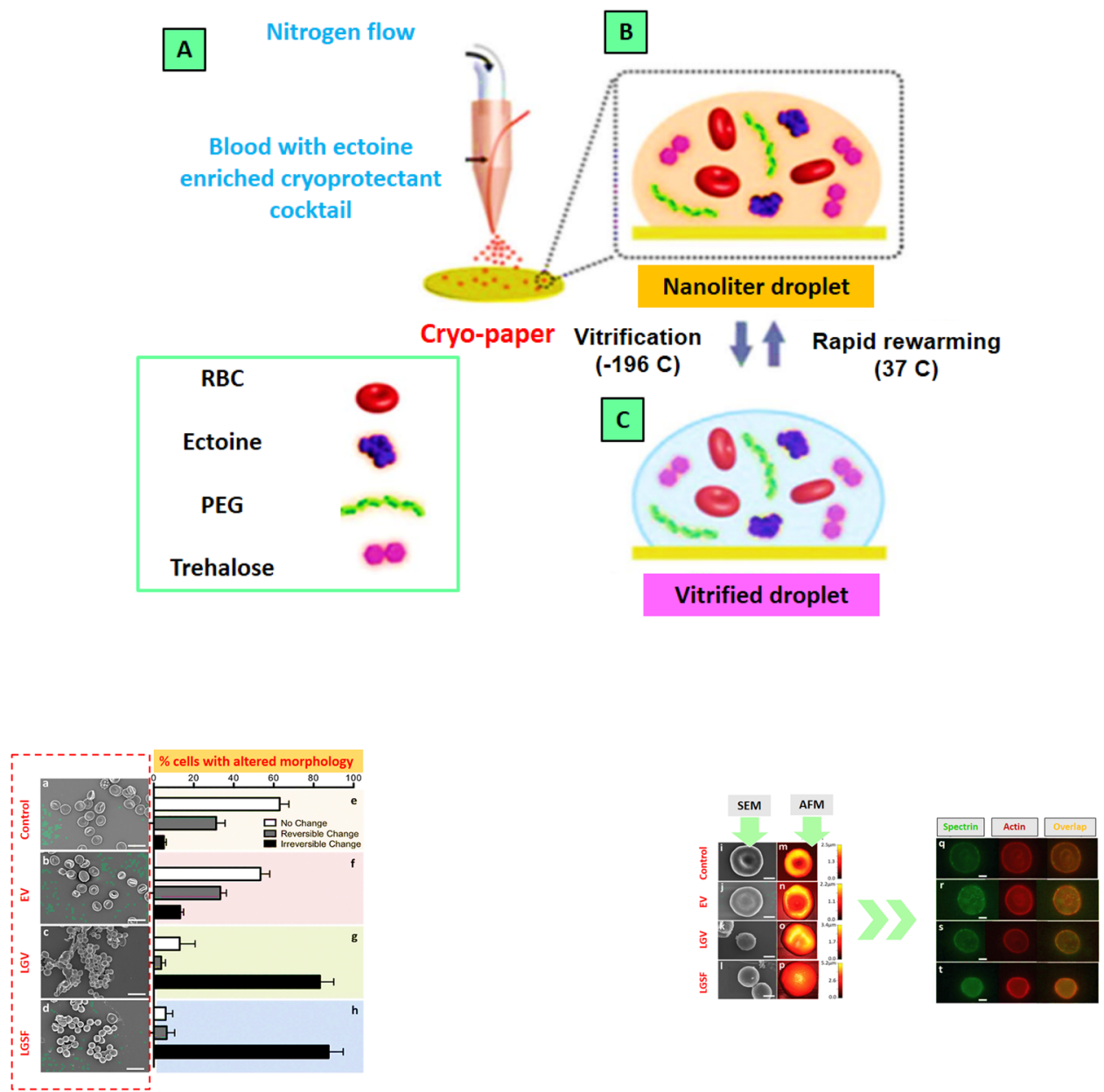

FIGURE $3{ }^{*}$ Droplet-based vitrification of red blood cells. (A) The essentials of the experimental setup for the droplet formation. (B) Schematic magnified view of a single droplet on the cryo-paper including RBCs, ectoine, trehalose, and PEG. (C) Schematic view of the vitrified droplet in (B). ${ }^{* *}$ Assessment of RBC morphology following ectoine-based vitrification and rewarding. (a-d) Scanning electron micrographs of RBCs: (a) selected directly from blood, and recovered after: (b) ectoine-based vitrification (EV), (c) low-glycerol vitrification (LGV), and, (d) low-glycerol slow freezing (LGSF). (e-h) The percentage (\%) of RBCs within a population representing: (e) untreated fresh blood, and blood recovered from: (f) EV, (g) LGV, and, (h) LGSF: biconcave shape, and reversible and irreversible shape changes ( $n$, number of cells $=114-$ 309 , and $N$, number of donors $=3-9$ ). Error bars in the figures represent the standard error of the mean. (i-l) Higher magnification (30,000 $\times$ ) of SEMs of: (i) fresh RBCs, and RBCs recovered after: (j) EV, (k) LGV, and, (I) LGSF. (m-p) Atomic force micrographs of: (m) fresh RBCs, and RBCs recovered after: (n) EV, (o) LGV, and, (p) LGSF. (q-t) Fluorescence micrographs showing distribution of the spectrin-actin network in RBCs. (q) RBCs in fresh blood, and RBCs recovered from: (r) EV, (s) LGV, and (t) LGSF. Scale bar represents $10 \mu \mathrm{m}$ in (a-d) and $2 \mu \mathrm{m}$ in (i-l) and (q-t).

\section{1 | Dry preservation}

One of the alternatives to cryopreservation is to skip the lowtemperature conditions and ship desiccating cells that can be stored at ambient temperature. It would be a cost-effective and practically good alternative for the long-term shipping of cells (Coopman, 2013). Freeze-drying (lyophilization) is helpful for the preservation of bacteria and fungi. Concisely, the process includes three steps: freezing of solvent, a primary drying phase for the sublimation of the resultant ice, and a secondary drying phase for removing the remained moisture by desorption (Sundaramurthi \& Suryanarayanan, 2012; Wolkers et al., 2002). During cryopreservation, cells can be pretreated and indeed lyophilized by lyoprotectants to minimize cell damage. Trehalose, a hydrophilic disaccharide that can be stored in desiccation-tolerant organisms, is a well-known compound detected (Crowe et al., 2005). According to Watanabe et al. (2016) studies, Pv11 was the first drypreservable animal cell line that showed improved survival and proliferation after dry storage. The method is mentioned in Figure 6, including preincubation, desiccation, and rehydration processes. As a result, they obtained a winsome drying and storage strategy containing trehalose preincubation for Pv11 cells at ambient temperature for $48 \mathrm{~h}$. They finally attained a favored dry preservation process for Pv11 cells. They prolonged this dry storage up to 251 days, and the cells still showed $7 \%$ viability (Watanabe et al., 2016). 
(a)

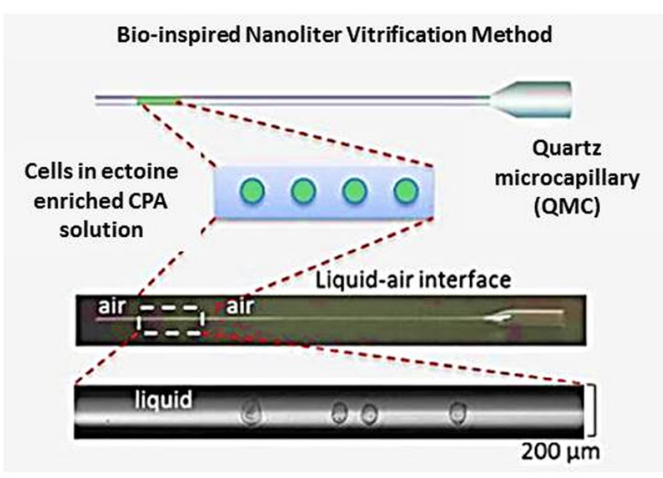

(b)

Dextran

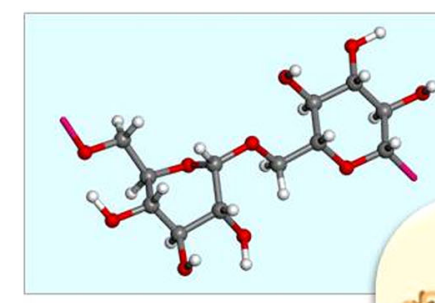

(c)

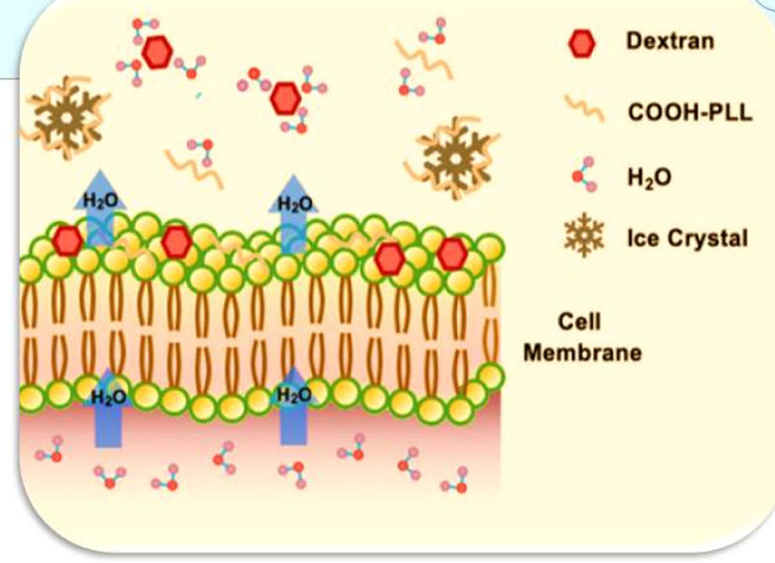

Carboxylated Poly-L-lysine (COOH-PLL)

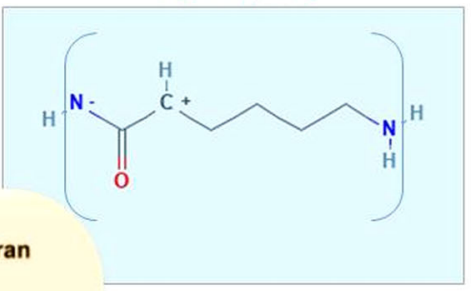

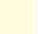<smiles>C1CCC1</smiles> 
Different Cell Line Shipping Methods

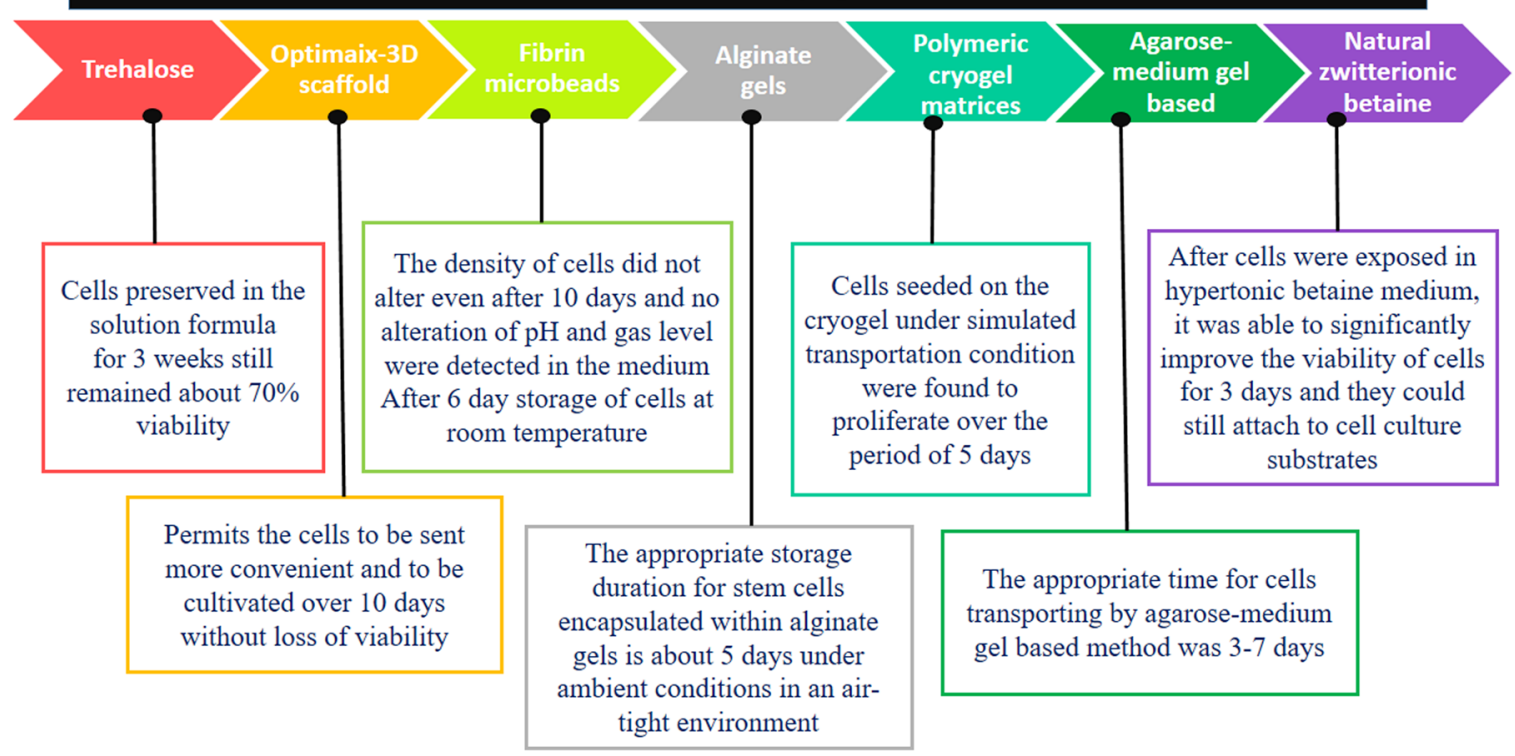

FIGURE 5 Storage time in different types of cell shipping methods

\section{2 | Hypothermic preservation}

Hypothermic preservation is the pausing of cells at hypothermic temperatures $\left(1-35^{\circ} \mathrm{C}\right.$; J. Yang et al., 2017). It is another potential strategy to be an alternative for cryopreservation. In this process, cells are kept at low temperatures (not freezing temperatures). It can slow down the cell metabolism in the absence of ice formation side effects. Cell growth is still observed under mild hypothermia $\left(25-35^{\circ} \mathrm{C}\right)$, and the expression of the recombinant protein may be enhanced by cell culturing at low temperatures (Miyamoto et al., 2009). More severe hypothermia and refrigerated storage $\left(0-10^{\circ} \mathrm{C}\right)$ are required to stop cell proliferation. It would be desirable to store cells in liquid nitrogen or apply dry shippers. This is a shortterm preservation method and is applicable to the pharmaceutical industry. It allows researchers to store cells for several days if a piece of equipment is double-booked or breaks down, rather than having to dispose of plates of cells, saving time and money (Coopman, 2013; Wise et al., 2009). Taken together, low-cost and simple methods have been developed to improve hypothermic preservation by the considerable potential of extending the shelf life of cell therapy products stored hypothermically (Swioklo et al., 2016).

\subsection{Gel-based methods}

\subsection{1 | Agarose-based hydrogel}

In the condition of cell transportation, agarose was widely used to determine the number of plaque-forming units in virology at a concentration of $0.8 \%$. Rigidity may be the most important factor in supporting adherent cells and preventing cell death. In light of this, the agarose-medium gel must have appropriate stiffness. Temperature adjustment is one of the important requirements for the application of the agarose-medium gel method. It should be between $1^{\circ} \mathrm{C}$ and $37^{\circ} \mathrm{C}$ since more significant than $37^{\circ} \mathrm{C}$ or lower than $0^{\circ} \mathrm{C}$ can be damageable for cells. On the other hand, the agarose concentration should be adjusted between $0.8 \%$ and $1.2 \%$. Because the agarose layer at a concentration of less than $0.8 \%$ is not hard enough, and at a concentration of more than $1.2 \%$, it becomes dry and fragile. The higher concentrations of agarose (higher than 1.2\%) might be the reason for low cell viability. The ideal condition for this method was to contain an agarose concentration of $1 \%$. In this way, it showed the highest level of cell recovery viability and also had enough rigidity to form an appropriate layer of gel and be protective until the shipping was over.

According to L. Yang et al.'s (2009) study on the agarose gelbased method, the numbers of plaque-forming units were detected for testing the viability and function of cells after transportation. Cell morphology after being kept in a medium containing agarose gel at room temperature was similar to that in normal cell culture conditions. Using this method, detection of colony formation revealed that the appearance of cell colonies was unaffected by transportation recovery (L. Yang et al., 2009). Wheatley and Wheatley reported a gel-based approach that allowed for up to 2 weeks of shipment without affecting post-transit cell recovery. Its application will help scientists and permit them to send cultured cells across the world without requiring dry ice and at ambient temperatures. In this study, a favored recovery is shown when cells are transferred at a concentration of $5 \times 10^{6}$ cells $/ \mathrm{ml}$ between $4^{\circ} \mathrm{C}$ and $27^{\circ} \mathrm{C}$ for 1 week. Before seeding, they were instructed to diffuse the gel pellet and ship cells in microfuge tubes (Wheatley \& Wheatley, 2019). 


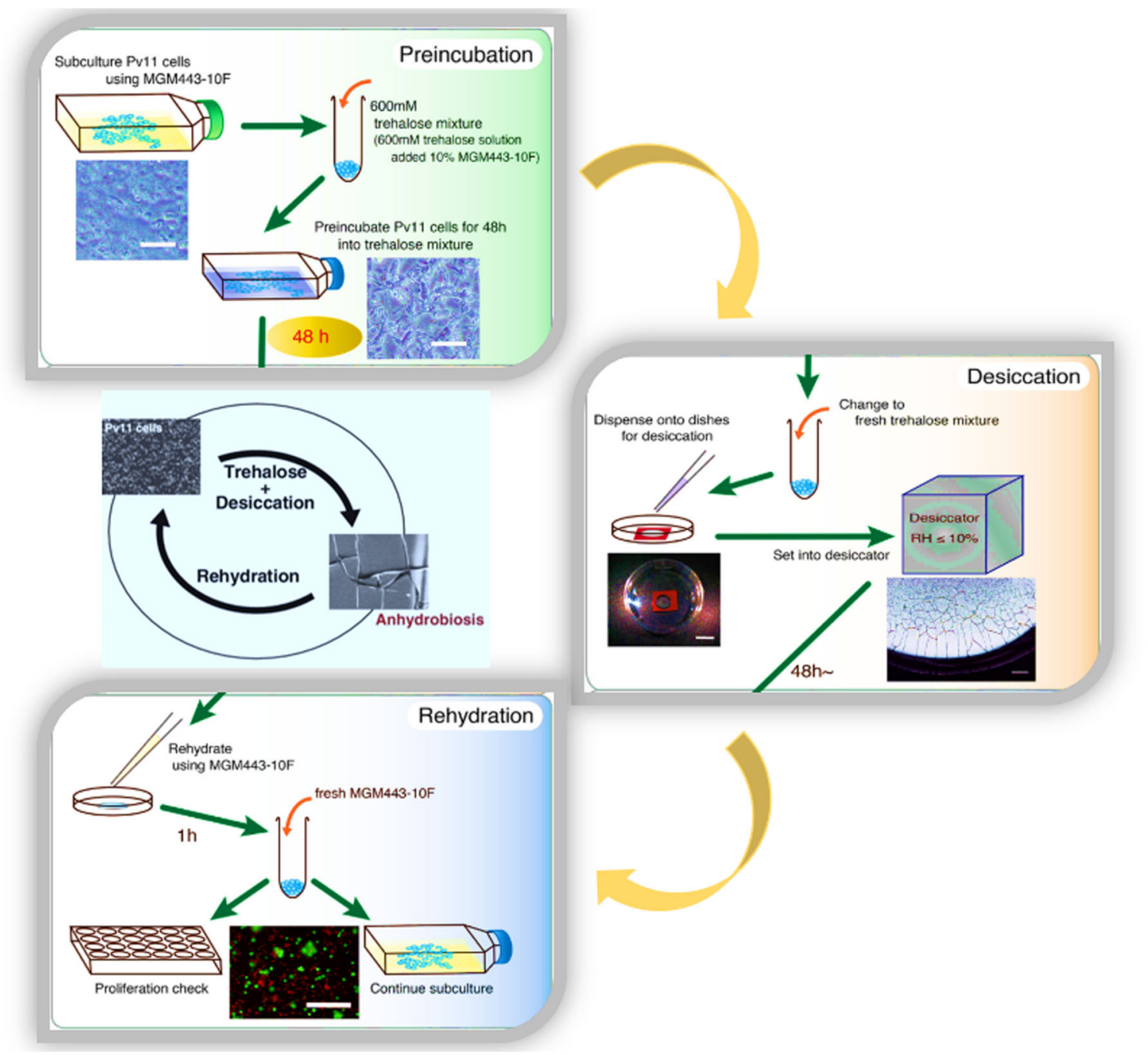

FIGURE 6 Schematic protocol for dry-preservation of Pv11 cells. Preincubation step: Pv11 cells were collected by centrifugation and resuspended into a $600-\mathrm{mM}$ preincubation trehalose mixture. The cell suspension was then incubated in a culture flask for $48 \mathrm{~h}$ at $25^{\circ} \mathrm{C}$. Pictures show the aspect of Pv11 cells before and after preincubation.

\subsection{2 | Gelatin-based hydrogel}

Stefansson et al. (2017) formulated a HemSol ${ }^{T M}$ gelatin reagent which could be a suitable replacement for dry ice shipping. It is possible to ship cells that require a surface to grow or for others that can be grown free-floating at ambient temperature in gel form. This form is not subject to airline regulations. This reagent comprised an FDAapproved patented mixture of sugars and gelatin. Cells can be kept in a multi-well plate, packed with $\mathrm{HemSol}^{\mathrm{TM}}$ gel, and sent to the receiving lab. According to Stefansson et al. (2017) studies, HemSol ${ }^{\mathrm{TM}}$ gel can protect platelet and red blood cells function in cold storage for up to 6 days. They also analyzed live cells' function during transportation with the HemSol ${ }^{\mathrm{TM}}$ gel method. The receivers will have cells with proper function and already plate to carry out the assays. As a result, $\mathrm{HemSol}^{\mathrm{TM}}$ gel is a distinct alternative to the dry ice method and has the potential to advance the pharmaceutical and biotechnological applications of cells in research. The cells shipped in
$\mathrm{HemSol}^{\mathrm{TM}}$ gel represented more than $95 \%$ viability and $2 \mathrm{~h}$ of posttransit cell recovery. Cells sent in dry ice required more than $24 \mathrm{~h}$ to recover their biological activity. Indeed, they required changing media to remove the DMSO (Steingrimur et al., 2017).

\subsection{3 | Alginate-based hydrogel}

Recently the frequent application of hydrogels in the role of carriers and delivers of cells is now known to compete with conventional cryopreservation methods. Hydrogels such as alginate, poly (lacticco-glycolic acid)/poly (L-lactic acid) scaffolds, hyaluronic acid, agarose, and chitosan have been used in hESC culture. Stem cells in suspension cell culture do not survive long periods of transportation at room temperature (Reddig \& Juliano, 2005). It required an appropriate delivery time as well as a reasonable financial cost. Hence, cell cryo-preservation was already known as invalid owing to 
the adverse effects of DMSO (Chakrabarti \& Clarence, 2001). Thus, suitable conditions are demanded for the preservation of stem cells. A biocompatible, chemically inactive, and structurally homogeneous hydrogel could be used as a simple and inexpensive storage method. It will subject stem cells to low manipulation and preserve their viability and functionality (B. Chen et al., 2013).

The cell samples used to study alginate hydrogels as cell carriers were human mesenchymal stem cells (hMSCs) and mouse embryonic stem cells (mESCs). These cells were properly kept inside the alginate hydrogels for 5 days at ambient temperatures in sealed cryo-vials. After removing alginate gel, cell viability results show $74 \%$ survival rates for $\mathrm{mESC}$ and $80 \%$ for hMSC, compared with survival rate results gained following cryopreservation. Indeed, the proliferation rate and detection of protein levels in hMSC and mESC extracted from alginate hydrogels were also comparable with cryopreservation (B. Chen et al., 2013). The mechanical features of alginate hydrogels are mainly related to the ratio of components used for gelation, including polysaccharides and the cationic cross-linker. Although calcium is the typical cross-linking ion for alginate gels, cations such as strontium and barium are more appropriate (Mørch et al., 2006; Place et al., 2011). Strontium-alginate microbeads were more steady and firmed than calcium alginate beads (K. L. Chen et al., 2007). The mechanical properties of alginate gels can also be controlled by balancing the internal porosity of these gels. The mixture of hydroxyethyl cellulose with alginate led to providing gels with controllable pore size, which was related to a better rate of cell viability (Wright et al., 2012).

\subsection{4 | 2-hydroxyethyl methacrylate-agarose and gelatin-based cryogel}

Recently, matrices provided at subzero temperature using cryogelation technology were known as cryogel. Cryogels with macroporous structures can be recognized as sponge-like hydrogels created based on cryogenic conditions and at temperatures below the freezing degree of water molecules (Katsen-Globa et al., 2014; Vrana et al., 2012). The polymeric cryogels have a 3D structure and were already attended as a scaffold for the tissue engineering field (Hanna \& Hubel, 2009). Cell-scaffold construct for regenerative medicine was cultured in vitro and then transplanted to in vivo (Sarkar \& Kumar, 2016; Umemura et al., 2011). Storing of the provided tissueengineered construct is prohibited within this process. Thus, the cryopreservation of cell-scaffold construct possessing viable and functional cells is a more advisable strategy to satisfy the demand. Their ready-to-use nature led to their fast utilization. These cryogelbased methods for cryopreservation have superiorities over the gelbased method because of no concurrency between cell encapsulation and gel formation (Vrana et al., 2012).

Cryogels were more desirable among the other gel carriers in bio chromatography and biomedical sciences. These matrices approximately simulate the $3 D$ structure of the native tissue extracellular matrix. Indeed, cryogels stability introduces them as excellent polymeric materials suitable for the construction of scaffolds in tissue engineering and targeted drug delivery investigations. Cryogels are known as gel matrices produced by polymerization at subzero temperatures. Ice crystals participate as pore-forming units whenever polymerization occurs at the unfrozen point. Figure 7 represents the schematic view of cryogel formation, and Figure 8 shows the physical characterization, digital image, and SEM image of 2-hydroxyethyl methacrylate (HEMA)-agarose (HA) cryogel, and gelatin cryogels (Bakhshpour et al., 2019).

Bioreactors are specified as devices that protect biological reactions. They possess a significant role in cell cultivation, temperature, oxygen, $\mathrm{pH}$ monitoring, and nutrient analysis. The bioreactors can be used to provide new functional tissue during the in vitro tissue engineering with the yield of regulatory signals expression. In several research publications, it has been mentioned that cryogels have a vital function in bioreactor platforms. The transportation ability of both HEMA-agarose (HA) and gelatin cryogels were reported recently. Also, these cryogels were suitable for in vitro and in vivo cell culture studies. In these conditions, they influence the improvement of cell morphology and enhanced mechanical stability (Bakhshpour et al., 2019). SEM analysis of both cryogels was performed for surface morphological analysis. The homogenous pore distribution was observed under SEM. The presence of pores is associated with the easy recovery of cells. Hence, pore walls provide adherent surfaces to the cells and help with cell growth (Kumari \& Kumar, 2017).

Kumari and Kumar (2017) have tested the chance of cryogel utilization during cell transportation. According to this study, the methodology of using cryogel for cryopreservation has been proved entirely. Cells received by the cryogel were functional and viable following the transportation based on HEMA-agarose (HA) cryogel. It has also indicated that the method based on gelatin cryogel not alter cell morphology and remained fit for cryopreservation even after 5 days. Cryogels not only control the transportation problems but also bring a ready-to-use scaffold for the engineering adherent cells. The application of cryogels for cell transport shows another advantage, such as an appropriate cell proliferation during transportation (Kumari \& Kumar, 2017).

In summary, HA and gelatin cryogels have the potential to transport and store adherent cells. These cryogels possess several surface properties and can be utilized for different applications. Kumari and Kumar (2017) produced HA cryogel for cell transportation with the chance of cells that could be eluted following the shipping. On the other side, they suggested gelatin cryogel utilized for cell shipping and cryopreservation. In both, cells were alive and had the capability of proliferation after simulated transportation. The adherent cells stored in HA cryogels and $\mathrm{C} 2 \mathrm{C} 12$ cells showed up to $25 \%$ more viability than the cells stored in cryovial under the same transport conditions. It is demonstrated that the adherent cells can be kept in gelatin cryogels and $\mathrm{C} 2 \mathrm{C} 12$ cells until 5 days at ambient temperature and represent the viability of $80 \%$. It is believed that cryogels could decrease the effect of shear force, which is the leading cause of the low viability in cell transportation. Cryogels act as a well- 

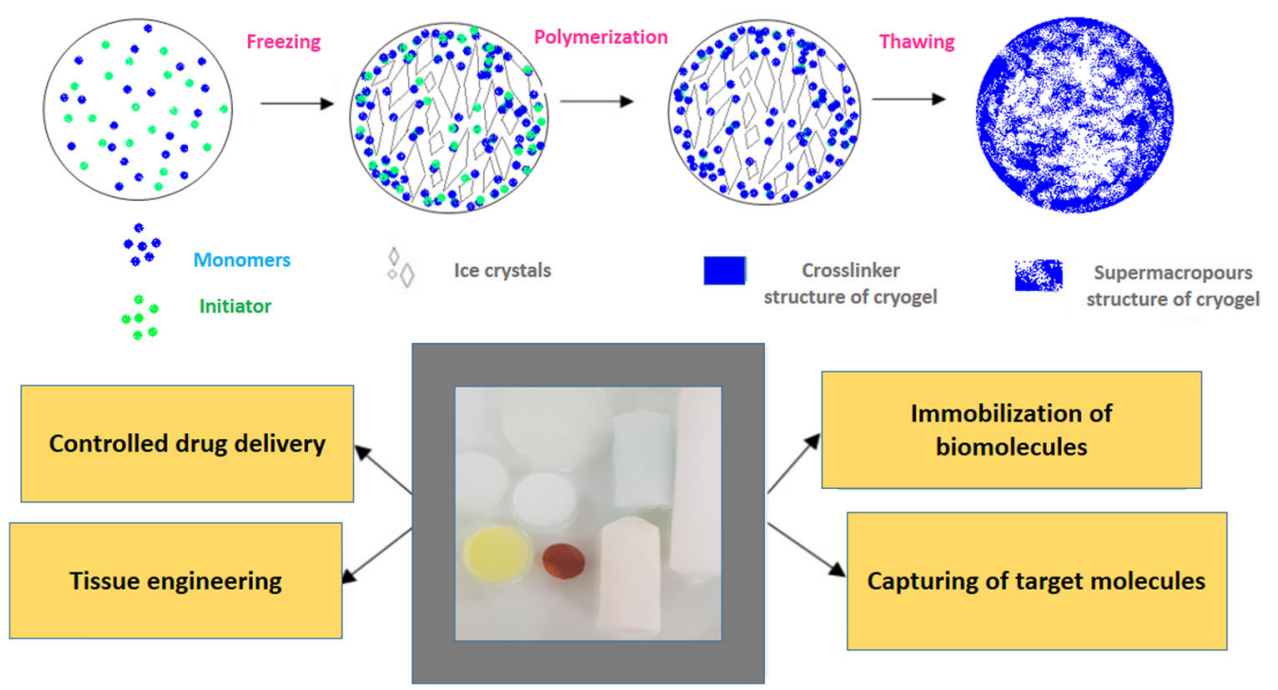

FIGURE 7 Schematic view of cryogel formation

\section{A}

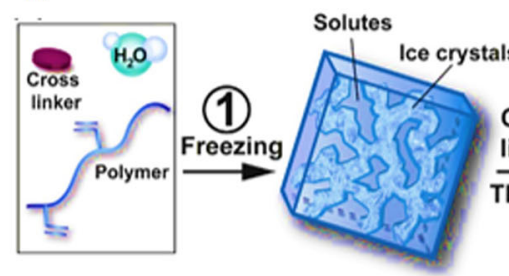

B
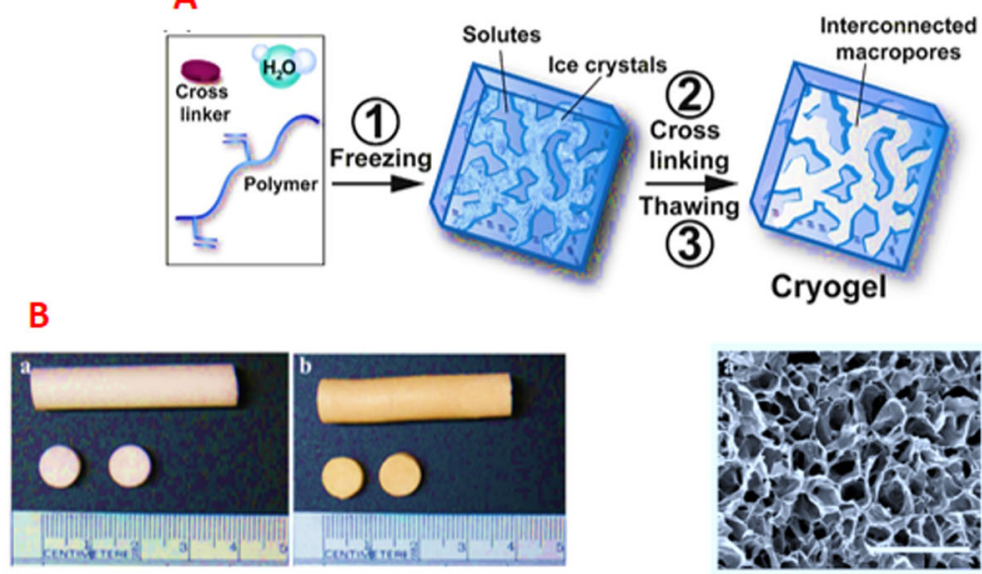

C

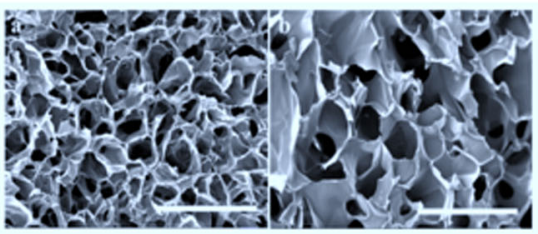

FIGURE 8 (A) For the formation of cryogels, (1) a hydrogel precursor solution is frozen $\left(T<0^{\circ} \mathrm{C}\right)$. This process leads to a phase separation of the solvent (e.g., water) into a frozen phase (ice crystals) and a nonfrozen phase around ice crystals where the gel precursors (monomers, polymer, crosslinker, and initiator) are expelled. (2) Next, concentrated gel precursors are crosslinked around ice crystals (porogens). (3) Following cryogelation, thawed ice crystals give rise to a macroporous sponge-like hydrogel, known as a cryogel. (B) Digital image of (a) HA cryogels and (b) gelatin cryogels. (C) Scanning Electron Microscopy image of (a) HA cryogels and (b) gelatin cryogels.

organized and cost-effective transporting matrix at room temperature and cryo-conditions. Hence, it may show serious outcomes for ready-to-use transplantation and regenerative medicines (Kumari \& Kumar, 2017).

According to some investigations, transportation of mammalian cells for long distances at ambient temperature can be performed through agarose gel-or matrigel-based methods. Also, it is shown that these procedures retain an acceptable cell recovery after a few days of shipment. These procedures are complex and labor-consuming. Whether mammalian cells can ship in an easy way but at ambient temperatures has remained obscure (Wang et al., 2015; L. Yang et al., 2009). The novel polymer-based cryogel matrix method is uncomplicated and affordable because specific devices and commercial mediums aren't needed. In this method, cells were directly suspended in the culture medium. It can be used for cell shipping at short and long distances. However, it should be performed carefully and cautiously during the transportation for clinical purposes where specific equipment is required (Wille et al., 2014). It not only expands our understanding of cell reactions to hypothermia conditions but also provides a new strategy for cell shipping. According to this method, the process for cell shipment would be helpful to biological and biochemical labs (Wang et al., 2017).

\subsection{5 | Collagen-based cryogel}

Cells interact with scaffolds via chemical ligands on their surfaces. Natural extracellular substances, such as collagen, are the precursor 
of scaffolds. The ligands of Arg-Gly-Asp have been identified naturally on the surface of the substance. The size of the pores present on the scaffold shows an essential role in cell attachment, survival, and function (Daei-farshbaf et al., 2014; Ranucci et al., 2000). Porosity, water-uptake capacity, permeability, and stiffness of the scaffold are also vital factors for cell-scaffold interactions. Ruoß et al. (2018) shipped cells on a scaffold over 10 days after characterization of the scaffold. Even though scaffolds have to be reproducible, Optimaix-3D is the name of a scaffold that is manufactured in a standard freezing process from a specified collagen dispersion (Schoof et al., 2001). Primary human hepatocytes need the interaction with extracellular matrix components (ECMs) to control their vital functions. Collagen, as an ECM protein, presents a significant role in maintaining tissues (Baiocchini et al., 2016). So, it is known as a biodegradable material utilized in medical and bioengineering sciences (C. H. Lee et al., 2001). Collagen is known to be the vital ECM protein of the healthy liver and possesses excellent biocompatibility, high porosity, and permeability. Therefore, the collagen used for the scaffold could be an appropriate carrier for primary human hepatocytes for long-term transportation and, or metabolic studies (Khan \& Khan, 2013). According to Ruoß et al. (2018) studies, the viability of the cell can be controlled by the Optimaix-3D Scaffold-based method compared to the conventional shipping method. Additionally, the utilization of Optimaix-3D Scaffold led to the maintenance of the essential hepatic functions and metabolism over 10 days (Ruoß et al., 2018).

The osmotic stress, cell-junction, cell transport systems problems, and disruption of organelles within the slow freezing method are stressful for cells. Kim et al. (2004) evaluated the effects of a freezing medium containing collagen on the proliferation of hESCs following the slow freezing and rapid thawing. Further tests were conducted to see whether 3D cultivation allowed cells to be shipped more easily and to be grown for 10 days without losing viability. Hence, the effect of the scaffold cultivation on critical metabolic functions of Primary human hepatocytes was tested for up to 10 days and compared to traditional 2D cultures. The basal urea production, one of the critical metabolic functions of primary human hepatocytes cultured on Optimaix-3D scaffolds, was about $50 \%$ higher than primary human hepatocytes cultured in 2D. Hence, essential urea production of primary human hepatocytes in 2D cultures decreased quickly within 10 days. On the contrary, the urea production in 3D cultures remained stable over 10 days. It was reported that Optimaix-3D cultures produced more albumin than 2D cultures (Ruoß et al., 2018).

\subsection{Encapsulation methods}

\subsection{1 | Alginate microcapsules}

Cell microencapsulation technology is a crucial technique for prevailing over the bioprocess challenges. Alginate microencapsulation of cells is a unified bioprocess for cryopreservation of pluripotent hESCs. The presence of EMCs in microcarrier cultures may help cells survive freezing and thawing by reducing post-thaw apoptosis (Ji et al., 2004; Kim et al., 2004). The alginate microcapsule provides an extra rise in post-thaw cell viability of up to three times greater compared to unmanipulated cultures. The related mechanisms are still obscure, but several experiments demonstrated that controlling the cell-cell/matrix contacts could improve the hESC recovery following cryopreservation (Hunt, 2007; Karlsson \& Toner, 1996). Alginate microencapsulation of cells may help hESCs against cryopreservation risks, including disruption of cell-cell and cell-matrix contacts and intracellular ice formation (Malpique et al., 2010; Zimmermann et al., 2005).

Alginate is one of the common encapsulation materials owing to its biocompatibility, biosafety, and permeability. Nie et al. reported a novel technique for the cryopreservation of hESCs by microcarriers. Still, this procedure required more optimization to omit animal feeder cells and enhance cell survival after thawing (Serra et al., 2011). According to the investigation performed by Swioklo et al. (2016), the alginate-encapsulation method was applied to improve the traditional preservation of human adipose-derived stem cells. They evaluated the essential conditions required for hASCs cell function during the hypothermic storage. Due to inert hydrogel characterization of alginate, cells were separated (having little or no physical cell-tocell interaction) which can lead to the protection at higher temperatures. After $24 \mathrm{~h}$ of encapsulation in culture, it was not detected any alterations in the metabolism of cells. The cytoprotection was maintained in cell densities, and the result was $70 \%$ viability. Assessment of cell morphology following the storage showed that growth kinetics had not changed in all samples until Day 6. In this study, it was demonstrated that cell density affects viable cell recovery (Stacey et al., 2017; Swioklo et al., 2016).

The appropriate storage duration for encapsulation of cells by alginate gels is about 5 days at ambient temperature in an airtight environment. A novel and easy utilization of alginate hydrogel encapsulation will provide an inexpensive and powerful alternative to cryo-preservation for cell transportation in cellular studies. This method also allows easy cell recovery to retain their viability and phenotype (B. Chen et al., 2013). The biocompatibility and rapid gelation are the significant properties of alginate-based cell encapsulation that provide the success of cell encapsulation. Agitation might be the cause of encapsulated cell death, because the calcium alginate gels are incredibly fragile. It was known that strontium cross-linking with alginate gels was an excellent way to solve this problem because it would stop the alginate hydrogel from fragmentation during longdistance transport. Strontium showed a high affinity to alginate compared to calcium, which led to stability improvement of alginate hydrogels. It was reported that strontium alginate hydrogels were a successful method in bone tissue engineering. B. Chen et al. (2013) increased the gels' mechanical power by an inert nylon mesh encapsulation. The mixture of strontium alginate gel and nylon mesh provided an enormously effective carrier for hMSC and mESC. Both $\mathrm{mESC}$ and hMSC markers were optimistic following the extraction even after 5 days and remained at ambient temperature (B. Chen et al., 2013). 


\subsection{Fibrin microbeads}

Fibrin microbeads (FMBs) generated from human fibrin are nonimmunogenic, biodegradable at a slow rate, and have extended shelf life; they are employed as cell carriers. FMB is easy to use and worthy equipment for MSCs isolation from mixed sources. Also, it was considered to show potent application in cell-based regenerative medicine and support the expansion of many matrix-dependent cells in a 3D culture. The density of cells produced with FMB and cultured in suspension under 3D conditions increased (Ben-Ari et al., 2009; Gorodetsky et al., 1999; Shimony et al., 2006). Other biodegradable microspheres and microcarriers have been proposed for 3D cell culture (Chung \& Park, 2009; Kang et al., 2008; Park et al., 2011), but they lack multifunctional properties and do not simultaneously separate stem cells and preserve them during differentiation. They act as the carrier for cell implantation. According to one study, the FMB-based technique was used to deliver mesenchymal cells and other matrix-dependent cells in sealed vials at room temperature for a long time transportation of $>10$ days. They reported excellent cell survival without any need for further supportive infrastructure. The mechanism of MSC attachment to FMB by hypoxia-induced factor has been studied by Gorodetsky et al. (2011). They tested the rate of proliferation of the cells after room temperature storage. They reported that after recovery time, cells kept their properties as before and after 6 days of room temperature storage. The results showed that the cells remained alive and showed the same proliferation rate. Figure 9 represents the setup of cells cultured on FMBs. The density of cells sealed on FMBs at room temperature did not alter even after 10 days. After 6 days of cell preservation at room temperature on FMBs, no change in $\mathrm{pH}$ or gas level was identified in the media. FMBs are types of cell carriers that could help transfer progenitor cells at room temperature for long-term intervals between different research centers (Gorodetsky et al., 2011).

\section{6 | Osmolyte solution compositions}

Natural osmolytes such as sugars, sugar alcohols, and amino acids help maintain the stability of biological systems subjected to harsh environmental conditions. Raman images illuminate the interactions between osmolytes and water by depicting the effect of osmolytes on the morphology of ice crystals. Pi et al. characterized the freezing reactions of several human $\mathrm{PBMC}$ and specific white blood cell subsets within them using combinations of osmolytes, including SGI (sucrose-glycerol-isoleucine), TGI (trehalose-glycerol-isoleucine), and MGI (sucrose-glycerol-isoleucine) (maltose-glycerol-isoleucine). T cells (CD3+ CD56) and $T$ cell subsets such as helper $T$ cells (CD3+ CD4+) and cytotoxic T cells (CD3+ CD8+) are evaluated the following thawing. They previously studied the effect of mixing osmolytes on preservation quality and the cell-osmolytes interaction in Jurkat cells. They discovered that sugars, sugar alcohols, and amino acids in multicomponent osmolyte solutions were beneficial for cell cryopreservation ( $\mathrm{Pi}$ et al., 2019, 2020). They also defined the "sweet spot" for preserving a T-cell line using osmolytes in the other investigation (Pi et al., 2018).

The thermophysical properties of each cryoprotectant solution were evaluated. The melting enthalpy of the trehalose-glycerolisoleucine formulation was the lowest, indicating that less ice was generated. While this formulation was not optimum for all cell types, it demonstrated that the mass of ice formed during the freezing process is not the only factor affecting cell recovery. Biology has a role in the cryopreservation process. The findings suggest that the biological interactions between cryoprotectants and water as well as between cryoprotectants and cells, may contribute to an improvement in the recovery of sensitive cell types. These cell types respond differently to cryoprotectants, and it is possible that no single cryoprotectant can maximize all branches simultaneously. Finally, knowledge of the freezing reactions of various subsets of PBMCs will (a)

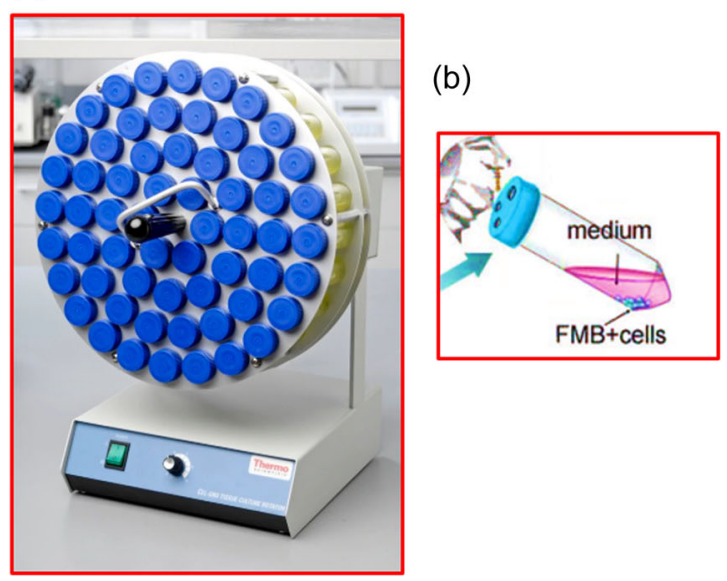

(c)

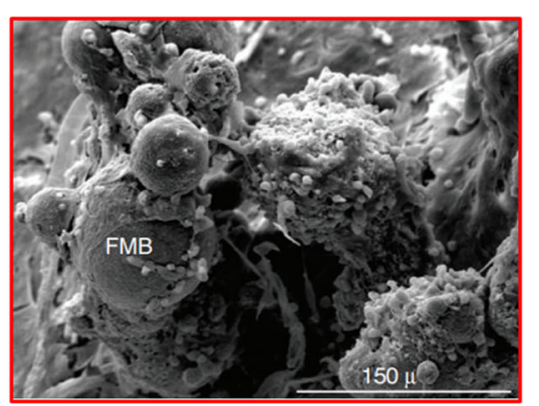

FIGURE 9 The setup of cells cultured on fibrin microbeads (FMBs) in regular tubes placed in a slow rotator in a $\mathrm{CO}_{2}$ incubator (a). The cells grown in special tubes with a covered perforated cover for air exchange with preservation of sterility, as shown in (b). Attached cells on the surface of the FMB are shown in (b) by electron scanning microscopy (c). 
enable more effective and successful cryopreservation ( $P$ et al., 2020).

\subsection{1 | Trehalose-contained solution formula}

Trehalose is a glucose disaccharide capable of stabilizing and preserving cells or tissues during freeze-thaw operations (Erdag et al., 2002). Although the mechanisms were unknown, they might be employed to replace CPA to cryopreserve red blood cells and germ cells (Eroglu et al., 2002; Satpathy et al., 2004). Few studies have been conducted to assess trehalose's capacity to protect cells from apoptosis at relatively high temperatures $\left(48^{\circ} \mathrm{C}\right)$. A study was conducted to determine the influence of trehalose on the survival of MSCs at $48^{\circ} \mathrm{C}$. Di et al. (2012) devised a solution formula capable of effectively preserving MSCs for up to $2-3$ weeks at $48^{\circ} \mathrm{C}$.

Trehalose is a nontoxic substance that has been extensively utilized as a natural CPA for cell cryopreservation and hypothermic storage of human tissues (Erdag et al., 2002; Pu et al., 2005). Due to its safety, trehalose does not need to be eliminated from cells before use in research or clinical settings (Samantha \& He, 2018). It has been demonstrated that the addition of trehalose to cryopreservation solution dramatically enhances the survival of human hepatocytes and amniotic fluid-derived stem cells (Katenz et al., 2007; Seo et al., 2011). In addition, the low intracellular concentrations of trehalose may increase the survival rate of human cells after cryopreservation (Eroglu et al., 2000; Satpathy et al., 2004). However, the exact mechanism of trehalose effectively preserves biological systems during freezing and drying is unknown. It is hypothesized that trehalose stabilizes phospholipids and proteins by interacting directly with their polar groups throughout the freezethaw cycle (T. Chen et al., 2001; Crowe et al., 2003).

Cryoprotectants are classified into two types of penetrating and non-penetrating (McGann, 1978). The penetrating types comprise glycerol, 1, 2-propanediol, and DMSO. The non-penetrating cryoprotectants involve polyvinylpyrrolidone, trehalose, fructose, sucrose, and glucose. Nematodes and yeasts, for example, contain trehalose, which can tolerate freezing and drying. Human platelets and red blood cells, sperm, oocytes, pancreatic islets, and fetal skin have been cryopreserved using trehalose in prior studies. For cryopreservation of stem cells extracted from umbilical cord blood and bone marrow, trehalose has been reported as an alternate procedure. Also, in a study by Scheinkonig et al., cryopreservation of stem cells obtained from mobilized peripheral blood stem cells has been reported. In contrast, trehalose was employed in another study for cryopreservation of pure hematopoietic stem cells and progenitor cells (Beattie et al., 1997; Erdag et al., 2002; Eroglu et al., 2002; Martinetti et al., 2017; Motta et al., 2014; Scheinkönig et al., 2004; Wolkers et al., 2001; Zhang et al., 2003).

The anhydrobiotic compound trehalose (a, a-trehalose, a-Dglucopyranosyl-a-D-glucopyranoside) has been employed as a cryo/ lyoprotective agent with or without additional CPAs in a variety of therapeutic applications ranging from blood banks to reproductive technologies (Eroglu et al., 2000; Y.-A. Lee et al., 2013). In the case of a water shortage, trehalose forms hydrogen bonds with biomolecules and retains their functional conformations (Lawson et al., 2011). In fact, trehalose operates as an impermeable molecule, resulting in the creation of a persistent glassy matrix with limited molecular mobility. It suspends intracellular degradative metabolic processes in response to water loss by blocking the associated lipid phase shift (Hara et al., 2017).

Several research investigated the effect of adding relatively small amounts of DMSO and trehalose as CPAs on long-term cryopreservation (Beattie et al., 1997; Choi \& Chang, 2003; Yokomise et al., 1995). Pu et al. (2005) proposed a method of cryopreservation utilizing trehalose as a cryoprotectant. They developed a practical technique by adding trehalose alone as CPAs. It is regarded as a superior long-term preservation strategy for adipose tissues due to the vastly improved vitality of cells. Additional research is needed to fine-tune their cryopreservation approach using trehalose as a CPA. Due to trehalose's nontoxic properties, it may be employed alone, as a single CPA, in the long-term preservation of adipose tissues, and the stage of removal is irrelevant (Pu et al., 2005).

\subsection{2 | Sucrose-based media}

Several mammalian cell types have frequently been cryopreserved with sugars and other non-DMSO cryoprotectants in place of DMSO. According to study findings, carbohydrates can be efficient in cell preservation when combined with sugar alcohols and amino acids. Numerous research have been conducted to elucidate the processes underlying sucrose's protective benefits, the majority of which focused on the interactions of sucrose with water, protein, and membranes. Yu et al. (2018) studied the interactions between sucrose and water in a frozen sucrose solution. They found direct linkages between sucrose and the cell membrane at low temperatures, as well as the protective mechanisms of sucrose. In concentrated sucrose solution, a stronger hydrogen bond network was discovered. They hypothesized that the protective properties of sucrose are due to its direct interaction with cells. Using the high spatial resolution of Raman spectroscopy, scientists are recently able to visualize the distribution of extracellular and intracellular ice, cryoprotectants, and the size of ice crystals formed in various combinations of cryoprotectants during the freezing duration. They used Raman spectroscopic images to quantify the difference in sucrose content between a cell and the ice phase. At the interface of the cell and extracellular ice, the concentration of sucrose was lower than in nonfrozen solutions in bulk (Yu et al., 2018). Shanina et al. (2000) conducted a study comparing the preservation of hepatocyte cells using a sucrose-based solution to that of another preservation medium. According to their findings, a sucrose-based solution for cold preservation of isolated hepatocytes may be a less complicated option. The stability of liver cells at low temperatures was determined using the trypan blue dye exclusion method and their morphological appearance. In sucrose-based solutions, dye exclusion was significant than in other media (Shanina et al., 2000). 


\section{5 | DISCUSSION}

Mammalian cells must inevitably be transported internationally from one research location to another (Wang et al., 2017). According to some ancient cell shipping procedures, they require a particular container and either dry ice or medium to fill flasks of live cells. Some researchers send medium-filled cell culture flasks, although agitation might result in the detachment of cell monolayers. It is not appropriate for long-distance shipment (Nienow et al., 2016; Steingrimur et al., 2017; Suuronen et al., 2008; Wang et al., 2017). Because liquids constitute a spill risk, they are forbidden in postal and air transit (Steingrimur et al., 2017; Wheatley \& Wheatley, 2019). This also has a limited shipping time problem that cells in media have limited viability with flask leakage. It is only helpful in moving cells across a fixed distance in a short time (usually up to $24 \mathrm{~h}$ ). Additionally, prolonged storage of CPAs such as DMSO is hazardous to cells. Numerous cell types do not survive or develop well during transit in cell culture flasks due to the quick exhaustion of oxygen and pH shift (Wang et al., 2015). However, the current approach of using liquid nitrogen for the transfer of stem cells is technically challenging (B. Chen et al., 2013). Indeed, shipping of cells in liquid nitrogen is dangerous and can be prohibitively costly (Steingrimur et al., 2017). Recent investigations have examined innovative enhanced cryopreservation technologies and alternate ways for transporting live cells at ambient temperature without dry ice or in media-filled flasks. From a development standpoint, It is now possible to design a "ready to go" cell system for high throughput applications. These processes ensure that investigators have functional cells ready to undertake tests.

Although vitrification has several advantages over other cryopreservation methods and reduces the time exposure of ova and embryos at ambient temperature, it nevertheless indicates drawbacks and disadvantages (Campos-Chillòn et al., 2006). There are two significant concerns about vitrification, including the toxicity of the high concentration of cryoprotectants and microbial contamination of liquid nitrogen (Barun, 2015). Alternative approaches for optimizing the vitrification procedure have focused on improving the cooling rate. It may allow for lower cryoprotectant concentrations to attain a glassy state. Cells have been placed within thin open straws, cryotops, electron microscopy grids, or cryoloops to enable faster cooling rates. Recently, scientists have confirmed the use of quartz micro-capillaries for vitrification, which allowed for extremely rapid cooling rates and required significantly lower cryoprotectant concentrations than traditional vitrification procedures $(\sim 2.0 \mathrm{M}$; Criado et al., 2011; He et al., 2008; Kuwayama et al., 2005; H. J. Lee et al., 2009; Vajta et al., 1998). A vitrification protocol has been developed to overcome the restrictions of current cryopreservation protocols. It is easily standardized and applicable to a large variety of cell types. This approach utilizes fused silica micro capillaries that act as a low-thermal-mass, high-heat conductivity cell container to increase the cooling rate significantly. This method of vitrification is simple and much faster than traditional vitrification techniques (in less than 15 min; Heo et al., 2015).
According to previous studies, low temperature can decrease cell growth rate (Azambuja et al., 1998; Kaushik \& Kaur, 2005; Vergara et al., 2014), although modest heat stress increases cell proliferation and growth (Choudhery et al., 2015; Tounkara et al., 2012). Increasingly, long-term treatment at subzero temperatures can influence the viability rate of mammalian cells (Jin et al., 2008; Moussa et al., 2008; Wang et al., 2017). Since under in vitro conditions, one of the vital environmental factors for cell survival is temperature. A standard requirement for mammalian cells is to culture usually in the incubator, which in $\mathrm{CO}_{2}$ levels are set to $5 \%$ and temperature to $37^{\circ} \mathrm{C}$ unless a specific research purpose is required (Brown, 1990). As a result, it seems that the application of methods based on ambient temperature would be safer and more careful than using methods based on low temperatures. The time for transportation has a prominent role in choosing different kinds of cell shipping methods. In this review, we suggest numerous techniques for the various time requirements in the transportation process.

Live cells are delivered directly to a plate using the agarose-based hydrogel technique (e.g., six-well plate). This approach offers the following advantages: biocompatibility, biodegradability, minimal cytotoxicity, cheap cost, no particular container or dry ice required, and no liquid on the cell plate. Cells shipped in live conditions have a fast recovery process following the receiving time compared to traditional methods (L. Yang et al., 2009). However, natural hydrogels have several limitations; for instance, they lack strong mechanical qualities, and manipulation is challenging due to their substantial batch-to-batch variance. As a result, natural hydrogels are frequently mixed with synthetic ones to produce composite polymers that are extensively investigated. The drawback of using collagen as a biomaterial is that the degradation process will produce thrombogenic amino acids that trigger the coagulation cascade (Catoira et al., 2019).

Cell encapsulation technology has dramatically improved in recent years, but there are still some challenges and limitations for clinical applications. The systemic biocompatibility of encapsulated cells must be improved. Excessive production of reactive oxygen species, cell death, and necrosis may occur over an extended time. As a result, it is necessary to establish a high rate of cell survival to ensure cell transportation. Microcapsules are needed to keep the cells safe from the immunological reactions of the host. Another limitation is that capsule breakage occurs because of poor stability. Another restriction of this technology is the difficulty of manufacturing a single microcapsule for all cells (Zhang \& He, 2011). The main limitations of FMBs are their weak mechanical strength and rapid degradation rate of fibrin (Ahmad et al., 2015). However, these limitations can be improved by either mixing with other polymers or cross-linking methods. An appropriate immobilization of cells with growth factors and other biomolecules is required to accelerate and support cell proliferation (Rajangam \& An, 2013). Recent 3D scaffolds have limitations, as they are frequently incompatible with 96-well plates. As a result, these technologies are only marginally suitable for high-throughput approaches. However, because Optimaix-3D scaffold is ideal for $\mathrm{PHH}$ transfer and long-term functional culture, it may be employed in various standard plate sizes, including 96-well plates. This collagen scaffold has a well-organized pore 
structure, excellent permeability, and high flow rates as compared to prior cryogels described in the literature for the culture of various cell types. On the other hand, techniques for detaching cells from scaffolds should be improved, as it is difficult to remove cells from scaffolds without significant cell loss quantitatively (Ruoß et al., 2018).

Designing of a nontoxic, animal serum-free preservation medium is a critical problem for mesenchymal stem cell storage and dissemination. Trehalose, in particular, is effective in clinical studies using blood stem cells. Trehalose has been shown to increase cell viability during thawing compared to the normal freezing process. These data can confirm the potential of trehalose application for cell preservation studies in the future (Martinetti et al., 2017). Because the inflow or outflow of undesirable small molecules may be detrimental to the cells, techniques that enable rapid and selective trehalose absorption are favored. Intracellular trehalose concentrations could be raised by optimizing nanoparticle design, allowing for faster and effective cellular absorption (Samantha \& He, 2018). Indeed, the effect of mixing osmolytes on preservation quality showed that sugars, sugar alcohols, and amino acids could be used to cryopreserve cells (Pi et al., 2019, 2020). Numerous investigations have been conducted to elucidate the processes underlying sucrose's protective properties at low preservation temperatures. Raman spectroscopy images were used to quantify a fluctuation in the sucrose content associated with a gap between a cell and the ice phase. The sucrose content was lower at the cell-extracellular ice interface than in the bulk nonfrozen solution. Sucrose's protective capabilities may result from its close interaction with cells (Yu et al., 2018).

Regulatory requirements, including appropriate standards and controls, should consider for cell-based clinical therapies. For this approach, stem cell-based products must be safe, effective, and high quality. There is no specific guideline for stem cell-based products as mentioned in $\mathrm{ICH}$. According to the CBER, the division of the US FDA, those cell-based therapeutics for homologous use (not combined with a drug or device) with minimal manipulation, labeling, or advertising do not need FDA approval. For the clinical studies, IND (Investigational New Drug) exemption is required. In this way, explaining study protocols is necessary. To date, it has been required to strategically manage the timing and settings of cell transfer to cover the pivotal gaps in cell shipping methods and to enable the transfer of functioning live cells over an extended period of time. The current understanding of the response of mammalian cells to the novel cell shipping methods mentioned in this review is less studied. Ultimately, further investigations are required to fix these methods as a typical process that might broaden the use of cells in pharmaceutical and biotechnology applications. The impact and role of alternative CPAs have been well described. Still, gaps in our knowledge exist concerning the application of and the effect of novel methods of cell preservations in long time intervals.

\section{6 | CONCLUSION}

The cell metabolism almost wholly ceases at low temperatures (e.g., $-196^{\circ} \mathrm{C}$ ), resulting in unavoidable side effects, such as genetic drift caused by biological differences in lipids and proteins that may lead to impairment in cellular function and structure. If there were no restrictions on the amount of utilized CPA, cells would be preserved excellently (Jang et al., 2017). CPAs, however, can be toxic to cells in conventional and normal conditions, especially when utilized in high doses. For instance, DMSO may impair chromosomal integrity, increasing the chance of tumor growth. Potential cell infection or contamination should be avoided, aside from endogenous cell modifications (Jenkins et al., 2012; Yong et al., 2017). Cell membrane damage and apoptosis have long been connected with cryopreservation (Yong et al., 2017; Trusal et al., 1984). Additionally, cryopreservation is time-consuming and operator-dependent, and it frequently requires equipment that is not ideal for cell therapy, such as liquid nitrogen, which is not GMP compliant (B. Chen et al., 2013). While cryopreservation is undoubtedly the most remarkable approach for long-term preservation of stem cells at the moment, its drawbacks have prompted research into alternatives. The contents reviewed in this study illustrate ongoing interest in improving and modifying preservation methods.

Successful cell shipment requires nontoxic protective chemicals and a preservation technique that does not impair the cell's structure or functionality considerably. Additionally, ideal preserved cells would be immediately usable upon return to ultra-zero temperature, without the need to remove any agents or complete additional processing processes. To our knowledge, there is not any article focusing on this critical issue in cell biology and biotechnology. We should encourage for development of novel cell shipping methods to solve the problems of traditional ones. According to novel cell shipping methods studied in this review, we reached some conclusions about them. Based on the evaluation of the appropriate time for cells transporting in these methods, it is clear that cells preserved in the formula containing trehalose represented $70 \%$ viability for 3 weeks, and they exhibit similar growth kinetics, expression of cell surface antigens, and differentiation capability to freshly extracted mesenchymal stem cells. Afterward, the Optimaix-3D Scaffold enables cells to be delivered more conveniently and cultured for 10 days without losing viability. In the case of the FMBs containing method, the density of cells sealed on them at room temperature did not alter even after 10 days. There were no alterations in $\mathrm{pH}$ or gas level in the medium after 6 days of storage in the cells on FMBs at room temperature. Under ambient settings in an airtight environment, stem cells encapsulated inside alginate gels can be stored for about 5 days. The appropriate time for cells transporting by agarose medium gel-based method was 3 days (no more than 5 days). After 3 days of exposure to hypertonic betaine media, the vitality of the cells increased significantly, and they retained their capacity to adhere to cell culture substrates. Altogether, we concluded that the preservation of cells by a formula containing trehalose is considerably more convenient and has a longer acceptable storage time than the other approaches discussed in this review. Trehalose has previously used to cryopreserve human cells such as platelets, red blood cells, sperm, oocytes, pancreatic islets, and fetal skin. Trehalose has used in numerous therapeutic applications ranging from blood banks to reproductive technologies as a cryo/lyoprotective agent combined with or without other CPAs. 
Finding that trehalose contained solution formula can be appropriate for different types of human cell transportation makes it a nontoxic compound that can commonly use as natural CPA. Indeed it has been demonstrated that trehalose can be employed as a single CPA in preserving cells for extended periods. A few studies have investigated trehalose's ability to keep the cells safe from apoptosis in relatively high temperatures (i.e., $48^{\circ} \mathrm{C}$ ). It should be noted, however, that the appropriate method for cell shipment is cell line dependent, and it must be examined which method is the best one for different investigations. It is evident that various cell types respond differently to cryoprotectants, and there may not be a single cryoprotectant that can optimize all subsets simultaneously. However, understanding how various cell types respond to freezing will enable the development of more effective cryopreservation technologies. To overcome the challenges that have plagued bioengineering, this review proposed a novel approach for DMSO-free cryopreservation utilizing biocompatible materials. With further developments, the knowledge of offering an organic solvent and DMSO-free technique may improve the field of medical science by enabling the broad usage of live cells. There is an outstanding question in the field of cell preservation whether an effective method can be devised to facilitate the dissemination of the growing number of cell products.

The power of cell shipping at ambient temperatures led to the elimination of the barriers impeding scientific projects. The economic and logistical advantages of ambient temperature shipping can reduce some lab difficulties. Unfortunately, many investigators are still unaware of the possibility of ambient temperature shipping. Following the rapid development of novel and combination therapies, we suggest a more excellent selection and continued objective research for new CPA molecules, which may well be in demand in the future. The creation of novel cryoprotectants will necessitate the development of increasingly advanced high-throughput screening technologies capable of assessing the effectiveness and biological impacts of all cryoprotectants. Although the improvements in preservation methods are still on a small scale, they are worthy, when introducing methods that can be maintained at room temperature and avoid using DMSO. It remains to see how widely applicable these novel preservation methods are when the impact of preservation explored in more kinds of cells and large scale. Current methods of cell preservation are highly efficient, but they are operator-dependent. New preservation technology is needed to facilitate the processing of cells with high efficiency. Novel strategies need to be developed and provide more effective ways to preserve cells in different conditions. It is a new topic in cell biology therefore it will take a while to get a proper classification. Here we just aim to introduce and gather some novel strategies of cell shipping that can be an alternative to traditional methods. We are sure that with the advancement of cell biology, new articles will be published in the future with different classifications of cell shipping methods. As these products start scaling up, they are more open to new ideas. Imagine how easy it would be if we could ship cells at ambient temperature without worrying about high temperatures.

\section{AUTHOR CONTRIBUTIONS}

Shabnam Heydarzadeh and Mehdi Hedayati designed and drafted the manuscript, collected the references, and carried out the primary literature search. Seti Boroomand and Sima K. Kia modified the manuscript and participated in discussions. All authors read and approved the final manuscript.

\section{ACKNOWLEDGMENT}

The funding information is not available.

\section{CONFLICT OF INTEREST}

The authors declare no conflicts of interest.

\section{ORCID}

Shabnam Heydarzadeh (iD http://orcid.org/0000-0002-9398-9211

Sima Kheradmand Kia (D) https://orcid.org/0000-0002-2693-1853

Seti Boroomand (D) http://orcid.org/0000-0002-6319-598X

Mehdi Hedayati (D) https://orcid.org/0000-0001-5816-775X

\section{REFERENCES}

Ahmad, E., Fatima, M. T., Hoque, M., Owais, M., \& Saleemuddin, M. (2015). Fibrin matrices: The versatile therapeutic delivery systems. International Journal of Biological Macromolecules, 81, 121-136. https://doi.org/10.1016/j.ijbiomac.2015.07.054

Armitage, W. J. (1987). Cryopreservation of animal cells. Symposia of the Society for Experimental Biology, 41, 379-393.

Azambuja, R. M., Kraemer, D. C., \& Westhusin, M. E. (1998). Effect of low temperatures on in-vitro matured bovine oocytes. Theriogenology, 49(6), 1155-1164. https://doi.org/10.1016/S0093-691X(98)00063-6

Baiocchini, A., Montaldo, C., Conigliaro, A., Grimaldi, A., Correani, V., Mura, F., Ciccosanti, F., Rotiroti, N., Brenna, A., Montalbano, M., D'Offizi, G., Capobianchi, M. R., Alessandro, R., Piacentini, M., Schininà, M. E., Maras, B., Del Nonno, F., Tripodi, M., \& Mancone, C. (2016). Extracellular matrix molecular remodeling in human liver fibrosis evolution. PLoS One, 11(3), e0151736.

Bakhshpour, M., Idil, N., Perçin, I., \& Denizli, A. (2019). Biomedical applications of polymeric cryogels. Applied Sciences, 9(3), 553. https://doi.org/10.3390/app9030553

Barun, S. (2015). A review on applications \& advantages of cryopreservation in different fields of science. The Beats of Natural Sciences, 2, 1-6.

Baust, J. M., Van Buskirk, R., \& Baust, J. G. (2003). Normothermic, hypothermic and cryopreservation maintenance and storage of cells, tissues and organs in gel-based media. (U.S. Patent No. 6,632,666). U.S. Patent and Trademark Office.

Beattie, G. M., Crowe, J. H., Lopez, A. D., Cirulli, V., Ricordi, C., \& Hayek, A. (1997). Trehalose: A cryoprotectant that enhances recovery and preserves function of human pancreatic islets after long-term storage. Diabetes, 46(3), 519-523. https://doi.org/10.2337/diab.46.3.519

Ben-Ari, A., Rivkin, R., Frishman, M., Gaberman, E., Levdansky, L., \& Gorodetsky, R. (2009). Isolation and implantation of bone marrowderived mesenchymal stem cells with fibrin micro beads to repair a critical-size bone defect in mice. Tissue Engineering. Part A, 15(9), 2537-2546. https://doi.org/10.1089/ten.tea.2008.0567

Bissoyi, A., Nayak, B., Pramanik, K., \& Sarangi, S. K. (2014). Targeting cryopreservation-induced cell death: A review. Biopreservation and Biobanking, 12(1), 23-34. https://doi.org/10.1089/bio.2013.0032

von Bomhard, A., Elsässer, A., Ritschl, L. M., Schwarz, S., \& Rotter, N. (2016). Cryopreservation of endothelial cells in various cryoprotective agents 
and media-vitrification versus slow freezing methods. PLoS One, 11(2), e0149660. https://doi.org/10.1371/journal.pone.0149660

Brown, I. R. (1990). Induction of heat shock (stress) genes in the mammalian brain by hyperthermia and other traumatic events: $A$ current perspective. Journal of Neuroscience Research, 27(3), 247-255. https://doi.org/10.1002/jnr.490270302

Campos-Chillòn, L. F., Walker, D. J., De La Torre-Sanchez, J. F., \& Seidel, G. E. (2006). In vitro assessment of a direct transfer vitrification procedure for bovine embryos. Theriogenology, 65(6), 1200-1214. https://doi.org/10.1016/j.theriogenology.2005.07.015

Catoira, M. C., Fusaro, L., Di Francesco, D., Ramella, M., \& Boccafoschi, F. (2019). Overview of natural hydrogels for regenerative medicine applications. Journal of Materials Science: Materials in Medicine, 30(10), 1-10.

Chakrabarti, R., \& Schutt, C. E. (2001). The enhancement of PCR amplification by low molecular-weight sulfones. Gene, 274(1-2), 293-298. https://doi.org/10.1016/S0378-1119(01)00621-7

Chen, B., Wright, B., Sahoo, R., \& Connon, C. J. (2013). A novel alternative to cryopreservation for the short-term storage of stem cells for use in cell therapy using alginate encapsulation. Tissue Engineering Part $C$ : Methods, 19(7), 568-576.

Chen, K. L., Mylon, S. E., \& Elimelech, M. (2007). Enhanced aggregation of Alginate-coated iron oxide (Hematite) nanoparticles in the presence of calcium, strontium, and barium cations. Langmuir, 23(11), 5920-5928. https://doi.org/10.1021/la063744k

Chen, T., Acker, J. P., Eroglu, A., Cheley, S., Bayley, H., Fowler, A., \& Toner, M. (2001). Beneficial effect of intracellular trehalose on the membrane integrity of dried mammalian cells. Cryobiology, 43(2), 168-181. https://doi.org/10.1006/cryo.2001.2360

Choi, Y. H., \& Chang, Y. J. (2003). The influence of cooling rate, developmental stage, and the addition of sugar on the cryopreservation of larvae of the pearl oyster Pinctada fucata martensii. Cryobiology, 46(2), 190-193. https://doi.org/10.1016/S00112240(03)00016-6

Choudhery, M. S., Badowski, M., Muise, A., \& Harris, D. T. (2015). Effect of mild heat stress on the proliferative and differentiative ability of human mesenchymal stromal cells. Cytotherapy, 17(4), 359-368. https://doi.org/10.1016/j.jcyt.2014.11.003

Chung, H. J., \& Park, T. G. (2009). Injectable cellular aggregates prepared from biodegradable porous microspheres for adipose tissue engineering. Tissue Engineering. Part A, 15(6), 1391-1400. https://doi. org/10.1089/ten.tea.2008.0344

Cobo, F., Stacey, G. N., Hunt, C., Cabrera, C., Nieto, A., Montes, R., Cortés, J. L., Catalina, P., Barnie, A., \& Concha Á. (2005). Microbiological control in stem cell banks: Approaches to standardisation. Applied Microbiology and Biotechnology, 68(4), 456-466. https://doi.org/10.1007/s00253-005-0062-2

Coopman, K. (2013). Alternatives to cryopreservation for the short and long-term storage of mammalian cells. In: A. Colvert, \& H. Coty (Eds.), Cryopreservation: Technologies, applications and risks/outcomes (pp. 91-108). Nova Science Publishers.

Courtenay, E. S., Capp, M. W., Anderson, C. F., \& Record, M. T. (2000). Vapor pressure osmometry studies of osmolyte-protein interactions: Implications for the action of osmoprotectants in vivo and for the interpretation of 'osmotic stress' experiments in vitro. Biochemistry, 39(15), 4455-4471. https://doi.org/10.1021/ bi992887I

Criado, E., Albani, E., Novara, P. V., Smeraldi, A., Cesana, A., Parini, V., \& Levi-Setti, P. E. (2011). Human oocyte ultravitrification with a low concentration of cryoprotectants by ultrafast cooling: A new protocol. Fertility and Sterility, 95(3), 1101-1103. https://doi.org/ 10.1016/j.fertnstert.2010.11.015

Crowe, J. H. (2005). Stabilization of dry mammalian cells: Lessons from nature. Integrative and Comparative Biology, 45(5), 810-820. https:// doi.org/10.1093/icb/45.5.810
Crowe, J. H., Tablin, F., Wolkers, W. F., Gousset, K., Tsvetkova, N. M., \& Ricker, J. (2003). Stabilization of membranes in human platelets freezedried with trehalose. Chemistry and Physics of Lipids, 122(1-2), 41-52. https://doi.org/10.1016/S0009-3084(02)00177-9

Daei-farshbaf, N., Ardeshirylajimi, A., Seyedjafari, E., Piryaei, A., Fathabady, F. F., Hedayati, M., Salehi, M., Soleimani, M., Nazarian, H., Moradi, S.-L., \& Norouzian, M. (2014). BioceramicCollagen scaffolds loaded with human Adipose-tissue derived stem cells for bone tissue engineering. Molecular Biology Reports, 41(2), 741-749. https://doi.org/10.1007/s11033-013-2913-8

De Vries, E. G. E., Vellenga, E., Kluin-Nelemans, J. C., \& Mulder, N. H. (2004). The happy destiny of frozen haematopoietic stem cells: From immature stem cells to mature applications. European Journal of Cancer, 40(13), 1987-1992. https://doi.org/10.1016/j.ejca.2003. 11.040

Di, G., Wang, J., Liu, M., Wu, C. T., Han, Y., \& Duan, H. (2012). Development and evaluation of a trehalose-contained solution formula to preserve HUC-MSCs at $4^{\circ} \mathrm{C}$. Journal of Cellular Physiology, 227(3), 879-884. https://doi.org/10.1002/jcp.23066

Ebertz, S. L., \& McGann, L. E. (2004). Cryoinjury in endothelial cell monolayers. Cryobiology, 49(1), 37-44. https://doi.org/10.1016/j. cryobiol.2004.04.003

Ehrenreich, M., \& Ruszczak, Z. (2006). Update on tissue-engineered biological dressings. Tissue Engineering, 12(9), 2407-2424. https:// doi.org/10.1089/ten.2006.12.2407

El Assal, R., Abou-Elkacem, L., Tocchio, A., Pasley, S., Matosevic, S., Kaplan, D. L., Zylberberg, C., \& Demirci, U. (2019). Bioinspired preservation of natural killer cells for cancer immunotherapy. Advanced Science, 6(6), 1802045. https://doi.org/10.1002/advs. 201802045

El Assal, R., Guven, S., Gurkan, U. A., Gozen, I., Shafiee, H., Dalbeyler, S., Abdalla, N., Thomas, G., Fuld, W., Illigens, B. M. W., Estanislau, J., Khoory, J., Kaufman, R., Zylberberg, C., Lindeman, N., Wen, Q., Ghiran, I., \& Demirci, U. (2014). Bio-inspired cryo-ink preserves red blood cell phenotype and function during nanoliter vitrification. Advanced Materials, 26(33), 5815-5822. https://doi.org/10.1002/ adma.201400941

Erdag, G., Eroglu, A., Morgan, J. R., \& Toner, M. (2002). Cryopreservation of fetal skin is improved by extracellular trehalose. Cryobiology, 44(3), 218-228. https://doi.org/10.1016/S0011-2240(02)00023-8

Eroglu, A., Toner, M., \& Toth, T. L. (2002). Beneficial effect of microinjected trehalose on the cryosurvival of human oocytes. Fertility and Sterility, 77(1), 152-158. https://doi.org/10.1016/ S0015-0282(01)02959-4

Eroglu, A., Russo, M. J., Bieganski, R., Fowler, A., Cheley, S., Bayley, H., \& Toner, M. (2000). Intracellular trehalose improves the survival of cryopreserved mammalian cells. Nature Biotechnology, 18(2), 163-67. https://doi.org/10.1038/72608

Esfandiarei, M., Boroomand, S., Suarez, A., Si, X., Rahmani, M., \& McManus, B. (2007). Coxsackievirus B3 activates nuclear factor kappa B transcription factor via a Phosphatidylinositol-3 kinase/ protein kinase $b$-dependent pathway to improve host cell viability. Cellular Microbiology, 9(10), 2358-2371. https://doi.org/10.1111/j. 1462-5822.2007.00964.x

Fan, W.-X., Ma, X. H., Ge, D., Liu, T. Q., \& Cui, Z.-F. (2009). Cryoprotectants for the vitrification of corneal endothelial cells. Cryobiology, 58(1), 28-36. https://doi.org/10.1016/j.cryobiol.2008. 10.124

Gao, D., \& Critser, J. K. (2000). Mechanisms of cryoinjury in living cells. ILAR Journal, 41(4), 187-196. https://doi.org/10.1093/ilar.41.4.187

Gorodetsky, R., Levdansky, L., Gaberman, E., Gurevitch, O., Lubzens, E., \& McBride, W. H. (2011). Fibrin microbeads loaded with mesenchymal cells support their long-term survival while sealed at room temperature. Tissue Engineering Part C: Methods, 17(7), 745-755. https://doi.org/10.1089/ten.tec.2010.0644 
Gorodetsky, R., An, J., Levdansky, L., Vexler, A., Berman, E., Clark, R. A. F., Gailit, J., \& Marx, G. (1999). Fibrin microbeads (FMB) as biodegradable carriers for culturing cells and for accelerating wound Healing. Journal of Investigative Dermatology, 112(6), 866-72. https://doi.org/10.1046/j.1523-1747.1999.00600.x

Grein, T. A., Freimark, D., Weber, C., Hudel, K., Wallrapp, C., \& Czermak, P. (2010). Alternatives to dimethylsulfoxide for serum-free cryopreservation of human mesenchymal stem cells. The International Journal of Artificial Organs, 33(6), 370-380. https://doi.org/10.1177/ 039139881003300605

Hanna, J., \& Hubel, A. (2009). Preservation of stem cells. Organogenesis, 5(3), 134-137. https://doi.org/10.4161/org.5.3.9585

Hara, J., Tottori, J., Anders, M., Dadhwal, S., Asuri, P., \& Mobed-Miremadi, M. (2017). Trehalose effectiveness as a cryoprotectant in 2D and 3D cell cultures of human embryonic kidney cells. Artificial Cells, Nanomedicine, and Biotechnology, 45(3), 609-616. https://doi.org/ 10.3109/21691401.2016.1167698

He, X., Park, E. Y. H., Fowler, A., Yarmush, M. L., \& Toner, M. (2008). Vitrification by ultra-fast cooling at a low concentration of cryoprotectants in a quartz micro-capillary: A study using murine embryonic stem cells. Cryobiology, 56(3), 223-232. https://doi.org/ 10.1016/j.cryobiol.2008.03.005

Hegner, B., Weber, M., Dragun, D., \& Schulze-Lohoff, E. (2005). Differential regulation of smooth muscle markers in human bone marrow-derived mesenchymal stem cells. Journal of Hypertension, 23(6), 1191-1202. https://doi.org/10.1097/01.hjh.0000170382. $31085.5 d$

Heng, B. C., Ye, C. P., Liu, H., Toh, W. S., Rufaihah, A. J., Yang, Z., Bay, B. H., Ge, Z., Ouyang, H. W., Lee, E. H., \& Cao, T. (2006). Loss of viability during freeze-thaw of intact and adherent human embryonic stem cells with conventional slow-cooling protocols is predominantly due to apoptosis rather than cellular necrosis. Journal of Biomedical Science, 13(3), 433-445. https://doi.org/10.1007/ s11373-005-9051-9

Heo, Y. S., Nagrath, S., Moore, A. L., Zeinali, M., Irimia, D., Stott, S. L., Toth, T. L., \& Toner, M. (2015). Universal' vitrification of cells by ultra-fast cooling. Technology, 3(1), 64-71. https://doi.org/10.1142/ S2339547815500053

Hunt, C. J. (2007). The banking and cryopreservation of human embryonic stem cells. Transfusion Medicine and Hemotherapy, 34(4), 293-304. https://doi.org/10.1159/000104458

Jain, M., Rajan, R., Hyon, S. H., \& Matsumura, K. (2014). Hydrogelation of Dextran-based polyampholytes with cryoprotective properties via click chemistry. Biomaterials Science, 2(3), 308-317. https://doi.org/ 10.1039/C3BM60261C

Jang, T. H., Park, S. C., Yang, J. H., Kim, J. Y., Seok, J. H., Park, U. S., Choi, C. W., Lee, S. R., \& Han, J. (2017). Cryopreservation and its clinical applications. Integrative Medicine Research, 6(1), 12-18. https://doi.org/10.1016/j.imr.2016.12.001

Janz, F. L., Debes, A. A., Cavaglieri, R. C., Duarte, S. A., Romão, C. M., Morón, A. F., Zugaib, M., \& Bydlowski, S. P. (2012). Evaluation of distinct freezing methods and cryoprotectants for human amniotic fluid stem cells cryopreservation. Journal of Biomedicine and Biotechnology, 2012, 1-10. https://doi.org/10.1155/2012/649353

Jenkins, E. C., Ye, L., \& Silverman, W. P. (2012). Does the cryogenic freezing process cause shorter telomeres? Cryobiology, 65(1), 72-73. https://doi.org/10.1016/j.cryobiol.2012.03.005

Ji, L., de Pablo, J. J., \& Palecek, S. P. (2004). Cryopreservation of adherent human embryonic stem cells. Biotechnology and Bioengineering, 88(3), 299-312. https://doi.org/10.1002/bit.20243

Jin, B., Kusanagi, K., Ueda, M., Valdez, D. M., Seki, S., Edashige, K., \& Kasai, M. (2008). Formation of extracellular and intracellular ice during warming of vitrified mouse morulae and its effect on embryo survival. Cryobiology, 56(3), 233-240. https://doi.org/10.1016/j. cryobiol.2008.03.004
Kang, S.-W., Seo, S.-W., Choi, C. Y., \& Kim, B.-S. (2008). Porous poly (Lactic-Co-Glycolic acid) microsphere as cell culture substrate and cell transplantation vehicle for adipose tissue engineering. Tissue Engineering Part C: Methods, 14(1), 25-34. https://doi.org/10.1089/ tec. 2007.0290

Karlsson, J. O. M., \& Toner, M. (1996). Long-term storage of tissues by cryopreservation: Critical issues. Biomaterials, 17(3), 243-256. https://doi.org/10.1016/0142-9612(96)85562-1

Katenz, E., Vondran, F. W. R., Schwartlander, R., Pless, G., Gong, X., Cheng, X., Neuhaus, P., \& Sauer, I. M. (2007). Cryopreservation of primary human hepatocytes: The benefit of trehalose as an additional cryoprotective agent. Liver Transplantation, 13(1), 38-45. https://doi.org/10.1002/It.20921

Katsen-Globa, A., Meiser, I., Petrenko, Y. A., Ivanov, R. V., Lozinsky, V. I., Zimmermann, H., \& Petrenko, A. Y. (2014). Towards ready-to-use 3D scaffolds for regenerative medicine: Adhesion-based cryopreservation of human mesenchymal stem cells attached and spread within Alginate-Gelatin cryogel scaffolds. Journal of Materials Science. Materials in medicine, 25(3), 857-871. https://doi.org/10. 1007/s10856-013-5108-x

Kaushik, S., \& Kaur, J. (2005). Effect of chronic cold stress on intestinal epithelial cell proliferation and inflammation in rats. Stress, 8(3), 191-197. https://doi.org/10.1080/10253890500245953

Khan, R., \& Khan, M. (2013). Use of collagen as a biomaterial: An update. Journal of Indian Society of Periodontology, 17(4), 539-542. https:// doi.org/10.4103/0972-124X.118333

Kim, S. J., Park, J. H., Lee, J. E., Kim, J. M., Lee, J. B., Moon, S. Y., Roh, S. I., Kim, C. G., \& Yoon, H. S. (2004). Effects of type IV collagen and laminin on the cryopreservation of human embryonic stem cells. Stem Cells, 22(6), 950-961. https://doi.org/10.1634/stemcells.226-950

Kumari, J., \& Kumar, A. (2017). Development of polymer based cryogel matrix for transportation and storage of mammalian cells. Scientific Reports, 7, 41551. https://doi.org/10.1038/srep41551

Kuwayama, M., Vajta, G., Kato, O., \& Leibo, S. P. (2005). Highly efficient vitrification method for cryopreservation of human oocytes. Reproductive BioMedicine Online, 11(3), 300-308. https://doi.org/ 10.1016/S1472-6483(10)60837-1

Lawson, A., Ahmad, H., \& Sambanis, A. (2011). Cytotoxicity effects of cryoprotectants as single-component and cocktail vitrification solutions. Cryobiology, 62(2), 115-122. https://doi.org/10.1016/j. cryobiol.2011.01.012

Lee, C. H., Singla, A., \& Lee, Y. (2001). Biomedical applications of collagen. International Journal of Pharmaceutics, 221(1-2), 1-22. https://doi. org/10.1016/S0378-5173(01)00691-3

Lee, H. J., Elmoazzen, H., Wright, D. L., Biggers, J., Rueda, B. R., Heo, Y. S., Toner, M., \& Toth, T. L. (2009). Ultra-rapid vitrification of oocytes in low cryoprotectant concentration using quart capillary. Fertility and Sterility, 92(3), S71. https://doi.org/10.1016/j.fertnstert.2009.07.274

Lee, Y.-A., Kim, Y.-H., Kim, B.-J., Kim, B.-G., Kim, K.-J., Auh, J.-H., Schmidt, J. A., \& Ryu, B.-Y. (2013). Cryopreservation in trehalose preserves functional capacity of murine spermatogonial stem cells. PLoS One, 8(1), e54889. https://doi.org/10.1371/journal. pone.0054889

Li, A. P. (2007). Human hepatocytes: Isolation, cryopreservation and applications in drug development. Chemico-Biological Interactions, 168(1), 16-29. https://doi.org/10.1016/j.cbi.2007.01.001

Li, Y., Si, W., Zhang, X., Dinnyes, A., \& Ji, W. (2003). Effect of amino acids on cryopreservation of cynomolgus monkey (Macaca Fascicularis) sperm. American Journal of Primatology, 59(4), 159-165. https://doi. org/10.1002/ajp.10073

Linnebacher, M., Maletzki, C., Ostwald, C., Klier, U., Krohn, M., Klar, E., \& Prall, F. (2010). Cryopreservation of human colorectal carcinomas prior to xenografting. BMC Cancer, 10(1), 362. https://doi.org/10. 1186/1471-2407-10-362 
Liu, M., Zhang, X., Guo, H., Zhu, Y., Wen, C., Sui, X., Yang, J., \& Zhang, L. (2019). Dimethyl sulfoxide-free cryopreservation of chondrocytes based on zwitterionic molecule and polymers. Biomacromolecules, 20(10), 3980-3988.

Malpique, R., Osório, L. M., Ferreira, D. S., Ehrhart, F., Brito, C., Zimmermann, H., \& Alves, P. M. (2010). Alginate encapsulation as a novel strategy for the cryopreservation of neurospheres. Tissue Engineering Part C: Methods, 16(5), 965-977. https://doi.org/10. 1089/ten.tec.2009.0660

Martinetti, D., Colarossi, C., Buccheri, S., Denti, G., Memeo, L., \& Vicari, L. (2017). Effect of trehalose on cryopreservation of pure peripheral blood stem cells. Biomedical Reports, 6(3), 314-318. https://doi.org/ 10.3892/br.2017.859

McGann, L. E. (1978). Differing actions of penetrating and nonpenetrating cryoprotective agents. Cryobiology, 15(4), 382-390. https://doi.org/ 10.1016/0011-2240(78)90056-1

Meryman, H. T. (2007). Cryopreservation of living cells: Principles and practice. Transfusion, 47(5), 935-945. https://doi.org/10.1111/j. 1537-2995.2007.01212.x

Miyamoto, Y., Enosawa, S., Takeuchi, T., \& Takezawa, T. (2009). Cryopreservation in situ of cell monolayers on collagen vitrigel membrane culture substrata: Ready-to-Use preparation of primary hepatocytes and ES cells. Cell Transplantation, 18(5-6), 619-626. https://doi.org/10.1177/096368970901805-618

Miyamoto, Y., Ikeuchi, M., Noguchi, H., \& Hayashi, S. (2018). Long-term cryopreservation of human and other mammalian cells at $-80^{\circ} \mathrm{C}$ for 8 years. Cell Medicine, 10, 2155179017733148. https://doi.org/10. $1177 / 2155179017733148$

Mørch, Ý. A., Donati, I., Strand, B. L., \& Skjåk-Bræk, G. (2006). Effect of $\mathrm{Ca} 2+, \mathrm{Ba} 2+$, and $\mathrm{Sr} 2+$ on alginate microbeads. Biomacromolecules, 7(5), 1471-1480. https://doi.org/10.1021/bm060010d

Morris, G. J., Goodrich, M., Acton, E., \& Fonseca, F. (2006). The high viscosity encountered during freezing in glycerol solutions: Effects on cryopreservation. Cryobiology, 52(3), 323-334. https://doi.org/ 10.1016/j.cryobiol.2006.01.003

Motta, J. P. R., Paraguassú-Braga, F. H., Bouzas, L. F., \& Porto, L. C. (2014). Evaluation of intracellular and extracellular trehalose as a cryoprotectant of stem cells obtained from umbilical cord blood. Cryobiology, 68(3), 343-348. https://doi.org/10.1016/j.cryobiol. 2014.04.007

Moussa, M., Dumont, F., Perrier-Cornet, J.-M., \& Gervais, P. (2008). Cell inactivation and membrane damage after long-term treatments at sub-zero temperature in the supercooled and frozen states. Biotechnology and Bioengineering, 101(6), 1245-1255. https://doi. org/10.1002/bit.21981

Nema, R., \& Khare, S. (2012). An animal cell culture: Advance technology for modern research. Advances in Bioscience and Biotechnology, 3(3), 219-226. https://doi.org/10.4236/abb.2012.33030

Nienow, A. W., Hewitt, C. J., Heathman, T. R. J., Glyn, V. A. M., Fonte, G. N., Hanga, M. P., Coopman, K., \& Rafiq, Q. A. (2016). Agitation conditions for the culture and detachment of HMSCs from microcarriers in multiple bioreactor platforms. Biochemical Engineering Journal, 108, 24-29. https://doi.org/10.1016/j.bej.2015.08.003

Nomura, M., Muramoto, Y., Yasuda, S., Takabe, T., \& Kishitani, S. (1995). The accumulation of glycinebetaine during cold acclimation in early and late cultivars of barley. Euphytica, 83(3), 247-250. https://doi. org/10.1007/BF01678137

Park, J. S., Yang, H. N., Woo, D. G., Jeon, S. Y., \& Park, K.-H. (2011). The promotion of chondrogenesis, osteogenesis, and adipogenesis of human mesenchymal stem cells by multiple growth factors incorporated into nanosphere-coated microspheres. Biomaterials, 32(1), 28-38. https://doi.org/10.1016/j.biomaterials.2010.08.088

Pi, C.-H., Yu, G., Petersen, A., \& Hubel, A. (2018). Characterizing the 'sweet spot' for the preservation of a T-cell line using osmolytes. Scientific Reports, 8(1), 1-13.
Pi, C.-H., Yu, G., Dosa, P. I., \& Hubel, A. (2019). Characterizing modes of action and interaction for multicomponent osmolyte solutions on Jurkat cells. Biotechnology and Bioengineering, 116(3), 631-643. https://doi.org/10.1002/bit.26880

Pi, C.-H., Hornberger, K., Dosa, P., \& Hubel, A. (2020). Understanding the freezing responses of $T$ cells and other subsets of human peripheral blood mononuclear cells using DSMO-Free cryoprotectants. Cytotherapy, 22(5), 291-300.

Place, E. S., Rojo, L., Gentleman, E., Sardinha, J. P., \& Stevens, M. M. (2011). Strontium-and zinc-alginate hydrogels for bone tissue engineering. Tissue Engineering. Part A, 17(21-22), 2713-2722. https://doi.org/10.1089/ten.tea.2011.0059

Pu, L., Cui, X., Fink, B., Cibull, M., \& Gao, D. (2005). Cryopreservation of adipose tissues: The role of trehalose. Aesthetic Surgery Journal, 25(2), 126-131. https://doi.org/10.1016/j.asj.2005.01.003

Rajangam, T., \& An, S. S. A. (2013). Fibrinogen and fibrin based micro and nano scaffolds incorporated with drugs, proteins, cells and genes for therapeutic biomedical applications. International Journal of Nanomedicine, 8, 3641.

Ranucci, C. S., Kumar, A., Batra, S. P., \& Moghe, P. V. (2000). Control of hepatocyte function on collagen foams: Sizing matrix pores toward selective induction of 2-D and 3-D cellular morphogenesis. Biomaterials, 21(8), 783-793. https://doi.org/10.1016/S0142-9612(99)00238-0

Reddig, P. J., \& Juliano, R. L. (2005). Clinging to life: Cell to matrix adhesion and cell survival. Cancer and Metastasis Reviews, 24(3), 425-439. https://doi.org/10.1007/s10555-005-5134-3

Rubinstein, P., Dobrila, L., Rosenfield, R. E., Adamson, J. W., Migliaccio, G., Migliaccio, A. R., Taylor, P. E., \& Stevens, C. E. (1995). Processing and cryopreservation of Placental/Umbilical cord blood for unrelated bone marrow reconstitution. Proceedings of the National Academy of Sciences of the United States of America, 92(22), 10119-10122. https://doi.org/10.1073/pnas.92.22.10119

Rudolph, A. S., \& Crowe, J. H. (1985). Membrane stabilization during freezing: The role of two natural cryoprotectants, trehalose and proline. Cryobiology, 22(4), 367-377. https://doi.org/10.1016/ 0011-2240(85)90184-1

Ruoß, M., Häussling, V., Schügner, F., Damink, L. O., Lee, S., Ge, L., Ehnert, S., \& Nussler, A. (2018). A standardized collagen-based scaffold improves human hepatocyte shipment and allows metabolic studies over 10 days. Bioengineering, 5(4), 86. https://doi.org/10. 3390/bioengineering5040086

Stewart, S., \& He, X. (2018). Intracellular delivery of trehalose for cell banking. Langmuir, 35(23), 7414-7422.

Sarkar, J., \& Kumar, A. (2016). Thermo-responsive polymer aided spheroid culture in cryogel based platform for high throughput drug screening. The Analyst, 141(8), 2553-2567. https://doi.org/10. 1039/C6AN00356G

Satpathy, G. R., Török, Z., Bali, R., Dwyre, D. M., Little, E., Walker, N. J., Tablin, F., Crowe, J. H., \& Tsvetkova, N. M. (2004). Loading red blood cells with trehalose: A step towards biostabilization. Cryobiology, 49(2), 123-136. https://doi.org/10.1016/j.cryobiol.2004.06.001

Scheinkönig, C., Kappicht, S., Kolb, H.-J., \& Schleuning, M. (2004). Adoption of long-term cultures to evaluate the cryoprotective potential of trehalose for freezing hematopoietic stem cells. Bone Marrow Transplantation, 34(6), 531-536. https://doi.org/10.1038/sj. bmt.1704631

Schoenhard, J. A., \& Hatzopoulos, A. K. (2010). Stem cell therapy: Pieces of the puzzle. Journal of Cardiovascular Translational Research, 3(1), 49-60. https://doi.org/10.1007/s12265-009-9148-z

Schoof, H., Apel, J., Heschel, I., \& Rau, G. (2001). Control of pore structure and size in freeze-dried collagen sponges. Journal of Biomedical Materials Research, 58(4), 352-357. https://doi.org/10.1002/jbm.1028

Scott, K. L., Lecak, J., \& Acker, J. P. (2005). Biopreservation of red blood cells: Past, present, and future. Transfusion Medicine Reviews, 19(2), 127-142. https://doi.org/10.1016/j.tmrv.2004.11.004 
Seo, J. M., Sohn, M. Y., Suh, J. S., Atala, A., Yoo, J. J., \& Shon, Y.-H. (2011). Cryopreservation of amniotic fluid-derived stem cells using natural cryoprotectants and low concentrations of dimethylsulfoxide. Cryobiology, 62(3), 167-173. https://doi.org/10.1016/j.cryobiol. 2011.02.003

Serra, M., Cláudia, C., Malpique, R., Brito, C., Jensen, J., Bjorquist, P., Carrondo, M. J. T., \& Alves, P. M. (2011). Microencapsulation technology: A powerful tool for integrating expansion and cryopreservation of human embryonic stem cells. PLoS One, 6(8), e23212.

Shanina, I. V., Kravchenko, L. P., Fuller, B. J., \& Grischenko, V. I. (2000). A comparison of a sucrose-based solution with other preservation media for cold storage of isolated hepatocytes. Cryobiology, 41(4), 315-318. https://doi.org/10.1006/cryo.2000.2286

Shao, Q., \& Jiang, S. (2015). Molecular understanding and design of zwitterionic materials. Advanced Materials, 27(1), 15-26. https://doi. org/10.1002/adma.201404059

Sharma, S. D. (2005). Cryopreservation of somatic embryos-An overview. Biotechnology, 4(1), 47-55.

Shimony, N., Gorodetsky, R., Marx, G., Gal, D., Rivkin, R., Ben-Ari, A., Landsman, A., \& Haviv, Y. S. (2006). Fibrin microbeads (FMB) as a 3D platform for kidney gene and cell therapy. Kidney International, 69(3), 625-633. https://doi.org/10.1038/sj.ki.5000099

Stacey, G. N., \& Masters, J. R. (2008). Cryopreservation and banking of mammalian cell lines. Nature Protocols, 3(12), 1981-1989. https:// doi.org/10.1038/nprot.2008.190

Stacey, G. N., Connon, C. J., Coopman, K., Dickson, A. J., Fuller, B., Hunt, C. J., Kemp, P., Kerby, J., Man, J., Matejtschuk, P., Moore, H., Morris, J., Oreffo, R. O., Slater, N., Ward, S., Wiggins, C., \& Zimmermann, H. (2017). Preservation and stability of cell therapy products: Recommendations from an expert workshop. Regenerative Medicine, 12(5), 553-564. https://doi.org/10.2217/rme-2017-0073

Stefansson, S., Han, S., Jeon, Y. I., Chung, D. S., Hwang, P., Le, H., Warden, J. L., \& Ho, D. (2017). Transporting mammalian cells at ambient temperature: A viable alternative to dry ice. Advances in Bioscience and Biotechnology, 8(4), 127-133. https://doi.org/10. 4236/abb.2017.84009

Stevens, V. L., Patel, A. V., Feigelson, H. S., Rodriguez, C., Thun, M. J., \& Calle, E. E. (2007). Cryopreservation of whole blood samples collected in the field for a large epidemiologic study. Cancer Epidemiology, Biomarkers \& Prevention, 16(10), 2160-2163. https:// doi.org/10.1158/1055-9965.EPI-07-0604

Sundaramurthi, P., \& Suryanarayanan, R. (2012). Calorimetry and complementary techniques to characterize frozen and freeze-dried systems. Advanced Drug Delivery Reviews, 64(5), 384-395. https:// doi.org/10.1016/j.addr.2011.12.004

Suuronen, E. J., Kuraitis, D., \& Ruel, M. (2008). Improving cell engraftment with tissue engineering. Seminars in Thoracic and Cardiovascular Surgery, 20(2), 110-114. https://doi.org/10.1053/j.semtcvs.2008. 03.005

Svalgaard, J. D., Talkhoncheh, M. S., Haastrup, E. K., Munthe-Fog, L., Clausen, C., Hansen, M. B., Andersen, P., Gørløv, J. S., Larsson, J., \& Fischer-Nielsen, A. (2018). Pentaisomaltose, an alternative to DMSO. Engraftment of cryopreserved human CD34+ cells in immunodeficient NSG mice. Cell Transplantation, 27(9), 1407-1412. https:// doi.org/10.1177/0963689718786226

Swioklo, S., Constantinescu, A., \& Connon, C. J. (2016). Alginateencapsulation for the improved hypothermic preservation of human adipose-derived stem cells. Stem Cells Translational Medicine, 5(3), 339-349. https://doi.org/10.5966/sctm.2015-0131

Syme, R., Bewick, M., Stewart, D., Porter, K., Chadderton, T., \& Glück, S. (2004). The role of depletion of dimethyl sulfoxide before autografting: On hematologic recovery, side effects, and toxicity. Biology of Blood and Marrow Transplantation, 10(2), 135-141. https://doi.org/10.1016/j.bbmt.2003.09.016
Theus, M. H., Wei, L., Cui, L., Francis, K., Hu, X., Keogh, C., \& Yu, S. P. (2008). In vitro hypoxic preconditioning of embryonic stem cells as a strategy of promoting cell survival and functional benefits after transplantation into the ischemic rat brain. Experimental Neurology, 210(2), 656-670. https://doi.org/10.1016/j.expneurol.2007.12.020

Tounkara, F. K., Dumont, N., Fournier, S., Boyer, L., Nadeau, P., \& Pineault, N. (2012). Mild hyperthermia promotes and accelerates development and maturation of erythroid cells. Stem Cells and Development, 21(17), 3197-3208. https://doi.org/10.1089/scd. 2012.0112

Troitzsch, R. Z., Vass, H., Hossack, W. J., Martyna, G. J., \& Crain, J. (2008). Molecular mechanisms of cryoprotection in aqueous proline: Light scattering and molecular dynamics simulations. The Journal of Physical Chemistry B, 112(14), 4290-4297. https://doi.org/10. 1021/jp076713m

Trusal, L. R., Guzman, A. W., \& Baker, C. J. (1984). Characterization of freeze-thaw induced ultrastructural damage to endothelial cells in vitro. In Vitro, 20(4), 353-364. https://doi.org/10.1007/ BF02618599

Umemura, E., Yamada, Y., Nakamura, S., Ito, K., Hara, K., \& Ueda, M. (2011). Viable cryopreserving tissue-engineered cell-biomaterial for cell banking therapy in an effective cryoprotectant. Tissue Engineering Part C: Methods, 17(8), 799-807. https://doi.org/10. 1089/ten.tec.2011.0003

Vajta, G., Holm, P., Kuwayama, M., Booth, P. J., Jacobsen, H., Greve, T., \& Callesen, H. (1998). Open pulled straw (OPS) vitrification: A new way to reduce cryoinjuries of bovine ova and embryos. Molecular Reproduction and Development, 51(1), 53-58. https://doi.org/10. 1002/(SICI)1098-2795(199809)51:1<53::AID-MRD6>3.0.CO;2-V

Vergara, M., Becerra, S., Berrios, J., Osses, N., Reyes, J., Rodriguez-Moya, M., Gonzalez, R., \& Altamirano, C. (2014). Differential effect of culture temperature and specific growth rate on $\mathrm{CHO}$ cell behavior in chemostat culture. PLoS One, 9(4), e93865.

Vigneault, C., Thompson, J., Wu, S., Hui, K. P. C., \& LeBlanc, D. I. (2009). Transportation of fresh horticultural produce. Postharvest Technologies for Horticultural Crops, 2(1), 1-24.

Vrana, N. E., Matsumura, K., Hyon, S. H., Geever, L. M., Kennedy, J. E., Lyons, J. G., Higginbotham, C. L., Cahill, P. A., \& McGuinness, G. B. (2012). Cell encapsulation and cryostorage in PVA-Gelatin cryogels: Incorporation of carboxylated E-poly-L-lysine as cryoprotectant. Journal of Tissue Engineering and Regenerative Medicine, 6(4), 280-290. https://doi.org/10.1002/term.431

Wang, J., Chen, P., Xu, J., Zou, J. X., Wang, H., \& Chen, H. W. (2015). Transporting cells in semi-solid gel condition and at ambient temperature. PLoS One, 10(6), e0128229. https://doi.org/10.1371/ journal.pone.0128229

Wang, J., Wei, Y., Zhao, S., Zhou, Y., He, W., Zhang, Y., \& Deng, W. (2017). The analysis of viability for mammalian cells treated at different temperatures and its application in cell shipment. PLoS One, 12(4), e0176120. https://doi.org/10.1371/journal.pone.0176120

Watanabe, K., Imanishi, S., Akiduki, G., Cornette, R., \& Okuda, T. (2016). Air-dried cells from the anhydrobiotic insect, Polypedilum vanderplanki, can survive long term preservation at room temperature and retain proliferation potential after rehydration. Cryobiology, 73(1), 93-98. https://doi.org/10.1016/j.cryobiol.2016.05.006

Wheatley, S. P., \& Wheatley, D. N. (2019). Transporting cells over several days without dry-ice. Journal of Cell Science, 132(21), jcs238139. https://doi.org/10.1242/jcs.238139

Wille, J. J., Burdge, J. J., \& Park, J. Y. (2014). Methods for the preparation of an autologous serum-free cultured epidermis and for autografting applications, Epidermal cells (pp. 203-218). Springer.

Wise, H., Abel, P. W., \& Cawkill, D. (2009). Use of reduced temperature cell pausing to enhance flexibility of cell-based assays. SLAS Discovery, 14(6), 716-722. https://doi.org/10.1177/1087057109335748 
Wolkers, W. F., Tablin, F., \& Crowe, J. H. (2002). From anhydrobiosis to freeze-drying of eukaryotic cells. Comparative Biochemistry and Physiology. Part A, Molecular \& Integrative Physiology, 131(3), 535-543. https://doi.org/10.1016/s1095-6433(01)00505-0

Wolkers, W. F., Walker, N. J., Tablin, F., \& Crowe, J. H. (2001). Human platelets loaded with trehalose survive freeze-drying. Cryobiology, 42(2), 79-87. https://doi.org/10.1006/cryo.2001.2306

Woods, E. J., Pollok, K. E., Byers, M. A., Perry, B. C., Purtteman, J., Heimfeld, S., \& Gao, D. (2007). Cord blood stem cell cryopreservation. Transfusion Medicine and Hemotherapy, 34(4), 276-285. https://doi.org/10.1159/000104183

Wright, B., Cave, R. A., Cook, J. P., Khutoryanskiy, V. V., Mi, S., Chen, B., Leyland, M., \& Connon, C. J. (2012). Enhanced viability of corneal epithelial cells for efficient transport/storage using a structurally modified calcium alginate hydrogel. Regenerative Medicine, 7(3), 295-307. https://doi.org/10.2217/rme.12.7

Yang, J., Cai, N., Zhai, H., Zhang, J., Zhu, Y., \& Zhang, L. (2016). Natural zwitterionic betaine enables cells to survive ultrarapid cryopreservation. Scientific Reports, 6, 37458. https://doi.org/10.1038/ srep37458

Yang, J., Pan, C., Sui, X., Cai, N., Zhang, J., Zhu, Y., \& Zhang, L. (2017). The hypothermic preservation of mammalian cells with assembling extracellular-matrix-mimetic microparticles. Journal of Materials Chemistry B, 5(8), 1535-1541. https://doi.org/10.1039/ C6TB03206K

Yang, J., Sui, X., Li, Q., Zhao, W., Zhang, J., Zhu, Y., Chen, P., \& Zhang, L. (2019). In situ encapsulation of postcryopreserved cells using alginate polymer and zwitterionic betaine. ACS Biomaterials Science \& Engineering, 5(5), 2621-2630. https://doi.org/10.1021/acsbiomaterials.9b00249

Yang, L., Li, C., Chen, L., \& Li, Z. (2009). An Agarose-gel based method for transporting cell lines. Current Chemical Genomics, 3, 50-53. https:// doi.org/10.2174/1875397300903010050

Yao, T., \& Asayama, Y. (2017). Animal-cell culture media: History, characteristics, and current issues. Reproductive Medicine and Biology, 16(2), 99-117. https://doi.org/10.1002/rmb2.12024

Yokomise, H., Inui, K., Wada, H., Hasegawa, S., Ohno, N., \& Hitomi, S. (1995). Reliable cryopreservation of trachea for one month in a new trehalose solution. The Journal of Thoracic and Cardiovascular Surgery, 110(2), 382-385. https://doi.org/10.1016/S0022-5223(95)70234-2

Yong, K. W., Safwani, W. K. Z. W., Xu, F., Zhang, X., Choi, J. R., Abas, W. A. B. W., Omar, S. Z., Azmi, M. A. N., Chua, K. H., \& Pingguan-Murphy, B. (2017). Assessment of tumourigenic potential in long-term cryopreserved human adipose-derived stem cells. Journal of Tissue Engineering and Regenerative Medicine, 11(8), 2217-2226. https://doi.org/10.1002/term.2120

Yu, G., Li, R., \& Hubel, A. (2018). Interfacial interactions of sucrose during cryopreservation detected by Raman spectroscopy. Langmuir, 35(23), 7388-7395. https://doi.org/10.1021/acs.langmuir.8b01616

Zhang, W., \& He, X. (2011). Microencapsulating and banking living cells for cell-based medicine. Journal of Healthcare Engineering, 2(4), 427-446. https://doi.org/10.1260/2040-2295.2.4.427

Zhang, X. B., Li, K., Yau, K. H., Tsang, K. S., Fok, T. F., Li, C. K., Lee, S. M., \& Yuen, P. M. P. (2003). Trehalose ameliorates the cryopreservation of cord blood in a preclinical system and increases the recovery of CFUs, long-term culture-initiating cells, and nonobese Diabetic-SCID repopulating cells. Transfusion, 43(2), 265-272. https://doi.org/10. 1046/j.1537-2995.2003.00301.x

Zimmermann, H., Zimmermann, D., Reuss, R., Feilen, P. J., Manz, B., Katsen, A., Weber, M., Ihmig, F. R., Ehrhart, F., Gessner, P., Behringer, M., Steinbach, A., Wegner, L. H., Sukhorukov, V. L., Vásquez, J. A., Schneider, S., Weber, M. M., Volke, F., Wolf, R., \& Zimmermann, U. (2005). Towards a medically approved technology for Alginate-based microcapsules allowing long-term immunoisolated transplantation. Journal of Materials Science. Materials in Medicine, 16(6), 491-501. https://doi.org/10.1007/s10856-005-0523-2

How to cite this article: Heydarzadeh, S., Kheradmand Kia, S., Boroomand, S., \& Hedayati, M. (2022). Recent developments in cell shipping methods. Biotechnology and Bioengineering, 119, 2985-3006. https://doi.org/10.1002/bit.28197 